\title{
THE ENERGY-CRITICAL QUANTUM HARMONIC OSCILLATOR
}

\author{
CASEY JAO
}

\begin{abstract}
We consider the energy critical nonlinear Schrödinger equation in dimensions $d \geq 3$ with a harmonic oscillator potential $V(x)=\frac{1}{2}|x|^{2}$. When the nonlinearity is defocusing, we prove global wellposedness for all initial data in the energy space $\Sigma$, consisting of all functions $u_{0}$ such that both $\nabla u_{0}$ and $x u_{0}$ belong to $L^{2}$. This result extends a theorem of Killip-Visan-Zhang 23], which treats the radial case. For the focusing problem, we obtain global wellposedness for all data satisfying an analogue of the usual size restriction in terms of the ground state $W$. The proof uses the concentration compactness variant of the induction on energy paradigm. In particular, we develop a linear profile decomposition adapted to the propagator $\exp \left[i t\left(\frac{1}{2} \Delta-\frac{1}{2}|x|^{2}\right)\right]$ for bounded sequences in $\Sigma$.
\end{abstract}

\section{Contents}

1. Introduction

Acknowledgements

2. Preliminaries

2.1. Notation and basic estimates

2.2. Littlewood-Paley theory

2.3. Local smoothing

3. Local theory

4. Concentration compactness

4.1. An Inverse Strichartz Inequality

4.2. Convergence of linear propagators

4.3. End of Proof of Inverse Strichartz

4.4. Linear profile decomposition

5. The case of concentrated initial data

6. Palais-Smale and the proof of Theorem 1.2

7. Proof of Theorem 1.3

8. Bounded linear potentials

References

\section{INTRODUCTION}

We study the initial value problem for the energy-critical nonlinear Schrödinger equation on $\mathbf{R}^{d}, d \geq 3$, with a harmonic oscillator potential:

$$
\left\{\begin{array}{c}
i \partial_{t} u=\left(-\frac{1}{2} \Delta+\frac{1}{2}|x|^{2}\right) u+\mu|u|^{\frac{4}{d-2}} u, \quad \mu= \pm 1, \\
u(0)=u_{0} \in \Sigma\left(\mathbf{R}^{d}\right) . \\
1
\end{array}\right.
$$


The equation is defocusing if $\mu=1$ and focusing if $\mu=-1$. Solutions to this PDE conserve energy, which is defined as

$$
E(u(t))=\int_{\mathbf{R}^{d}}\left[\frac{1}{2}|\nabla u(t)|^{2}+\frac{1}{2}|x|^{2}|u(t)|^{2}+\frac{d-2}{d} \mu|u(t)|^{\frac{2 d}{d-2}}\right] d x=E(u(0)) .
$$

Indeed (1.1) can be viewed as defining the (formal) Hamiltonian flow of $E$. The term "energy-critical" refers to the fact that if we ignore the $|x|^{2} / 2$ term in the equation and the energy, the scaling

$$
u(t, x) \mapsto u^{\lambda}(t, x):=\lambda^{-\frac{2}{d-2}} u\left(\lambda^{-2} t, \lambda^{-1} x\right)
$$

preserves both the equation and the energy. We take our initial data in the weighted Sobolev space $\Sigma$, which is the natural space of functions associated with the energy functional. This space is equipped with the norm

$$
\|f\|_{\Sigma}^{2}=\|\nabla f\|_{L^{2}}^{2}+\|x f\|_{L^{2}}^{2}=\|f\|_{\dot{H}^{1}}^{2}+\|f\|_{L^{2}\left(|x|^{2} d x\right)}^{2}
$$

We will frequently employ the notation

$$
H=-\frac{1}{2} \Delta+\frac{1}{2}|x|^{2}, \quad F(z)=\mu|z|^{\frac{4}{d-2}} z .
$$

Let us first clarify what we mean by a solution.

Definition. A (strong) solution to (1.1) is a function $u: I \times \mathbf{R}^{d} \rightarrow \mathbf{C}$ that belongs to $C_{t}^{0}(K ; \Sigma)$ for every compact interval $K \subset I$, and that satisfies the Duhamel formula

$$
u(t)=e^{-i t H} u(0)-i \int_{0}^{t} e^{-i(t-s) H} F(u(s)) d s \text { for all } t \in I .
$$

The hypothesis on $u$ implies that $F(u) \in C_{t, l o c}^{0} L_{x}^{\frac{2 d}{d+2}}\left(I \times \mathbf{R}^{d}\right)$. Consequently, the right side above is well-defined, at least as a weak integral of tempered distributions.

Equation (1.1) and its variants

$$
i \partial_{t} u=\left(-\frac{1}{2} \Delta+V\right) u+F(u), \quad V= \pm \frac{1}{2}|x|^{2}, \quad F(u)= \pm|u|^{p} u, \quad p>0
$$

have received considerable attention, especially in the energy-subcritical regime $p<4 /(d-2)$. The equation with a confining potential $V=|x|^{2} / 2$ has been used to model Bose-Einstein condensates in a trap (see [33], for example). Let us briefly review some of the mathematical literature.

Carles [4, 5] proved global wellposedness for a defocusing nonlinearity $F(u)=$ $|u|^{p} u, p<4 /(d-2)$ when the potential $V(x)=|x|^{2} / 2$ is either confining or repulsive, and obtained various wellposedness and blowup results for a focusing nonlinearity $F(u)=-|u|^{p} u$. In [6], he also studied [6] the case of an anisotropic harmonic oscillator with $V(x)=\sum_{j} \delta_{j} x_{j}^{2} / 2, \delta_{j} \in\{1,0,-1\}$.

There has also been interest in more general potentials. The paper 25 proves long-time existence in the presence of a focusing, mass-subcritical nonlinearity $F(u)=-|u|^{p} u, p<4 / d$ when $V(x)$ is merely assumed to grow subquadratically (by which we mean $\partial^{\alpha} V \in L^{\infty}$ for all $|\alpha| \geq 2$ ). More recently, Carles 7 ] considered time-dependent subquadratic potentials $V(t, x)$. Taking initial data in $\Sigma$, he established global existence and uniqueness when $4 / d \leq p<4 /(d-2)$ for the defocusing nonlinearity and $0<p<4 / d$ in the focusing case.

This paper studies the energy-critical problem $p=4 /(d-2)$. While the critical equation still admits a local theory, the duration of local existence obtained by the usual fixed-point argument depends on the profile and not merely on the norm of 
the initial data $u_{0}$. Therefore, one cannot pass directly from local wellposedness to global wellposedness using conservation laws as in the subcritical case. This issue is most evident if we temporarily discard the potential and consider the equation

$$
i \partial_{t} u=-\frac{1}{2} \Delta u+\mu|u|^{\frac{4}{d-2}} u, \quad u(0)=u_{0} \in \dot{H}^{1}\left(\mathbf{R}^{d}\right), \quad d \geq 3,
$$

which has the Hamiltonian

$$
E_{\Delta}(u)=\int \frac{1}{2}|\nabla u|^{2}+\mu \frac{d-2}{d}|u|^{\frac{2 d}{d-2}} d x .
$$

We will refer to this equation in the sequel as the "potential-free", "translationinvariant", or "scale-invariant" problem. Since the spacetime scaling (1.3) preserves both the equation and the $\dot{H}^{1}$ norm of the initial data, the time of existence guaranteed by the local wellposedness theory cannot depend merely on $\left\|u_{0}\right\|_{\dot{H}^{1}}$. One cannot iterate the local existence argument to obtain global existence because with each iteration the solution could conceivably become more concentrated in space while remaining bounded in $\dot{H}^{1}$, so that the duration of local existence could decrease with each iteration. The scale invariance makes the analysis of (1.6) highly nontrivial.

We mention equation (1.6) because the original equation increasingly resembles (1.6) as the initial data concentrates at a point; see sections 4.2 and 5 for more precise statements concerning this sort of limit. Hence, one would expect the essential difficulties in the energy-critical NLS to also manifest themselves in the energycritical harmonic oscillator. Understanding the scale-invariant problem is therefore an important step toward understanding the harmonic oscillator. The last fifteen years have witnessed intensive study of the former, and the following conjecture has been verified in all but a few cases:

Conjecture 1.1. When $\mu=1$, solutions to (1.6) exist globally and scatter. That is, for any $u_{0} \in \dot{H}^{1}\left(\mathbf{R}^{d}\right)$, there exists a unique global solution $u: \mathbf{R} \times \mathbf{R}^{d} \rightarrow \mathbf{C}$ to (1.6) with $u(0)=u_{0}$, and this solution satisfies a spacetime bound

$$
S_{\mathbf{R}}(u):=\int_{\mathbf{R}} \int_{\mathbf{R}^{d}}|u(t, x)|^{\frac{2(d+2)}{d-2}} d x d t \leq C\left(E_{\Delta}\left(u_{0}\right)\right)<\infty .
$$

Moreover, there exist functions $u_{ \pm} \in \dot{H}^{1}\left(\mathbf{R}^{d}\right)$ such that

$$
\lim _{t \rightarrow \pm \infty}\left\|u(t)-e^{ \pm \frac{i t \Delta}{2}} u_{ \pm}\right\|_{\dot{H}^{1}}=0
$$

and the correspondences $u_{0} \mapsto u_{ \pm}\left(u_{0}\right)$ are homeomorphisms of $\dot{H}^{1}$.

When $\mu=-1$, one also has global wellposedness and scattering provided that

$$
E_{\Delta}\left(u_{0}\right)<E_{\Delta}(W), \quad\left\|\nabla u_{0}\right\|_{L^{2}}<\|\nabla W\|_{L^{2}},
$$

where the ground state

$$
W(x)=\frac{1}{\left(1+\frac{2|x|^{2}}{d(d-2)}\right)^{\frac{d-2}{2}}} \in \dot{H}^{1}\left(\mathbf{R}^{d}\right)
$$

solves the elliptic equation $\frac{1}{2} \Delta+|W|^{\frac{4}{d-2}} W=0$.

Theorem 1.1. Conjecture 1.1 holds for the defocusing equation. For the focusing equation, the conjecture holds for radial initial data when $d \geq 3$, and for all initial data when $d \geq 5$.

Proof. See [2, 9, 26, 31] for the defocusing case and [16, 21] for the focusing case. 
One can formulate a similar conjecture for (1.1); however, as the linear propagator is periodic in time, one only expects uniform local-in-time spacetime bounds.

Conjecture 1.2. When $\mu=1$, equation (1.1) is globally wellposed. That is, for each $u_{0} \in \Sigma$ there is a unique global solution $u: \mathbf{R} \times \mathbf{R}^{d} \rightarrow \mathbf{C}$ with $u(0)=u_{0}$. This solution obeys the spacetime bound

$$
S_{I}(u):=\int_{I} \int_{\mathbf{R}^{d}}|u(t, x)|^{\frac{2(d+2)}{d-2}} d x d t \leq C\left(|I|,\left\|u_{0}\right\|_{\Sigma}\right)
$$

for any compact interval $I \subset \mathbf{R}$.

If $\mu=-1$, then the same is true provided also that

$$
E\left(u_{0}\right)<E_{\Delta}(W) \text { and }\left\|\nabla u_{0}\right\|_{L^{2}} \leq\|\nabla W\|_{L^{2}} .
$$

In [23], Killip-Visan-Zhang verifed this conjecture with $\mu=1$ and spherically symmetric initial data. By adapting an argument of Bourgain-Tao for the equation without potential (1.6), they proved that the defocusing problem (1.1) is globally wellposed, and also proved scattering for the repulsive potential. We consider only the confining potential. In this paper, we remove the assumption of spherical symmetry for the defocusing harmonic oscillator, and also establish global wellposedness for the focusing problem under the assumption that Conjecture (1.1) holds for all dimensions. Specifically, we prove

Theorem 1.2. Assume that Conjecture 1.1 holds. Then Conjecture 1.2 holds.

By Theorem 1.1 this result is conditional only in the focusing situation for nonradial data in dimensions 3 and 4 . Moreover, in the focusing case we have essentially the same blowup result as for the potential-free NLS with the same proof as in that case; see 21. We recall the argument in Section 7.

Theorem 1.3 (Blowup). Suppose $\mu=-1$ and $d \geq 3$. If $u_{0} \in \Sigma$ satisfies $E\left(u_{0}\right)<$ $E_{\Delta}(W)$ and $\left\|\nabla u_{0}\right\|_{2} \geq\|\nabla W\|_{2}$, then the solution to (1.1) blows up in finite time.

Mathematically, the energy-critical NLS with quadratic potential has several interesting properties. On one hand, it is a nontrivial variant of the potential-free equation. If the quadratic potential is replaced by a weaker potential, the proof of global wellposedness can sometimes ride on the coat tails of Theorem 1.1. For example, we show in Section 8 that for smooth, bounded potentials with bounded derivative, one obtains global wellposedness by treating the potential as a perturbation to (1.6). Further, the Avron-Herbst formula given in 7 r reduces the problem with a linear potential $V(x)=E x$ to (1.6). On the other hand, the quantum harmonic oscillator enjoys the remarkable property that its linear propagator $e^{-i t H}$ has an explicit integral kernel in the form of Mehler's formula. In view of the preceding remarks, we believe that (1.1) is the most accessible generalization of (1.6) which does not come for free.

Proof outline. The local theory for (1.1) shows that global existence is equivalent to the uniform a priori spacetime bound (1.8). To prove this bound for all solutions, we apply the general strategy of induction on energy pioneered by Bourgain 2 and refined over the years by Colliander-Keel-Staffilani-Takaoka-Tao [9, Keraani [18, Kenig-Merle [16], and others. These arguments proceed roughly as follows. One assumes for a contradiction that Theorem 1.2 fails, and is then faced with two main tasks: 
(1) Prove the existence of a minimal counterexample (where "minimal" will be defined shortly).

(2) Show that this counterexample violates properties obeyed by all solutions.

Let us elaborate a little on these steps. The energy $E(u)$ of a solution (which equals the energy of the initial data) will serve as our induction parameter. By the local wellposedness theory (which we review in Section 31), uniform spacetime bounds hold for all solutions with sufficiently small energy $E(u)$. Assuming that Theorem 1.2 fails, we obtain a positive threshold $0<E_{c}<\infty$ such that (1.8) holds whenever $E(u)<E_{c}$ and fails when $E(u)>E_{c}$. The first step described above would have us construct a solution $u_{c}: I \times \mathbf{R}^{d} \rightarrow \mathbf{C}$ on a bounded interval $I$ with $S_{I}\left(u_{c}\right)=\infty$, and whose energy equals precisely the critical threshold $E_{c}$. Thus "minimal" in our setting refers to minimal energy.

We will carry out a variant of this strategy that is better adapted to what we are trying to prove. Since the spacetime estimates of interest are local-in-time, it suffices to prevent the blowup of spacetime norm on arbitrarily small time intervals. That is, we need only show that for each energy $E>0$, there exists some $L=L(E)>0$ so that $S_{I}(u) \leq C(E)$ whenever $E(u) \leq E$ and $|I| \leq L$. To prove this statement, we work not with minimal-energy blowup solutions directly but rather with the PalaisSmale compactness theorem (Proposition 6.1) that would beget such solutions. Our argument will ultimately reduce the question of global wellposedness for (1.1) to that of global wellposedness and scattering for the potential-free equation (1.6). In effect, we shall discover that the only scenario where blowup could possibly occur is when the solution is highly concentrated at a point and behaves like a solution to (1.6).

This paradigm of recovering the potential-free NLS in certain limiting regimes is by now well-known and has been applied to the study of other equations. See [19, 20, 14, 13, 12, 24] for adaptations to gKdV, Klein-Gordon, and NLS in various domains and manifolds. While the particulars are unique to each case, a common key step is to prove an appropriate compactness theorem in the style of Proposition 6.1. As in the previous work, our proof of that proposition uses three main ingredients.

The first prerequisite is a local wellposedness theory that gives local existence and uniqueness as well as stability of solutions with respect to perturbations of the initial data or the equation itself. In our case, local wellposedness will follow from familiar arguments employing the dispersive estimate satisfied by the linear propagator $e^{-i t H}$, as well the fractional product and chain rules for the operators $H^{\gamma}, \gamma \geq 0$. We review the relevant results in Section 3 .

We also need a linear profile decomposition for the Strichartz inequality

$$
\left\|e^{-i t H} f\right\|_{L_{t, x}^{\frac{2(d+2)}{d-2}}} \lesssim\left\|H^{\frac{1}{2}} f\right\|_{L_{x}^{2}} .
$$

Such a decomposition in the context of energy-critical Schrödinger equations was first proved by Keraani [17] in the translation-invariant setting for the free particle Hamiltonian $H=-\Delta$, and quantifies the manner in which a sequence of functions $f_{n}$ with $\left\|H^{1 / 2} f_{n}\right\|_{L^{2}}$ bounded may fail to produce a subsequence of $e^{-i t H} f_{n}$ converging in the spacetime norm. The defect of compactness arises in Keraani's case from a noncompact group of symmetries of the inequality (1.9), which includes spatial translations and scaling. In our setting, there are no obvious symmetries of (1.9); nonetheless, compactness can fail and in Section 4 we formulate a profile decomposition for (1.9) when $H$ is the Hamiltonian of the harmonic oscillator. 
The final ingredient is an analysis of (1.1) when the initial data is highly concentrated in space, corresponding to a single profile in the linear profile decomposition just discussed. In Section 5 we show that blowup cannot occur in this regime. The basic idea is that while the solution to (1.1) remains highly localized in space, it can be well-approximated up to a phase factor by the corresponding solution to the scale-invariant energy-critical NLS

$$
\left(i \partial_{t}+\frac{1}{2} \Delta\right) u= \pm|u|^{\frac{4}{d-2}} u .
$$

By the time this approximation breaks down, the solution to the original equation will have dispersed and can instead be approximated by a solution to the linear equation $\left(i \partial_{t}-H\right) u=0$. We use as a black box the nontrivial fact (which is still a conjecture in several cases) that solutions to (1.6) obey global spacetime bounds. By stability theory, the spacetime bounds for the approximations will be transferred to the solution for the original equation and will therefore preclude blowup.

We have chosen to focus on the concrete potential $V(x)=\frac{1}{2}|x|^{2}$ mainly for concreteness. In a forthcoming paper we will indicate how to extend the main result to a more general class of subquadratic potentials.

Acknowledgements. The author is indebted to his advisors Rowan Killip and Monica Visan for their helpful discussions as well as their feedback on the paper. This work was supported in part by NSF grants DMS-0838680 (RTG), DMS1265868 (PI R. Killip), DMS-0901166, and DMS-1161396 (both PI M. Visan).

\section{Preliminaries}

2.1. Notation and basic estimates. We write $X \lesssim Y$ to mean $X \leq C Y$ for some constant $C$. Similarly $X \sim Y$ means $X \lesssim Y$ and $Y \lesssim X$. Denote by $L^{p}\left(\mathbf{R}^{d}\right)$ the Banach space of functions $f: \mathbf{R}^{d} \rightarrow \mathbf{C}$ with finite norm

$$
\|f\|_{L^{p}\left(\mathbf{R}^{d}\right)}=\left(\int_{\mathbf{R}^{d}}|f|^{p} d x\right)^{\frac{1}{p}}
$$

We will sometimes use the more compact notation $\|f\|_{p}$. If $I \subset \mathbf{R}^{d}$ is an interval, the mixed Lebesgue norms on $I \times \mathbf{R}^{d}$ are defined by

$$
\|f\|_{L_{t}^{q} L_{x}^{r}\left(I \times \mathbf{R}^{d}\right)}=\left(\int_{I}\left(\int_{\mathbf{R}^{d}}|f(t, x)|^{r} d x\right)^{\frac{q}{r}} d t\right)^{\frac{1}{q}}=\|f(t)\|_{L_{t}^{q}\left(I ; L_{x}^{r}\left(\mathbf{R}^{d}\right)\right)},
$$

where one regards $f(t)=f(t, \cdot)$ as a function from $I$ to $L^{r}\left(\mathbf{R}^{d}\right)$.

The operator $H=-\frac{1}{2} \Delta+\frac{1}{2}|x|^{2}$ is positive on $L^{2}\left(\mathbf{R}^{d}\right)$. Its associated heat kernel is given by Mehler's formula [10]:

$$
e^{-t H}(x, y)=e^{\tilde{\gamma}(t)\left(x^{2}+y^{2}\right)} e^{\frac{\sinh (t) \Delta}{2}}(x, y)
$$

where

$$
\tilde{\gamma}(t)=\frac{1-\cosh t}{2 \sinh t}=-\frac{t}{4}+O\left(t^{3}\right) \quad \text { as } \quad t \rightarrow 0 .
$$

By analytic continuation, the associated one-parameter unitary group has the integral kernel

$$
e^{-i t H} f(x)=\frac{1}{(2 \pi i \sin t)^{\frac{d}{2}}} \int e^{\frac{i}{\sin t}\left(\frac{x^{2}+y^{2}}{2} \cos t-x y\right)} f(y) d y .
$$


Comparing this to the well-known free propagator

$$
e^{\frac{i t \Delta}{2}} f(x)=\frac{1}{(2 \pi i t)^{\frac{d}{2}}} \int e^{\frac{i|x-y|^{2}}{2 t}} f(y) d y,
$$

we obtain the relation

$$
e^{-i t H} f=e^{i \gamma(t)|x|^{2}} e^{\frac{i \sin (t) \Delta}{2}}\left(e^{i \gamma(t)|x|^{2}} f\right)
$$

where

$$
\gamma(t)=\frac{\cos t-1}{2 \sin t}=-\frac{t}{4}+O\left(t^{3}\right) \quad \text { as } \quad t \rightarrow 0 .
$$

Mehler's formula immediately implies the local-in-time dispersive estimate

$$
\left\|e^{-i t H} f\right\|_{L_{x}^{\infty}} \lesssim|\sin t|^{-\frac{d}{2}}\|f\|_{L^{1}}
$$

For $d \geq 3$, call a pair of exponents $(q, r)$ admissible if $q \geq 2$ and $\frac{2}{q}+\frac{d}{r}=\frac{d}{2}$. Write

$$
\|f\|_{S(I)}=\|f\|_{L_{t}^{\infty} L_{x}^{2}}+\|f\|_{L_{t}^{2} L_{x}^{\frac{2 d}{d-2}}}
$$

with all norms taken over the spacetime slab $I \times \mathbf{R}^{d}$. By interpolation, we see that this norm controls the $L_{t}^{q} L_{x}^{r}$ norm for all other admissible pairs. We let

$$
\|F\|_{N(I)}=\inf \left\{\left\|F_{1}\right\|_{L_{t}^{q_{1}^{\prime}} L_{x}^{r_{1}^{\prime}}}+\left\|F_{2}\right\|_{L_{t}^{q_{2}^{\prime}} L_{x}^{r_{2}^{\prime}}}:\left(q_{k}, r_{k}\right) \text { admissible, } F=F_{1}+F_{2}\right\},
$$

where $\left(q_{k}^{\prime}, r_{k}^{\prime}\right)$ is the Hölder dual to $\left(q_{k}, r_{k}\right)$.

Lemma 2.1 (Strichartz estimates). Let I be a compact time interval containing $t_{0}$, and let $u: I \times \mathbf{R}^{d} \rightarrow \mathbf{C}$ be a solution to the inhomogeneous Schrödinger equation

$$
\left(i \partial_{t}-H\right) u=F .
$$

Then there is a constant $C=C(|I|)$, depending only on the length of the interval, such that

$$
\|u\|_{S(I)} \leq C\left(\left\|u\left(t_{0}\right)\right\|_{L^{2}}+\|F\|_{N(I)}\right) .
$$

Proof. This follows from the dispersive estimate (2.5), the unitarity of $e^{-i t H}$ on $L^{2}$, and general considerations; see [15]. By partitioning time into unit intervals, we see that the constant $C$ grows at worst like $|I|^{\frac{1}{2}}$ (which corresponds to the time exponent $q=2$ ).

It will be convenient to introduce the operators which represent the time evolution of the momentum and position operators under the linear propagator. These are well-known in the literature and were used in [4] or 23, for example. We define

$$
\begin{aligned}
& P(t)=e^{i t H} i \nabla e^{-i t H}=i \nabla \cos t-x \sin t \\
& X(t)=e^{i t H} x e^{-i t H}=i \nabla \sin t+x \cos t .
\end{aligned}
$$

One easily verifies the identity

$$
\|P(t) f\|_{L^{2}}^{2}+\|X(t) f\|_{L^{2}}^{2}=\|P(t) f\|_{L^{2}}^{2}+\left\|P\left(t+\frac{\pi}{2}\right) f\right\|_{L^{2}}^{2}=\|f\|_{\Sigma}^{2} .
$$

We use the fractional powers $H^{\gamma}$ of the operator $H$, defined via the Borel functional calculus, as a substitute for the usual derivative $(-\Delta)^{\gamma}$, which does not commute with the linear propagator $e^{-i t H}$. We have trivially that

$$
\left\|H^{\frac{1}{2}} f\right\|_{L^{2}} \sim\left\|(-\Delta)^{\frac{1}{2}} f\right\|_{L^{2}}+\||x| f\|_{L^{2}} \sim\|f\|_{\Sigma} .
$$


Perhaps less obvious is the fact that this equivalence generalizes to other $L^{p}$ norms and other powers of $H$. Using complex interpolation, Killip, Visan, and Zhang showed that this is the case:

Lemma 2.2 ([23, Lemma 2.7]). For $0 \leq \gamma \leq 1$ and $1<p<\infty$, one has

$$
\left\|H^{\gamma} f\right\|_{L^{p}\left(\mathbf{R}^{d}\right)} \sim\left\|(-\Delta)^{\gamma} f\right\|_{L^{p}\left(\mathbf{R}^{d}\right)}+\left\||x|^{2 \gamma} f\right\|_{L^{p}\left(\mathbf{R}^{d}\right)} .
$$

As a consequence, $H^{\gamma}$ inherits many properties of $(-\Delta)^{\gamma}$, including Sobolev embedding:

Lemma 2.3 ([23, Lemma 2.8]). Suppose $\gamma \in[0,1]$ and $1<p<\frac{d}{2 \gamma}$, and define $p^{*}$ by $\frac{1}{p^{*}}=\frac{1}{p}-\frac{2 \gamma}{d}$. Then

$$
\|f\|_{L^{p^{*}\left(\mathbf{R}^{d}\right)}} \lesssim\left\|H^{\gamma} f\right\|_{L^{p}\left(\mathbf{R}^{d}\right)} .
$$

Similarly, the fractional chain and product rules carry over to the current setting:

Corollary 2.4 ([23, Proposition 2.10]). Let $F(z)=|z|^{\frac{4}{d-2}} z$. For any $0 \leq \gamma \leq \frac{1}{2}$ and $1<p<\infty$,

$$
\left\|H^{\gamma} F(u)\right\|_{L^{p}\left(\mathbf{R}^{d}\right)} \lesssim\left\|F^{\prime}(u)\right\|_{L^{p_{0}}\left(\mathbf{R}^{d}\right)}\left\|H^{\gamma} f\right\|_{L^{p_{1}}\left(\mathbf{R}^{d}\right)}
$$

for all $p_{0}, p_{1} \in(1, \infty)$ with $p^{-1}=p_{0}^{-1}+p_{1}^{-1}$.

Using Lemma 2.2 and the Christ-Weinstein fractional product rule for $(-\Delta)^{\gamma}$ (e.g. [30]), we obtain

Corollary 2.5. For $\gamma \in(0,1], r, p_{i}, q_{i} \in(1, \infty)$ with $r^{-1}=p_{i}^{-1}+q_{i}^{-1}, i=1,2$, we have

$$
\left\|H^{\gamma}(f g)\right\|_{r} \lesssim\left\|H^{\gamma} f\right\|_{p_{1}}\|g\|_{q_{1}}+\|f\|_{p_{2}}\left\|H^{\gamma} g\right\|_{q_{2}} .
$$

The exponent $\gamma=\frac{1}{2}$ is particularly relevant to us, and it will be convenient to use the notation $L_{t}^{q} \Sigma_{x}^{r}\left(I \times \mathbf{R}^{d}\right)$ for the space of functions $f$ with norm

$$
\|f\|_{L_{t}^{q} \Sigma_{x}^{r}}=\left\|H^{\frac{1}{2}} f\right\|_{L_{t}^{q} L_{x}^{r}}
$$

The superscript on $\Sigma$ is assumed to be 2 if omitted. We shall need the following refinement of Fatou's Lemma due to Brézis and Lieb:

Lemma 2.6 (Refined Fatou [3]). Fix $1 \leq p<\infty$, and suppose $f_{n}$ is a sequence of functions in $L^{p}\left(\mathbf{R}^{d}\right)$ such that $\sup _{n}\left\|f_{n}\right\|_{p}<\infty$ and $f_{n} \rightarrow f$ pointwise. Then

$$
\left.\lim _{n \rightarrow \infty} \int_{\mathbf{R}^{d}}|| f_{n}\right|^{p}-\left|f_{n}-f\right|^{p}-|f|^{p} \mid d x=0 .
$$

Finally, we record an analogue of the Hörmander-Mikhlin Fourier multiplier theorem proved by Hebisch [11. It enables a Littlewood-Paley theory adapted to $H$, as discussed in the next section.

Theorem 2.1. If $F: \mathbf{R} \rightarrow \mathbf{C}$ is a bounded function which obeys the derivative estimates

$$
\left|\partial^{k} F(\lambda)\right| \lesssim_{k}|\lambda|^{-k} \quad \text { for all } \quad 0 \leq k \leq \frac{d}{2}+1,
$$

then the operator $F(H)$, defined initially on $L^{2}$ via the Borel functional calculus, is bounded from $L^{p}$ to $L^{p}$ for all $1<p<\infty$. 
2.2. Littlewood-Paley theory. Owing largely to Theorem 2.1, we can import the basic results of Littlewood-Paley theory with little effort, the only change being that one replaces Fourier multipliers with spectral multipliers. We fashion two kinds of Littlewood-Paley projections, one using compactly supported bump functions, and the other based on the heat kernel of $H$. The parabolic maximum principle implies that

$$
0 \leq e^{-t H}(x, y) \leq e^{\frac{t \Delta}{2}}(x, y)=\frac{1}{(2 \pi t)^{d / 2}} e^{-\frac{|x-y|^{2}}{2 t}} .
$$

Fix a smooth function $\varphi$ supported in $|\lambda| \leq 2$ with $\varphi(\lambda)=1$ for $|\lambda| \leq 1$, and let $\psi(\lambda)=\varphi(\lambda)-\varphi(2 \lambda)$. For each dyadic number $N \in 2^{\mathbf{Z}}$, which we will often refer to as "frequency," define

$$
\begin{array}{ll}
P_{\leq N}^{H}=\varphi\left(\sqrt{H / N^{2}}\right), & P_{N}^{H}=\psi\left(\sqrt{H / N^{2}}\right), \\
\tilde{P}_{\leq N}^{H}=e^{-H / N^{2}}, & \tilde{P}_{N}^{H}=e^{-H / N^{2}}-e^{-4 H / N^{2}} .
\end{array}
$$

The associated operators $P_{<N}^{H}, P_{>N}^{H}$, etc. are defined in the usual manner.

Remark. As the spectrum of $H$ is bounded away from 0 , by choosing $\varphi$ appropriately we can arrange for $P_{<1}=0$; thus we will only consider frequencies $N \geq 1$.

Similarly, let

$$
\begin{array}{ll}
P_{\leq N}^{\Delta}=\varphi\left(\sqrt{-\Delta / N^{2}}\right) & P_{N}^{\Delta}=\psi\left(\sqrt{-\Delta / N^{2}}\right), \\
\tilde{P}_{\leq N}^{\Delta}=e^{\Delta / 2 N^{2}} & \tilde{P}_{N}^{\Delta}=e^{\Delta / 2 N^{2}}-e^{2 \Delta / N^{2}} .
\end{array}
$$

denote the classical Littlewood-Paley projections. From the maximum principle we obtain the pointwise bound

$$
\left|\tilde{P}_{N}^{H} f(x)\right|+\left|\tilde{P}_{\leq N}^{H} f(x)\right| \lesssim \tilde{P}_{\leq N}^{\Delta}|f|(x)+\tilde{P}_{\leq N / 2}^{\Delta}|f|(x) .
$$

To reduce clutter we usually suppress the superscripts $H$ and $\Delta$ when it is clear from the context which type of projection we are using. For the rest of this section, $P_{\leq N}$ and $P_{N}$ denote $P_{\leq N}^{H}$ and $P_{N}^{H}$, respectively.

Lemma 2.7 (Bernstein estimates). For $f \in C_{c}^{\infty}\left(\mathbf{R}^{d}\right), 1<p \leq q<\infty, s \geq 0$, one has the Bernstein inequalities

$$
\begin{gathered}
\left\|P_{\leq N} f\right\|_{p} \lesssim\left\|\tilde{P}_{\leq N} f\right\|_{p}, \quad\left\|P_{N} f\right\|_{p} \lesssim\left\|\tilde{P}_{N} f\right\|_{p} \\
\left\|P_{\leq N} f\right\|_{p}+\left\|P_{N} f\right\|_{p}+\left\|\tilde{P}_{\leq N} f\right\|_{p}+\left\|\tilde{P}_{N} f\right\|_{p} \lesssim\|f\|_{p} \\
\left\|P_{\leq N} f\right\|_{q}+\left\|P_{N} f\right\|_{q}+\left\|\tilde{P}_{\leq N} f\right\|_{q}+\left\|\tilde{P}_{N} f\right\|_{q} \lesssim N^{\frac{d}{p}-\frac{d}{q}}\|f\|_{p} \\
N^{2 s}\left\|P_{N} f\right\|_{p} \sim\left\|H^{s} P_{N} f\right\|_{p} \\
\left\|P_{>N} f\right\|_{p} \lesssim N^{-2 s}\left\|H^{s} P_{>N} f\right\|_{p} .
\end{gathered}
$$

In (2.13), the estimates for $\tilde{P}_{\leq N} f$ and $\tilde{P}_{N} f$ also hold when $p=1, q=\infty$. Further,

$$
f=\sum_{N} P_{N} f=\sum_{N} \tilde{P}_{N} f
$$

where the series converge in $L^{p}, 1<p<\infty$. Finally, we have the square function estimate

$$
\|f\|_{p} \sim\left\|\left(\sum_{N}\left|P_{N} f\right|^{2}\right)^{1 / 2}\right\|_{p}
$$


Proof. The estimates (2.11) follow immediately from Theorem 2.1, To see (2.12), observe that the functions $\varphi\left(\sqrt{\cdot / N^{2}}\right), e^{-\cdot / N^{2}}$ satisfy the hypotheses of Theorem 2.1 uniformly in $N$. Next use (2.7) together with Young's convolution inequality to get

$$
\left\|\tilde{P}_{\leq N} f\right\|_{q}+\left\|\tilde{P}_{N} f\right\|_{q} \lesssim N^{\frac{d}{q}-\frac{d}{p}}\|f\|_{p} \quad \text { for } 1 \leq p \leq q \leq \infty .
$$

From (2.11) we obtain the rest of (2.13). Now consider (2.14). Let $\tilde{\psi}$ be a fattened version of $\psi$ so that $\tilde{\psi}=1$ on the support of $\psi$. Put $F(\lambda)=\lambda^{s} \tilde{\psi}(\sqrt{\lambda})$. By Theorem 2.1, the relation $\psi=\tilde{\psi} \psi$, and the functional calculus,

$$
\left\|N^{-2 s} H^{s} P_{N} f\right\|_{p}=\left\|F\left(H / N^{2}\right) P_{N} f\right\|_{p} \lesssim\left\|P_{N} f\right\|_{p} .
$$

The reverse inequality follows by considering $F(x)=\lambda^{-s} \tilde{\psi}(\lambda)$.

We turn to (2.16). The equality holds in $L^{2}$ by the functional calculus and the fact that the spectrum of $H$ is bounded away from 0 . For $p \neq 2$, choose $q$ and $0<\theta<1$ so that $p^{-1}=2^{-1}(1-\theta)+q^{-1} \theta$. By (2.12), the partial sum operators

$$
S_{N_{0}, N_{1}}=\sum_{N_{0}<N \leq N_{1}} P_{N}, \quad \tilde{S}_{N_{0}, N_{1}}=\sum_{N_{0}<N \leq N_{1}} \tilde{P}_{N}
$$

are bounded on every $L^{p}, 1<p<\infty$, uniformly in $N_{0}, N_{1}$. Thus by Hölder's inequality,

$$
\left\|f-S_{N_{0}, N_{1}} f\right\|_{p} \leq\left\|f-S_{N_{0}, N_{1}} f\right\|_{2}^{1-\theta}\left\|f-S_{N_{0}, N_{1}} f\right\|_{q}^{\theta} \rightarrow 0 \text { as } N_{0} \rightarrow 0, N_{1} \rightarrow \infty,
$$

and similarly for the partial sums $\tilde{S}_{N_{0}, N_{1}} f$. The estimate (2.15) follows from (2.12), (2.14), and the decomposition $P_{>N} f=\sum_{M>N} P_{M} f$.

To prove the square function estimate, run the usual Khintchine's inequality argument using Theorem 2.1 in place of the Mikhlin multiplier theorem.

2.3. Local smoothing. The following local smoothing lemma and its corollary will be needed when proving properties of the nonlinear profile decomposition in Section 6

Lemma 2.8. If $u=e^{-i t H} \phi, \phi \in \Sigma\left(\mathbf{R}^{d}\right)$, then

$$
\int_{I} \int_{\mathbf{R}^{d}}|\nabla u(x)|^{2}\left\langle R^{-1}(x-z)\right\rangle^{-3} d x d t \lesssim R(1+|I|)\|u\|_{L_{t}^{\infty} L_{x}^{2}}\left\|H^{1 / 2} u\right\|_{L_{t}^{\infty} L_{x}^{2}}
$$

with the constant independent of $z \in \mathbf{R}^{d}$ and $R>0$.

Proof. We recall the Morawetz identity. Let $a$ be a sufficiently smooth function of $x$; then for any $u$ satisfying the linear equation $i \partial_{t} u=\left(-\frac{1}{2} \Delta+V\right) u$, one has

$$
\begin{aligned}
\partial_{t} \int \nabla a \cdot \operatorname{Im}(\bar{u} \nabla u) d x= & \int a_{j k} \operatorname{Re}\left(u_{j} \bar{u}_{k}\right) d x-\frac{1}{4} \int|u|^{2} a_{j j k k} d x \\
& -\frac{1}{2} \int|u|^{2} \nabla a \cdot \nabla V d x
\end{aligned}
$$

We use this identity with $a(x)=\left\langle R^{-1}(x-z)\right\rangle$ and $V=\frac{1}{2}|x|^{2}$, and compute

$$
\begin{aligned}
a_{j}(x) & =\frac{R^{-2}\left(x_{j}-z_{j}\right)}{\left\langle R^{-1}(x-z)\right\rangle}, a_{j k}(x)=R^{-2}\left[\frac{\delta_{j k}}{\left\langle R^{-1}(x-z)\right\rangle}-\frac{R^{-2}\left(x_{j}-z_{j}\right)\left(x_{k}-z_{k}\right)}{\left\langle R^{-1}(x-z)\right\rangle^{3}}\right] \\
\Delta^{2} a(x) & \leq-\frac{15 R^{-4}}{\left\langle R^{-1}(x-z)\right\rangle^{7}} .
\end{aligned}
$$


As $\Delta^{2} a \leq 0$, the right side of (2.19) is bounded below by

$$
\begin{aligned}
& R^{-2} \int\left\langle R^{-1}(x-z)\right\rangle^{-1}\left[|\nabla u|^{2}-\left|\frac{R^{-1}(x-z)}{\left\langle R^{-1}(x-z)\right\rangle} \cdot \nabla u\right|^{2}\right] d x-\frac{1}{2 R} \int|u|^{2} \frac{R^{-1}(x-z)}{\left\langle R^{-1}(x-z)\right\rangle} \cdot x d x \\
& \geq R^{-2} \int|\nabla u(x)|^{2}\left\langle R^{-1}(x-z)\right\rangle^{-3} d x-\frac{R^{-1}}{2} \int|u|^{2}|x| d x .
\end{aligned}
$$

Integrating in time and applying Cauchy-Schwarz, we get

$$
\begin{aligned}
R^{-2} \int_{I} \int_{\mathbf{R}^{d}} & \left\langle R^{-1}(x-z)\right\rangle^{-3}|\nabla u(t, x)|^{2} d x d t \\
& \lesssim \sup _{t \in I} R^{-1} \int \frac{R^{-1}(x-z)}{\left\langle R^{-1}(x-z)\right\rangle}|u(t, x)||\nabla u(t, x)| d x+\frac{1}{2 R} \int_{I} \int_{\mathbf{R}^{d}}|x||u|^{2} d x d t \\
& \lesssim R^{-1}(1+|I|)\|u\|_{L_{t}^{\infty} L_{x}^{2}}\left\|H^{1 / 2} u\right\|_{L_{t}^{\infty} L_{x}^{2} .}
\end{aligned}
$$

This completes the proof of the lemma.

Corollary 2.9. Fix $\phi \in \Sigma\left(\mathbf{R}^{d}\right)$. Then for all $T, R \leq 1$, we have

$$
\left\|\nabla e^{-i t H} \phi\right\|_{L_{t, x}^{2}\left(\left|t-t_{0}\right| \leq T,\left|x-x_{0}\right| \leq R\right)} \lesssim T^{\frac{2}{3(d+2)}} R^{\frac{3 d+2}{3(d+2)}}\|\phi\|_{\Sigma}^{\frac{2}{3}}\left\|e^{-i t H} \phi\right\|_{L_{t, x}^{\frac{2(d+2)}{3}}}^{\frac{1}{2-2}} .
$$

When $d=3$, we also have

$$
\left\|\nabla e^{-i t H} \phi\right\|_{L_{t}^{\frac{10}{3}} L_{x}^{\frac{15}{7}}\left(\left|t-t_{0}\right| \leq T,\left|x-x_{0}\right| \leq R\right)} \lesssim T^{\frac{23}{180}} R^{\frac{11}{45}}\left\|e^{-i t H} \phi\right\|_{L_{t, x}^{10}}^{\frac{5}{48}}\|\phi\|_{\Sigma}^{\frac{43}{48}}
$$

Proof. The proofs are fairly standard (see 32] or [24]), and we present just the proof of the second claim, which is slightly more involved. Let $E$ the region $\left\{\left|t-t_{0}\right| \leq\right.$ $\left.T,\left|x-x_{0}\right| \leq R\right\}$. Norms which do not specify the region of integration are taken over the spacetime slab $\left\{\left|t-t_{0}\right| \leq T\right\} \times \mathbf{R}^{3}$. By Hölder,

$$
\left\|\nabla e^{-i t H} \phi\right\|_{L_{t}^{\frac{10}{3}} L_{x}^{\frac{15}{4}}(E)} \leq\left\|\nabla e^{-i t H} \phi\right\|_{L_{t, x}^{2}(E)}^{\frac{1}{3}}\left\|\nabla e^{-i t H} \phi\right\|_{L_{t}^{5} L_{x}^{\frac{20}{9}}(E)}^{\frac{2}{3}} .
$$

By Hölder and Strichartz,

$$
\left\|\nabla e^{-i t H} \phi\right\|_{L_{t}^{5} L_{x}^{\frac{20}{9}}(E)} \lesssim T^{\frac{1}{8}}\left\|\nabla e^{-i t H} \phi\right\|_{L_{t}^{\frac{40}{3}} L_{x}^{\frac{20}{9}}} \lesssim T^{\frac{1}{8}}\|\phi\|_{\Sigma}
$$

We now estimate $\left\|\nabla e^{-i t H} \phi\right\|_{L_{t, x}^{2}}$. Let $N \in 2^{\mathbf{N}}$ be a dyadic number to be chosen later, and decompose

$$
\left\|\nabla e^{-i t H} \phi\right\|_{L_{t, x}^{2}(E)} \leq\left\|\nabla e^{-i t H} P_{\leq N}^{H} \phi\right\|_{L_{t, x}^{2}(E)}+\left\|\nabla e^{-i t H} P_{>N}^{H} \phi\right\|_{L_{t, x}^{2}(E)} .
$$

For the low frequency piece, apply Hölder and the Bernstein inequalities to obtain

$$
\left\|\nabla e^{-i t H} P_{\leq N}^{H} \phi\right\|_{L_{t, x}^{2}} \lesssim T^{\frac{2}{5}} R^{\frac{6}{5}}\left\|\nabla e^{-i t H} P_{\leq N}^{H} \phi\right\|_{L_{t, x}^{10}} \lesssim T^{\frac{2}{5}} R^{\frac{6}{5}} N\left\|e^{-i t H} \phi\right\|_{L_{t, x}^{10}} .
$$

For the high-frequency piece, apply local smoothing and Bernstein:

$$
\left\|\nabla e^{-i t H} P_{>N}^{H} \phi\right\|_{L_{t, x}^{2}} \lesssim R^{\frac{1}{2}}\left\|P_{>N}^{H} \phi\right\|_{L^{2}}^{\frac{1}{2}}\left\|H^{\frac{1}{2}} \phi\right\|_{\Sigma}^{\frac{1}{2}} \lesssim R^{\frac{1}{2}} N^{-\frac{1}{2}}\|\phi\|_{\Sigma}
$$

Optimizing in $N$, we obtain

$$
\left\|\nabla e^{-i t H} \phi\right\|_{L_{t, x}^{2}} \lesssim T^{\frac{2}{15}} R^{\frac{11}{15}}\left\|e^{-i t H} \phi\right\|_{L_{t, x}^{10}}^{\frac{1}{3}}\|\phi\|_{\Sigma}^{\frac{2}{3}} .
$$

Combining this estimate with (2.20) yields the conclusion of the corollary. 


\section{LOCAL THEORY}

We record some standard results concerning local-wellposedness for (1.1). These are direct analogues of the theory for the scale-invariant equation (1.6). By Lemma2.3 and Corollaries 2.4 and 2.5, we can use essentially the same proofs as in that case. We refer the reader to 22 for those proofs.

Proposition 3.1 (Local wellposedness). Let $u_{0} \in \Sigma\left(\mathbf{R}^{d}\right)$ and fix a compact time interval $0 \in I \subset \mathbf{R}$. Then there exists a constant $\eta_{0}=\eta_{0}(d,|I|)$ such that whenever $\eta<\eta_{0}$ and

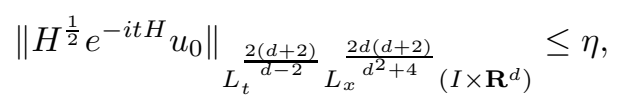

there exists a unique solution $u: I \times \mathbf{R}^{d} \rightarrow \mathbf{C}$ to (1.1) which satisfies the bounds

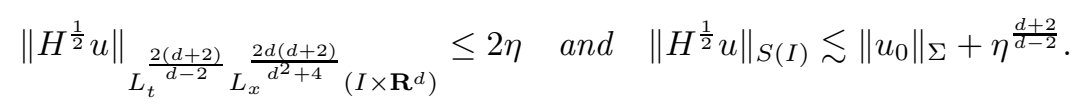

Corollary 3.2 (Blowup criterion). Suppose $u:\left(T_{\min }, T_{\max }\right) \times \mathbf{R}^{d} \rightarrow \mathbf{C}$ is a maximal lifespan solution to (1.1), and fix $T_{\min }<t_{0}<T_{\max }$. If $T_{\max }<\infty$, then

$$
\|u\|_{L_{t, x}^{\frac{2(d+2)}{d-2}}\left(\left[t_{0}, T_{\max }\right)\right)}=\infty .
$$

If $T_{\min }>-\infty$, then

$$
\|u\|_{L_{t, x}^{\frac{2(d+2)}{d-2}}\left(\left(T_{\min }, t_{0}\right]\right)}=\infty .
$$

Proposition 3.3 (Stability). Fix $t_{0} \in I \subset \mathbf{R}$ an interval of unit length and let $\tilde{u}: I \times \mathbf{R}^{d} \rightarrow \mathbf{C}$ be an approximate solution to (1.1) in the sense that

$$
i \partial_{t} \tilde{u}=H u \pm|\tilde{u}|^{\frac{4}{d-2}} \tilde{u}+e
$$

for some function e. Assume that

$$
\|\tilde{u}\|_{L_{t, x} \frac{2(d+2)}{d-2}} \leq L, \quad\left\|H^{\frac{1}{2}} u\right\|_{L_{t}^{\infty} L_{x}^{2}} \leq E,
$$

and that for some $0<\varepsilon<\varepsilon_{0}(E, L)$ one has

$$
\left\|\tilde{u}\left(t_{0}\right)-u_{0}\right\|_{\Sigma}+\left\|H^{\frac{1}{2}} e\right\|_{N(I)} \leq \varepsilon
$$

Then there exists a unique solution $u: I \times \mathbf{R}^{d} \rightarrow \mathbf{C}$ to (1.1) with $u\left(t_{0}\right)=u_{0}$ and which further satisfies the estimates

$$
\|\tilde{u}-u\|_{L_{t, x}^{\frac{2(d+2)}{d-2}}}+\left\|H^{\frac{1}{2}}(\tilde{u}-u)\right\|_{S(I)} \lesssim C(E, L) \varepsilon^{c}
$$

where $0<c=c(d)<1$ and $C(E, L)$ is a function which is nondecreasing in each variable.

\section{Concentration compactness}

In this section we discuss some concentration compactness results for the Strichartz inequality

$$
\left\|e^{-i t H} f\right\|_{L_{t, x}^{\frac{2(d+2)}{d-2}}\left(I \times \mathbf{R}^{d}\right)} \leq C(|I|, d)\|f\|_{\Sigma},
$$

culminating in the linear profile decomposition of Proposition 4.14. Our profile decomposition resembles that of Keraani [17 in the sense that each profile lives at a well-defined location in spacetime and has a characteristic length scale. But 
since the function space $\Sigma$ lacks both translation and scaling symmetry, the precise definitions of our profiles will be more complicated.

Keraani considered the analogous Strichartz estimate

$$
\left\|e^{i t \Delta} f\right\|_{L_{t, x}^{\frac{2(d+2)}{d-2}}\left(\mathbf{R} \times \mathbf{R}^{d}\right)} \lesssim\|f\|_{\dot{H}^{1}\left(\mathbf{R}^{d}\right)} .
$$

Recall that in that situation, if $f_{n}$ is a bounded sequence in $\dot{H}^{1}$ with nontrivial linear evolution, then one has a decomposition $f_{n}=\phi_{n}+r_{n}$ where $\phi_{n}=e^{i t_{n} \Delta} G_{n} \phi, G_{n}$ are certain unitary scaling and translation operators on $\dot{H}^{1}$ (defined as in (4.1)), and $\phi$ is a weak limit of $G_{n}^{-1} e^{-i t_{n} \Delta} f_{n}$ in $\dot{H}^{1}$. The "bubble" $\phi_{n}$ is nontrivial and decouples from the remainder $r_{n}$ in various norms. By applying this decomposition inductively to the remainder $r_{n}$, one obtains the full collection of profiles constituting $f_{n}$.

We follow the general presentation in [22, 32]. Let $f_{n} \in \Sigma$ be a bounded sequence. Using a variant of Keraani's argument, we seek to obtain an $\dot{H}^{1}$-weak limit $\phi$ in terms of $f_{n}$ and write $f_{n}=\phi_{n}+r_{n}$ where $\phi_{n}$ is defined analogously as before by "moving the operators onto $f_{n}$." However, we need to modify this procedure in light of two issues.

The first problem is that while $f_{n}$ belong to $\Sigma$, an $\dot{H}^{1}$ weak limit of a sequence like $G_{n}^{-1} e^{i t_{n} H} f_{n}$ need only belong to $\dot{H}^{1}$. Indeed, the $\dot{H}^{1}$ isometries $G_{n}^{-1}$ will in general have unbounded norm as operators on $\Sigma$ because of the $|x|^{2}$ spatial weight, which penalizes very wide functions. To define $\phi_{n}$, we need to introduce suitably chosen spatial cutoffs to obtain functions in $\Sigma$.

Secondly, to establish the various orthogonality assertions we must understand how the linear propagator $e^{-i t H}$ interacts with the $\dot{H}^{1}$ symmetries of translation and scaling in certain limits. We study this interaction in Section 4.2. In particular, the convergence lemmas proved there serve as a substitute for the scaling relation

$$
e^{i t \Delta} G_{n}=G_{n} e^{i N_{n}^{2} t \Delta} \quad \text { where } \quad G_{n} \phi=N_{n}^{\frac{d-2}{2}} \phi\left(N_{n}\left(\cdot-x_{n}\right)\right) .
$$

They can also be regarded as a precise form of the heuristic stated in the introduction that as we scale the initial data to concentrate at a point $x_{0}$, the potential $V(x)=|x|^{2} / 2$ can be treated over short time intervals as essentially equal to the constant potential $V\left(x_{0}\right)$; hence for short times the linear propagator $e^{-i t H}$ can be approximated up to a phase factor by the free particle propagator. In Section 5 we shall see a nonlinear version of this statement.

4.1. An Inverse Strichartz Inequality. Unless indicated otherwise, $0 \in I$ in this section will denote a fixed interval of length at most 1 , and all spacetime norms will be taken over $I \times \mathbf{R}^{d}$.

Suppose $f_{n}$ is a sequence of functions in $\Sigma$ with nontrivial linear evolution $e^{-i t H} f_{n}$. The following refined Strichartz estimate shows that there must be a "frequency" $N_{n}$ which makes a nontrivial contribution to the evolution.

Proposition 4.1 (Refined Strichartz).

$$
\left\|e^{-i t H} f\right\|_{L_{t, x}^{\frac{2(d+2)}{d-2}}} \lesssim\|f\|_{\Sigma}^{\frac{4}{d+2}} \sup _{N}\left\|e^{-i t H} P_{N} f\right\|_{L_{t, x}^{\frac{d-2}{d+2}}}^{\frac{2(d+2)}{d-2}}
$$

Proof. We quote essentially verbatim the proof of Refined Strichartz for the free particle propagator (32 Lemma 3.1). Write $f_{N}$ for $P_{N} f$, where $P_{N}=P_{N}^{H}$ unless 
indicated otherwise. When $d \geq 6$, we apply the square function estimate (2.17), Hölder, Bernstein, and Strichartz to get

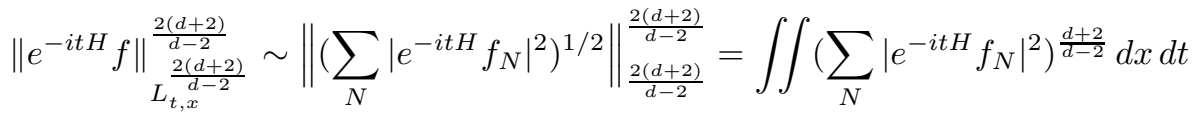

$$
\begin{aligned}
& \lesssim \sum_{M \leq N} \iint\left|e^{-i t H} f_{M}\right|^{\frac{d+2}{d-2}}\left|e^{-i t H} f_{N}\right|^{\frac{d+2}{d-2}} d x d t
\end{aligned}
$$



$$
\begin{aligned}
& \lesssim \sup _{N}\left\|e^{-i t H} f_{N}\right\|_{L_{t, x}^{\frac{2}{d-2}}}^{\frac{L^{2}}{d-2}} \sum_{M \leq N} M^{2}\left\|e^{-i t H} f_{M}\right\|_{L_{t}^{\frac{2(d+2)}{d-4}}} \frac{2 d(d+2)}{L_{x} d^{2}+8}\left\|f_{N}\right\|_{L^{2}} \\
& \lesssim \sup _{N}\left\|e^{-i t H} f_{N}\right\|_{L_{t, x}^{\frac{2(d+2)}{d-2}}}^{\frac{8}{d-2}} \sum_{M \leq N} M^{2}\left\|f_{M}\right\|_{L_{x}^{2}}\left\|f_{N}\right\|_{L_{x}^{2}} \\
& \lesssim \sup _{N}\left\|e^{-i t H} f_{N}\right\|_{\substack{\frac{8}{d-2} \\
L_{t, x}^{\frac{2(d+2)}{d-2}}}} \sum_{M \leq N} \frac{M}{N}\left\|H^{1 / 2} f_{M}\right\|_{L^{2}}\left\|H^{1 / 2} f_{N}\right\|_{L_{x}^{2}} \\
& \lesssim \sup _{N}\left\|e^{-i t H} f_{N}\right\|_{L_{t, x} \frac{\frac{8}{d-2}}{d-2}}^{\frac{L(d+2)}{d-2}}\|f\|_{\Sigma}^{2}
\end{aligned}
$$

The cases $d=3,4,5$ are handled similarly with some minor modifications in the applications of Hölder's inequality.

The next proposition goes one step further and asserts that the sequence $e^{-i t H} f_{n}$ with nontrivial spacetime norm must in fact contain a bubble centered at some $\left(t_{n}, x_{n}\right)$ with spatial scale $N_{n}^{-1}$. We first introduce some vocabulary and notation which will help make the presentation more systematic. Adapting terminology from Ionescu-Pausader-Staffilani [14, we define

Definition 4.1. A frame is a sequence $\left(t_{n}, x_{n}, N_{n}\right) \in I \times \mathbf{R}^{d} \times 2^{\mathbf{N}}$ conforming to one of the following scenarios:

(1) $N_{n} \equiv 1, t_{n} \equiv 0$, and $x_{n} \equiv 0$.

(2) $N_{n} \rightarrow \infty$ and $N_{n}^{-1}\left|x_{n}\right| \rightarrow r_{\infty} \in[0, \infty)$.

Informally, the parameters $t_{n}, x_{n}, N_{n}$ will specify the temporal center, spatial center, and (spatial) frequency of a function. The condition that $\left|x_{n}\right| \lesssim N_{n}$ reflects the fact that we only consider functions obeying some uniform bound in $\Sigma$, and such functions cannot be centered arbitrarily far from the origin. We need to augment the frame $\left\{\left(t_{n}, x_{n}, N_{n}\right)\right\}$ with an auxiliary parameter $N_{n}^{\prime}$, which corresponds to a sequence of spatial cutoffs adapted to the frame.

Definition 4.2. An augmented frame is a sequence $\left(t_{n}, x_{n}, N_{n}, N_{n}^{\prime}\right) \in I \times \mathbf{R}^{d} \times$ $2^{\mathbf{N}} \times \mathbf{R}$ belonging to one of the following types:

(1) $N_{n} \equiv 1, t_{n} \equiv 0, x_{n} \equiv 0, N_{n}^{\prime} \equiv 1$.

(2) $N_{n} \rightarrow \infty, N_{n}^{-1}\left|x_{n}\right| \rightarrow r_{\infty} \in[0, \infty)$, and either

(2a) $N_{n}^{\prime} \equiv 1$ if $r_{\infty}>0$, or

(2b) $N_{n}^{1 / 2} \leq N_{n}^{\prime} \leq N_{n}, N_{n}^{-1}\left|x_{n}\right|\left(\frac{N_{n}}{N_{n}^{\prime}}\right) \rightarrow 0$, and $\frac{N_{n}}{N_{n}^{\prime}} \rightarrow \infty$ if $r_{\infty}=0$. 
Given an augmented frame $\left(t_{n}, x_{n}, N_{n}, N_{n}^{\prime}\right)$, we define scaling and translation operators on functions of space and of spacetime by

$$
\begin{aligned}
\left(G_{n} \phi\right)(x) & =N_{n}^{\frac{d-2}{2}} \phi\left(N_{n}\left(x-x_{n}\right)\right) \\
\left(\tilde{G}_{n} f\right)(t, x) & =N_{n}^{\frac{d-2}{2}} f\left(N_{n}^{2}\left(t-t_{n}\right), N_{n}\left(x-x_{n}\right)\right) .
\end{aligned}
$$

We also define spatial cutoff operators $S_{n}$ by

$$
S_{n} \phi=\left\{\begin{array}{cc}
\phi, & \text { for frames of type } \left.1 \text { (i.e. } N_{n} \equiv 1\right), \\
\chi\left(\frac{N_{n}}{N_{n}^{\prime}}\right) \phi, & \text { for frames of type } \left.2 \quad \text { (i.e. } N_{n} \rightarrow \infty\right),
\end{array}\right.
$$

where $\chi$ is a smooth compactly supported function equal to 1 on the ball $\{|x| \leq 1\}$. An easy computation yields the following mapping properties of these operators:

$$
\begin{array}{r}
\lim _{n \rightarrow \infty} S_{n}=I \text { strongly in } \dot{H}^{1} \text { and in } \Sigma, \\
\limsup _{n \rightarrow \infty}\left\|G_{n}\right\|_{\Sigma \rightarrow \Sigma}<\infty .
\end{array}
$$

For future reference, we record a technical lemma that, as a special case, asserts that the $\Sigma$ norm is controlled almost entirely by the $\dot{H}^{1}$ norm for functions concentrating near the origin.

Lemma 4.2 (Approximation). Let $(q, r)$ be an admissible pair of exponents with $2 \leq r<d$, and let $\mathcal{F}=\left\{\left(t_{n}, x_{n}, N_{n}, N_{n}^{\prime}\right)\right\}$ be an augmented frame of type 2.

(1) Suppose $\mathcal{F}$ is of type 2a in Definition 4.2. Then for $\left\{f_{n}\right\} \subseteq L_{t}^{q} H_{x}^{1, r}\left(\mathbf{R} \times \mathbf{R}^{d}\right)$, we have

$$
\limsup _{n}\left\|\tilde{G}_{n} S_{n} f_{n}\right\|_{L_{t}^{q} \Sigma_{x}^{r}} \lesssim \limsup _{n}\left\|f_{n}\right\|_{L_{t}^{q} H_{x}^{1, r}} .
$$

(2) Suppose $\mathcal{F}$ is of type 20 and $f_{n} \in L_{t}^{q} \dot{H}_{x}^{1, r}\left(\mathbf{R} \times \mathbf{R}^{d}\right)$. Then

$$
\limsup _{n}\left\|\tilde{G}_{n} S_{n} f_{n}\right\|_{L_{t}^{q} \Sigma_{x}^{r}} \lesssim \limsup _{n}\left\|f_{n}\right\|_{L_{t}^{q} \dot{H}_{x}^{1, r}} .
$$

Here $H^{1, r}\left(\mathbf{R}^{d}\right)$ and $\dot{H}^{1, r}\left(\mathbf{R}^{d}\right)$ denote the inhomogeneous and homogeneous $L^{r}$ Sobolev spaces, respectively, equipped with the norms

$$
\|f\|_{H^{1, r}}=\|\langle\nabla\rangle\|_{L^{r}\left(\mathbf{R}^{d}\right)}, \quad\|f\|_{\dot{H}^{1, r}}=\||\nabla| f\|_{L^{r}\left(\mathbf{R}^{d}\right)} .
$$

Proof. By time translation invariance we may assume $t_{n} \equiv 0$. Using Lemma 2.2 we see that it suffices to bound $\left\|\nabla \tilde{G}_{n} S_{n} f_{n}\right\|_{L_{t}^{q} L_{x}^{r}}$ and $\left\||x| \tilde{G}_{n} S_{n} f_{n}\right\|_{L_{t}^{q} L_{x}^{r}}$ separately. By a change of variables, the admissibility condition on $(q, r)$, Hölder, and Sobolev embedding (which necessitates the restriction $r<d$ ), we have

$$
\begin{aligned}
& \left\|\nabla \tilde{G}_{n} S_{n} f_{n}\right\|_{L_{t}^{q} L_{x}^{r}}=\left\|\nabla\left[N_{n}^{\frac{d-2}{2}} f_{n}\left(N_{n}^{2} t, N_{n}\left(x-x_{n}\right)\right) \chi\left(N_{n}^{\prime}\left(x-x_{n}\right)\right)\right]\right\|_{L_{t}^{q} L_{x}^{r}} \\
& \lesssim\left\|\left(\nabla f_{n}\right)(t, x)\right\|_{L_{t}^{q} L_{x}^{r}}+\frac{N_{n}^{\prime}}{N_{n}}\left\|f_{n}(t, x)\right\|_{L_{t}^{q} L_{x}^{r}\left(\mathbf{R} \times\left\{|x| \sim \frac{N_{n}}{N_{n}^{r}}\right\}\right)} \\
& \lesssim\left\|\nabla f_{n}\right\|_{L_{t}^{q} \dot{H}_{x}^{1, r} .}
\end{aligned}
$$

To estimate $\left\||x| \tilde{G}_{n} S_{n} f_{n}\right\|_{L_{t}^{q} L_{x}^{r}}$ we distinguish the two cases. Consider first the case in which $f_{n} \in L_{t}^{q} H_{x}^{1, r}$. Using the bound $\left|x_{n}\right| \lesssim N_{n}$ and a change of variables, we obtain

$$
\left\||x| \tilde{G}_{n} S_{n} f_{n}\right\|_{L_{t}^{q} L^{r}} \lesssim N_{n}^{\frac{d}{2}}\left\|f_{n}\left(N_{n}^{2} t, N_{n}\left(x-x_{n}\right)\right)\right\|_{L^{r}} \lesssim\left\|f_{n}\right\|_{L_{t}^{q} L^{r}} \lesssim\left\|f_{n}\right\|_{L_{t}^{q} H_{x}^{1, r}}
$$


Now consider the second case where $f_{n}$ are merely assumed to lie in $L_{t}^{q} \dot{H}_{x}^{1, r}$. For each $t$, we use Hölder and Sobolev embedding to get

$$
\begin{aligned}
& \left\||x| \tilde{G}_{n} S_{n} f_{n}\right\|_{L_{x}^{r}}^{r}=N_{n}^{\frac{d r}{2}-d-r} \int_{|x| \lesssim \frac{N_{n}}{N_{n}^{\prime}}}\left|x_{n}+N_{n}^{-1} x\right|^{r}\left|f_{n}\left(N_{n}^{2} t, x\right)\right|^{r} d x \\
& \lesssim N_{n}^{\frac{d r}{2}-d}\left[N_{n}^{-r}\left|x_{n}\right|^{r}+N_{n}^{-2 r}\left(\frac{N_{n}}{N_{n}^{\prime}}\right)^{r}\right] \int_{|x| \lesssim \frac{N_{n}}{N_{n}^{\prime}}}\left|f_{n}\left(N_{n}^{2} t, x\right)\right|^{r} d x \\
& \lesssim N_{n}^{\frac{d r}{2}-d}\left[N_{n}^{-r}\left|x_{n}\right|^{r}\left(\frac{N_{n}}{N_{n}^{\prime}}\right)^{r}+\left(N_{n}^{\prime}\right)^{-2 r}\right]\left\|\nabla f_{n}\left(N_{n}^{2} t\right)\right\|_{L_{x}^{r}}^{r} .
\end{aligned}
$$

By the hypotheses on the parameter $N_{n}^{\prime}$ in Definition 4.2, the expression inside the brackets goes to 0 as $n \rightarrow \infty$. After integrating in $t$ and performing a change of variables, we conclude

$$
\left\||x| \tilde{G}_{n} S_{n} f_{n}\right\|_{L_{t}^{q} L_{x}^{r}} \lesssim c_{n}\left\|f_{n}\right\|_{L_{t}^{q} \dot{H}_{x}^{1, r}}
$$

where $c_{n}=o(1)$ as $n \rightarrow \infty$. This completes the proof of the lemma.

Proposition 4.3 (Inverse Strichartz). Let I be a compact interval containing 0 of length at most 1 , and suppose $f_{n}$ is a sequence of functions in $\Sigma\left(\mathbf{R}^{d}\right)$ satisfying

$$
0<\varepsilon \leq\left\|e^{-i t H} f_{n}\right\|_{L_{t, x}^{\frac{2(d+2)}{d-2}}\left(I \times \mathbf{R}^{d}\right)} \lesssim\left\|f_{n}\right\|_{\Sigma} \leq A<\infty .
$$

Then, after passing to a subsequence, there exists an augmented frame

$$
\mathcal{F}=\left\{\left(t_{n}, x_{n}, N_{n}, N_{n}^{\prime}\right)\right\}
$$

and a sequence of functions $\phi_{n} \in \Sigma$ such that one of the following holds:

(1) $\mathcal{F}$ is of type 1 (i.e. $N_{n} \equiv 1$ ) and $\phi_{n}=\phi$ where $\phi \in \Sigma$ is a weak limit of $f_{n}$ in $\Sigma$.

(2) $\mathcal{F}$ is of type Q , either $t_{n} \equiv 0$ or $N_{n}^{2} t_{n} \rightarrow \pm \infty$, and $\phi_{n}=e^{i t_{n} H} G_{n} S_{n} \phi$ where $\phi \in \dot{H}^{1}\left(\mathbf{R}^{d}\right)$ is a weak limit of $G_{n}^{-1} e^{-i t_{n} H} f_{n}$ in $\dot{H}^{1}$. Moreover, if $\mathcal{F}$ is of type [2a, then $\phi$ also belongs to $L^{2}\left(\mathbf{R}^{d}\right)$.

The functions $\phi_{n}$ have the following properties:

$$
\begin{gathered}
\liminf _{n}\left\|\phi_{n}\right\|_{\Sigma} \gtrsim A\left(\frac{\varepsilon}{A}\right)^{\frac{d(d+2)}{8}} \\
\lim _{n \rightarrow \infty}\left\|f_{n}\right\|_{\frac{2 d}{d-2}}^{\frac{2 d}{d-2}}-\left\|f_{n}-\phi_{n}\right\|_{\frac{2 d}{d-2}}^{\frac{2 d}{d-2}}-\left\|\phi_{n}\right\|_{\frac{2 d}{d-2}}^{\frac{2 d}{d-2}}=0 . \\
\lim _{n \rightarrow \infty}\left\|f_{n}\right\|_{\Sigma}^{2}-\left\|f_{n}-\phi_{n}\right\|_{\Sigma}^{2}-\left\|\phi_{n}\right\|_{\Sigma}^{2}=0
\end{gathered}
$$

Proof. The proof will occur in several stages. First we identify the parameters $t_{n}, x_{n}, N_{n}$, which define the location of the bubble $\phi_{n}$ and its characteristic size, and quickly dispose of the case where $N_{n} \equiv 1$. The treatment of the case where $N_{n} \rightarrow \infty$ will be more involved, and we proceed in two steps. We define the profile $\phi_{n}$ and verify the assertions (4.4) and (4.6). Passing to a subsequence, we may assume that the sequence $N_{n}^{2} t_{n}$ converges in $[-\infty, \infty]$. If the limit is infinite, decoupling (4.5) in the $L^{\frac{2 d}{d-2}}$ norm will also follow.

After a brief interlude in which we study certain operator limits, we finish the case where the sequence $N_{n}^{2} t_{n}$ tends to a finite limit. We show that the time parameter $t_{n}$ can actually be redefined to be identically zero after making a negligible correction to the profile $\phi_{n}$, and verify in Lemma 4.13 that the modified profile 
satisfies property (4.5) in addition to (4.4) and (4.6). One can show a posteriori that the original profile $\phi_{n}$ also obeys this last decoupling condition.

By Proposition 4.1, there exist frequencies $N_{n}$ such that

$$
\left\|P_{N_{n}} e^{-i t H} f_{n}\right\|_{L_{t, x}^{\frac{2(d+2)}{d-2}}} \gtrsim \varepsilon^{\frac{d+2}{4}} A^{-\frac{d-2}{4}} .
$$

The comparison of Littlewood-Paley projectors (2.11) implies

$$
\left\|\tilde{P}_{N_{n}} e^{-i t H} f_{n}\right\|_{L_{t, x}^{\frac{2(d+2)}{d-2}}} \gtrsim \varepsilon^{\frac{d+2}{4}} A^{-\frac{d-2}{4}}
$$

where $\tilde{P}_{N}=e^{-H / N^{2}}-e^{-4 H / N^{2}}$ denote the projections based on the heat kernel. By Hölder, Strichartz, and Bernstein,

$$
\begin{aligned}
\varepsilon^{\frac{d+2}{4}} A^{-\frac{d-2}{4}} & \lesssim\left\|\tilde{P}_{N_{n}} e^{-i t H} f_{n}\right\|_{L_{t, x}^{\frac{2(d+2)}{d-2}}} \lesssim\left\|\tilde{P}_{N_{n}} e^{-i t H} f_{n}\right\|_{L_{t, x}^{\frac{d(d+2)}{d}}}^{\frac{d-2}{d}}\left\|\tilde{P}_{N_{n}} e^{-i t H} f_{n}\right\|_{L_{t, x}^{\infty}}^{\frac{2}{d}} \\
& \lesssim\left(N_{n}^{-1} A\right)^{\frac{d-2}{d}}\left\|\tilde{P}_{N_{n}} e^{-i t H} f_{n}\right\|_{L_{t, x}^{\infty}}^{\frac{2}{d}} .
\end{aligned}
$$

Therefore, there exist $\left(t_{n}, x_{n}\right) \in I \times \mathbf{R}^{d}$ such that

$$
\left|e^{-i t_{n} H} \tilde{P}_{N_{n}} f_{n}\left(x_{n}\right)\right| \gtrsim N_{n}^{\frac{d-2}{2}} A\left(\frac{\varepsilon}{A}\right)^{\frac{d(d+2)}{8}} .
$$

The parameters $t_{n}, x_{n}, N_{n}$ will determine the center and width of a bubble. We observe first that the boundedness of $f_{n}$ in $\Sigma$ limits how far the bubble can live from the spatial origin.

Lemma 4.4. We have

$$
\left|x_{n}\right| \leq C_{A, \varepsilon} N_{n}
$$

Proof. Put $g_{n}=\left|e^{-i t_{n} H} f_{n}\right|$. By the kernel bound (2.10),

$$
N_{n^{\frac{d-2}{2}}} A\left(\frac{\varepsilon}{A}\right)^{\frac{d(d+2)}{8}} \lesssim\left|\tilde{P}_{N_{n}} e^{-i t_{n} H} f_{n}\left(x_{n}\right)\right| \lesssim \tilde{P}_{\leq N_{n}}^{\Delta} g_{n}\left(x_{n}\right)+\tilde{P}_{\leq N_{n} / 2}^{\Delta} g_{n}\left(x_{n}\right) .
$$

Thus one of the terms on the right side is at least half as large as the left side, and we only consider the case when

$$
\tilde{P}_{\leq N_{n}}^{\Delta} g_{n}\left(x_{n}\right) \gtrsim N_{n}^{\frac{d-2}{2}} A\left(\frac{\varepsilon}{A}\right)^{\frac{d(d+2)}{8}}
$$

since the argument with $N_{n}$ replaced by $N_{n} / 2$ differs only cosmetically. Informally, $\tilde{P}_{\leq N_{n}}^{\Delta} g_{n}$ is essentially constant over length scales of order $N_{n}^{-1}$, so if it is large at a point $x_{n}$ then it is large on the ball $\left|x-x_{n}\right| \leq N_{n}^{-1}$. More precisely, when $\left|x-x_{n}\right| \leq N_{n}^{-1}$ we have

$$
\begin{aligned}
\tilde{P}_{\leq N_{n} / 2}^{\Delta} g_{n}(x) & =\frac{N_{n}^{d}}{2^{d}(2 \pi)^{\frac{d}{2}}} \int g_{n}(x-y) e^{-\frac{N_{n}^{2}|y|^{2}}{8}} d y \\
& =\frac{N_{n}^{d}}{2^{d}(4 \pi)^{\frac{d}{2}}} \int g_{n}\left(x_{n}-y\right) e^{-\frac{N_{n}^{2}\left|y+x-x_{n}\right|^{2}}{8}} d y \\
& \geq e^{-1} \frac{N_{n}^{d}}{2^{d}(4 \pi)^{\frac{d}{2}}} \int g_{n}\left(x_{n}-y\right) e^{-\frac{N_{n}^{2}|y|^{2}}{2}} d y=e^{-1} 2^{-d} \tilde{P}_{\leq N_{n}}^{\Delta} g_{n}\left(x_{n}\right) \\
& \gtrsim N_{n}^{\frac{d-2}{2}} A\left(\frac{\varepsilon}{A}\right)^{\frac{d(d+2)}{8}} .
\end{aligned}
$$

On the other hand, the mapping properties of the heat kernel imply that

$$
\left\|\tilde{P}_{\leq N_{n} / 2}^{\Delta} g_{n}\right\|_{\Sigma} \lesssim\left(1+N_{n}^{-2}\right) A
$$


Thus,

$$
A \gtrsim\left\|\tilde{P}_{\leq N_{n} / 2}^{\Delta} g_{n}\right\|_{\Sigma} \gtrsim\left\|x \tilde{P}_{\leq N_{n} / 2}^{\Delta} g_{n}\right\|_{L^{2}\left(\left|x-x_{n}\right| \leq N_{n}^{-1}\right)} \gtrsim\left|x_{n}\right| N_{n}^{-\frac{d}{2}} N_{n}^{\frac{d-2}{2}} A\left(\frac{\varepsilon}{A}\right)^{\frac{d(d+2)}{8}},
$$

which yields the claim.

Case 1. Suppose the $N_{n}$ have a bounded subsequence, so that (passing to a subsequence) $N_{n} \equiv N_{\infty}$. The $x_{n}$ 's stay bounded by 4.4, so after passing to a subsequence we may assume $x_{n} \rightarrow x_{\infty}$. We may also assume $t_{n} \rightarrow t_{\infty}$ since the interval $I$ is compact. The functions $f_{n}$ are bounded in $\Sigma$, hence (after passing to a subsequence) converge weakly in $\Sigma$ to a function $\phi$.

We show that $\phi$ is nontrivial in $\Sigma$. Indeed,

$$
\begin{aligned}
\left\langle\phi, e^{i t_{\infty} H} \tilde{P}_{N_{\infty}} \delta_{x_{\infty}}\right\rangle & =\lim _{n}\left\langle f_{n}, e^{i t_{\infty} H} \tilde{P}_{N_{\infty}} \delta_{x_{\infty}}\right\rangle \\
& =\lim _{n \rightarrow \infty}\left[e^{-i t_{n} H} \tilde{P}_{N_{\infty}} f_{n}\left(x_{n}\right)+\left\langle f_{n},\left(e^{i t_{\infty} H}-e^{i t_{n} H}\right) \tilde{P}_{N_{\infty}} \delta_{x_{n}}\right\rangle\right. \\
& \left.+\left\langle f_{n}, e^{i t_{\infty} H} \tilde{P}_{N_{n}}\left(\delta_{x_{\infty}}-\delta_{x_{n}}\right)\right\rangle\right] .
\end{aligned}
$$

Using the heat kernel bounds (2.10) and the fact that, by the compactness of the embedding $\Sigma \subset L^{2}$, the sequence $f_{n}$ converges to $\phi$ in $L^{2}$, one verifies easily that the second and third terms on the right side vanish. So

$$
\left|\left\langle\phi, e^{i t_{\infty} H} \tilde{P}_{N_{\infty}} \delta_{x_{\infty}}\right\rangle\right|=\lim _{n \rightarrow \infty}\left|e^{-i t_{n} H} \tilde{P}_{N_{\infty}} f_{n}\left(x_{n}\right)\right| \gtrsim N_{\infty}^{\frac{d-2}{2}} \varepsilon^{\frac{d(d+2)}{8}} A^{-\frac{(d-2)(d+4)}{8}} .
$$

On the other hand, by Hölder and (2.10),

$$
\begin{aligned}
\left|\left\langle\phi, e^{i t_{\infty} H} \tilde{P}_{N_{\infty}} \delta_{x_{\infty}}\right\rangle\right| & \leq\left\|e^{-i t_{\infty} H} \phi\right\|_{L^{\frac{2 d}{d-2}}}\left\|\tilde{P}_{N_{\infty}} \delta_{x_{\infty}}\right\|_{L^{\frac{2 d}{d+2}}} \\
& \lesssim\|\phi\|_{\Sigma} N_{\infty}^{\frac{d-2}{2}} .
\end{aligned}
$$

Therefore

$$
\|\phi\|_{\Sigma} \gtrsim \varepsilon^{\frac{d(d+2)}{8}} A^{-\frac{(d-2)(d+4)}{8}} .
$$

Set

$$
\phi_{n} \equiv \phi,
$$

and define the augmented frame $\left(t_{n}, x_{n}, N_{n}, N_{n}^{\prime}\right) \equiv(0,0,1,1)$. The decoupling in $\Sigma$ (4.6) can be proved as in Case 2 below, and we refer the reader to the argument detailed there. It remains to establish decoupling in $L^{\frac{2 d}{d-2}}$. As the embedding $\Sigma \subset L^{2}$ is compact, the sequence $f_{n}$, which converges weakly to $\phi \in \Sigma$, converges to $\phi$ strongly in $L^{2}$. After passing to a subsequence we obtain convergence pointwise a.e. The decoupling (4.5) now follows from Lemma 2.6. This completes the case where $N_{n}$ have a bounded subsequence.

Case 2. We now address the case where $N_{n} \rightarrow \infty$. The main nuisance is that the weak limits $\phi$ will usually be merely in $\dot{H}^{1}\left(\mathbf{R}^{d}\right)$, not in $\Sigma$, so defining the profiles $\phi_{n}$ will require spatial cutoffs.

As the functions $N_{n}^{-(d-2) / 2}\left(e^{-i t_{n} H} f_{n}\right)\left(N_{n}^{-1} \cdot+x_{n}\right)$ are bounded in $\dot{H}^{1}\left(\mathbf{R}^{d}\right)$, the sequence has a weak subsequential limit

$$
N_{n}^{-\frac{d-2}{2}}\left(e^{-i t_{n} H} f_{n}\right)\left(N_{n}^{-1} \cdot+x_{n}\right) \rightarrow \phi \text { in } \dot{H}^{1}\left(\mathbf{R}^{d}\right) .
$$

By Lemma 4.4 after passing to a further subsequence we may assume

$$
\lim _{n \rightarrow \infty} N_{n}^{-1}\left|x_{n}\right|=r_{\infty}<\infty \text { and } \lim _{n \rightarrow \infty} N_{n}^{2} t_{n}=t_{\infty} \in[-\infty, \infty] .
$$


It will be necessary to distinguish the cases $r_{\infty}>0$ and $r_{\infty}=0$, corresponding to whether the frame $\left\{\left(t_{n}, x_{n}, N_{n}\right)\right\}$ is type $2 \mathrm{a}$ or $2 \mathrm{~b}$, respectively.

Lemma 4.5. If $r_{\infty}>0$, the function $\phi$ defined in (4.8) also belongs to $L^{2}$.

Proof. By (4.8) and the Rellich-Kondrashov compactness theorem, for each $R \geq 1$ we have

$$
N_{n}^{-\frac{d-2}{2}}\left(e^{-i t_{n} H} f_{n}\right)\left(N_{n}^{-1} \cdot+x_{n}\right) \rightarrow \phi \text { in } L^{2}(\{|x| \leq R\}) .
$$

By a change of variables,

$$
\begin{aligned}
N_{n}^{-\frac{d-2}{2}}\left(e^{-i t_{n} H} f_{n}\right)\left(N_{n}^{-1} \cdot+x_{n}\right) \|_{L^{2}(|x| \leq R)} & =N_{n}\left\|e^{-i t_{n} H} f_{n}\right\|_{L^{2}\left(\left|x-x_{n}\right| \leq R N_{n}^{-1}\right)} \\
& \lesssim\left\|x e^{-i t_{n} H} f_{n}\right\|_{L^{2}}
\end{aligned}
$$

whenever $\left|x_{n}\right| \geq \frac{N_{n} r_{\infty}}{2}$ and $R N_{n}^{-1} \leq \frac{r_{\infty}}{10}$, so we have uniformly in $R \geq 1$ that

$$
\limsup _{n}\left\|N_{n}^{-\frac{d-2}{2}}\left(e^{-i t_{n} H} f_{n}\right)\left(N_{n}^{-1} \cdot+x_{n}\right)\right\|_{L^{2}(|x| \leq R)} \lesssim \sup _{n}\left\|e^{-i t_{n} H} f_{n}\right\|_{\Sigma} \lesssim 1
$$

Therefore $\|\phi\|_{L^{2}}=\lim _{R \rightarrow \infty}\|\phi\|_{L^{2}(|x| \leq R)} \lesssim 1$.

Remark. The claim fails if $r_{\infty}=0$. Indeed, if $\phi \in \dot{H}^{1}\left(\mathbf{R}^{d}\right) \backslash L^{2}\left(\mathbf{R}^{d}\right)$, then $f_{n}=N_{n}^{(d-2) / 2} \phi\left(N_{n} \cdot\right) \chi(\cdot)$ are bounded in $\Sigma$, and $N_{n}^{-(d-2) / 2} f_{n}\left(N_{n}^{-1} \cdot\right)=\phi(\cdot) \chi\left(N_{n}^{-1} \cdot\right)$ converges strongly in $\dot{H}^{1}$ to $\phi$.

Next we prove that $\phi$ is nontrivial in $\dot{H}^{1}$.

Lemma 4.6. $\|\phi\|_{\dot{H}^{1}} \gtrsim A\left(\frac{\varepsilon}{A}\right)^{\frac{d(d+2)}{8}}$.

Proof. From (2.10) and (4.7),

$$
N_{n}^{\frac{d-2}{2}} A\left(\frac{\varepsilon}{A}\right)^{\frac{d(d+2)}{8}} \lesssim \tilde{P}_{\leq N_{n}}^{\Delta}\left|e^{-i t_{n} H} f_{n}\right|\left(x_{n}\right)+\tilde{P}_{\leq N_{n} / 2}^{\Delta}\left|e^{-i t_{n} H} f_{n}\right|\left(x_{n}\right),
$$

so one of the terms on the right is at least half the left side. Suppose first that

$$
\tilde{P}_{\leq N_{n}}^{\Delta}\left|e^{-i t_{n} H} f_{n}\right|\left(x_{n}\right) \gtrsim N_{n}^{\frac{d-2}{2}} A\left(\frac{\varepsilon}{A}\right)^{\frac{d(d+2)}{8}} .
$$

Put $\check{\psi}=\tilde{P}_{\leq 1}^{\Delta} \delta_{0}=e^{\Delta} \delta_{0}$. Since $\check{\psi}$ is Schwartz,

$$
\left|\langle|\phi|, \breve{\psi}\rangle_{L^{2}}\right| \leq\|\phi\|_{\dot{H}^{1}}\|\check{\psi}\|_{\dot{H}^{-1}} \lesssim\|\phi\|_{\dot{H}^{1}} .
$$

On the other hand, as the absolute values $N_{n}^{-\frac{d-2}{2}}\left|e^{-i t_{n} H} f_{n}\right|\left(N_{n}^{-1} \cdot+x_{n}\right)$ converge weakly in $\dot{H}^{1}$ to $|\phi|$, we have

$$
\begin{aligned}
\langle|\phi|, \check{\psi}\rangle_{L^{2}} & =\lim _{n}\left\langle N_{n}^{-\frac{d-2}{2}}\left|e^{-i t_{n} H} f_{n}\right|\left(N_{n}^{-1} \cdot+x_{n}\right), \check{\psi}\right\rangle_{L^{2}} \\
& =\lim _{n} \tilde{P}_{\leq N_{n}}^{\Delta}\left|e^{-i t_{n} H} f_{n}\right|\left(x_{n}\right) \gtrsim A\left(\frac{\varepsilon}{A}\right)^{\frac{d(d+2)}{8}} .
\end{aligned}
$$

from which the claim follows. Similarly if

$$
\tilde{P}_{\leq N_{n} / 2}^{\Delta}\left|e^{-i t_{n} H} f_{n}\right|\left(x_{n}\right) \gtrsim N_{n}^{\frac{d-2}{2}} A\left(\frac{\varepsilon}{A}\right)^{\frac{d(d+2)}{8}},
$$

then we obtain $\|\phi\|_{\dot{H}^{1}} \sim\|\phi(2 \cdot)\|_{\dot{H}^{1}} \gtrsim N_{n}^{\frac{d-2}{2}} A\left(\frac{\varepsilon}{A}\right)^{\frac{d(d+2)}{8}}$. 
Having extracted a nontrivial bubble $\phi$, we are ready to define the $\phi_{n}$. The basic idea is to undo the operations applied to $f_{n}$ in the definition (4.8) of $\phi$. However, we need to first apply a spatial cutoff to embed $\phi$ in $\Sigma$.

With the frame $\left\{\left(t_{n}, x_{n}, N_{n}\right)\right\}$ defined according to (4.7), we form the augmented frame $\left\{\left(t_{n}, x_{n}, N_{n}, N_{n}^{\prime}\right)\right\}$ with the cutoff parameter $N_{n}^{\prime}$ chosen according to the second case of Definition 4.2. Let $G_{n}, S_{n}$ be the $\dot{H}^{1}$ isometries and spatial cutoff operators associated to $\left\{\left(t_{n}, x_{n}, N_{n}, N_{n}^{\prime}\right)\right\}$. Set

$$
\phi_{n}=e^{i t_{n} H} G_{n} S_{n} \phi=e^{i t_{n} H}\left[N_{n}^{\frac{d-2}{2}} \phi\left(N_{n}\left(\cdot-x_{n}\right)\right) \chi\left(N_{n}^{\prime}\left(\cdot-x_{n}\right)\right)\right] .
$$

We now verify that the $\phi_{n}$ satisfy the various properties claimed in the proposition.

Lemma 4.7. $A\left(\frac{\varepsilon}{A}\right)^{\frac{d(d+2)}{8}} \lesssim \liminf _{n \rightarrow \infty}\left\|\phi_{n}\right\|_{\Sigma} \leq \limsup _{n \rightarrow \infty}\left\|\phi_{n}\right\|_{\Sigma} \lesssim 1$.

Proof. By the definition of the $\Sigma$ norm and a change of variables,

$$
\left\|\phi_{n}\right\|_{\Sigma}=\left\|G_{n} S_{n}\right\|_{\Sigma} \geq\left\|S_{n} \phi\right\|_{\dot{H}^{1}} .
$$

Hence Lemma 4.6 and the remarks following Definition 4.2 together imply the lower bound

$$
\liminf _{n}\left\|\phi_{n}\right\|_{\Sigma} \gtrsim A\left(\frac{\varepsilon}{A}\right)^{\frac{d(d+2)}{8}} .
$$

The upper bound follows immediately from the case $(q, r)=(\infty, 2)$ in Lemma 4.2

We verify the decoupling property (4.6). By the Pythagorean theorem,

$$
\begin{aligned}
\left\|f_{n}\right\|_{\Sigma}^{2}-\left\|f_{n}-\phi_{n}\right\|_{\Sigma}^{2}-\left\|\phi_{n}\right\|_{\Sigma}^{2} & =2 \operatorname{Re}\left(\left\langle f_{n}-\phi_{n}, \phi_{n}\right\rangle_{\Sigma}\right) \\
& =2 \operatorname{Re}\left(\left\langlee^{-i t_{n} H} f_{n}-G_{n} S_{n} \phi, G_{n} S_{n}\right.\right. \\
& =2 \operatorname{Re}\left(\left\langle w_{n}, G_{n} S_{n} \phi\right\rangle_{\Sigma}\right) .
\end{aligned}
$$

where $w_{n}=e^{-i t_{n} H} f_{n}-G_{n} S_{n} \phi$. By definition,

$$
\left\langle w_{n}, G_{n} S_{n} \phi\right\rangle_{\Sigma}=\left\langle w_{n}, G_{n} S_{n} \phi\right\rangle_{\dot{H}^{1}}+\left\langle x w_{n}, x G_{n} S_{n} \phi\right\rangle_{L^{2}} .
$$

From (4.3) and the definition (4.8) of $\phi$, it follows that

$$
G_{n}^{-1} w_{n} \rightarrow 0 \quad \text { weakly in } \quad \dot{H}^{1} \quad \text { as } n \rightarrow \infty .
$$

Hence

$$
\lim _{n \rightarrow \infty}\left\langle w_{n}, G_{n} S_{n} \phi\right\rangle_{\dot{H}^{1}}=\lim _{n \rightarrow \infty}\left\langle G_{n}^{-1} w_{n}, S_{n} \phi\right\rangle_{\dot{H}^{1}}=\lim _{n \rightarrow \infty}\left\langle G_{n}^{-1} w_{n}, \phi\right\rangle_{\dot{H}^{1}}=0 .
$$

We turn to the second component of the inner product. Fix $R>0$, and estimate

$$
\begin{aligned}
& \left|\left\langle x w_{n}, x G_{n} S_{n} \phi\right\rangle_{L^{2}}\right| \\
& \leq \int_{\left\{\left|x-x_{n}\right| \leq R N_{n}^{-1}\right\}}\left|x w_{n}\right|\left|x G_{n} S_{n} \phi\right| d x+\int_{\left\{|x-x-n|>R N_{n}^{-1}\right\}}\left|x w_{n}\right|\left|x G_{n} S_{n} \phi\right| d x \\
& =(I)+(I I)
\end{aligned}
$$

Perform a change of variable and drop the spatial cutoff $S_{n}$, keeping in mind the bound $\left|x_{n}\right| \lesssim N_{n}$, to obtain

$$
(I) \lesssim \int_{|x| \leq R}\left|G_{n}^{-1} w_{n}\right||\phi| d x \rightarrow 0 \quad \text { as } \quad n \rightarrow \infty .
$$


Next, apply Cauchy-Schwartz and the upper bound of Lemma 4.7 to see that

$$
\begin{aligned}
& (I I)^{2} \lesssim \int_{\left\{\left|x-x_{n}\right|>R N_{n}^{-1}\right\}}\left|x G_{n} S_{n} \phi\right|^{2} d x \\
& \lesssim N_{n}^{-2} \int_{R \leq|x| \lesssim \frac{N_{n}}{N_{n}^{\prime}}}\left|x_{n}+N_{n}^{-1} x\right|^{2}|\phi(x)|^{2} d x \\
& \lesssim\left(N_{n}^{-2}\left|x_{n}\right|^{2}+N_{n}^{-2}\left(N_{n}^{\prime}\right)^{-2}\right) \int_{R \leq|x| \lesssim \frac{N_{n}}{N_{n}^{n}}}|\phi(x)|^{2} d x .
\end{aligned}
$$

Suppose that the frame $\left\{\left(t_{n}, x_{n}, N_{n}\right)\right\}$ is of type 2a, so that $\lim _{n} N_{n}^{-1}\left|x_{n}\right|>0$. By Lemma 4.5 and dominated convergence, the right side above is bounded by

$$
\int_{R \leq|x|}|\phi(x)|^{2} d x \rightarrow 0 \quad \text { as } \quad R \rightarrow \infty
$$

uniformly in $n$. If instead $\left\{\left(t_{n}, x_{n}, N_{n}\right)\right\}$ is of type 2b use Hölder to see that the right side is bounded by

$$
\left(N_{n}^{-2}\left|x_{n}\right|\left(\frac{N_{n}}{N_{n}^{\prime}}\right)^{2}+\left(N_{n}^{\prime}\right)^{-4}\right)\|\phi\|_{L \frac{2 d}{d-2}}
$$

By Sobolev embedding and the construction of the parameter $N_{n}^{\prime}$ in Definition 4.2, the above vanishes as $n \rightarrow \infty$. In either case, we obtain

$$
\lim _{R \rightarrow \infty} \limsup _{n \rightarrow \infty}(I I)=0 \text {. }
$$

Combining the two estimates and choosing $R$ arbitrarily large, we conclude as required that

$$
\lim _{n \rightarrow \infty}\left|\left\langle x w_{n}, x G_{n} S_{n} \phi\right\rangle_{L^{2}}\right|=0 .
$$

To close this subsection, we verify the $L^{\frac{2 d}{d-2}}$ decoupling property 4.5 when $N_{n}^{2} t_{n} \rightarrow \pm \infty$. Assume first that the $\phi$ appearing in the definition (4.10) of $\phi_{n}$ has compact support. By the dispersive estimate (2.5) and a change of variables, we have

$$
\lim _{n \rightarrow \infty}\left\|\phi_{n}\right\|_{L^{\frac{2 d}{d-2}}} \lesssim\left|t_{n}\right|^{-1}\left\|G_{n} \phi\right\|_{L^{\frac{2 d}{d+2}}} \lesssim\left(N_{n}^{2}\left|t_{n}\right|\right)^{-1}\|\phi\|_{L^{\frac{2 d}{d+2}}}=0 .
$$

The claimed decoupling follows immediately.

For general $\phi$ in $H^{1}$ or $\dot{H}^{1}$ (depending on whether $\lim _{n} N_{n}^{-1}\left|x_{n}\right|$ is positive or zero), select $\psi^{\varepsilon} \in C_{c}^{\infty}$ converging to $\phi$ in the appropriate norm as $\varepsilon \rightarrow 0$. Then for all $n$ large enough, we have

$$
\left\|\phi_{n}\right\|_{L^{\frac{2 d}{d-2}}} \leq\left\|e^{i t_{n} H} G_{n} S_{n}\left[\phi-\psi^{\varepsilon}\right]\right\|_{L^{\frac{2 d}{d-2}}}+\left\|e^{i t_{n} H} G_{n} S_{n} \psi^{\varepsilon}\right\|_{L^{\frac{2 d}{d-2}}},
$$

and we once again have decoupling by Lemmas 2.3 and 4.2 , and the special case just proved.

4.2. Convergence of linear propagators. To complete the proof of Proposition 4.3 we need a more detailed understanding of how the linear propagator $e^{-i t H}$ interacts with the $\dot{H}^{1}$-symmetries $G_{n}$ associated to a frame in certain limiting situations. The lemmas proved in this section are heavily inspired by the discussion surrounding [20, Lemma 5.2], in which the authors prove analogous results relating the linear propagators of the 2D Schrödinger equation and the complexified KleinGordon equation $-i v_{t}+\langle\nabla\rangle v=0$. We begin by introducing some terminology due to Ionescu-Pausader-Staffilani [14]. 
Definition 4.3. We say two frames $\mathcal{F}^{1}=\left\{\left(t_{n}^{1}, x_{n}^{1}, N_{n}^{1}\right)\right\}$ and $\mathcal{F}^{2}=\left\{\left(t_{n}^{2}, x_{n}^{2}, N_{n}^{2}\right)\right\}$ (where the superscripts are indices, not exponents) are equivalent if

$$
\frac{N_{n}^{1}}{N_{n}^{2}} \rightarrow R_{\infty} \in(0, \infty), N_{n}^{1}\left(x_{n}^{2}-x_{n}^{1}\right) \rightarrow x_{\infty} \in \mathbf{R}^{d},\left(N_{n}^{1}\right)^{2}\left(t_{n}^{1}-t_{n}^{2}\right) \rightarrow t_{\infty} \in \mathbf{R} .
$$

If any of the above statements fails, we say that $\mathcal{F}_{1}$ and $\mathcal{F}_{2}$ are orthogonal. Note that replacing the $N_{n}^{1}$ in the second and third expressions above by $N_{n}^{2}$ yields an equivalent definition of orthogonality.

Remark. If $\mathcal{F}^{1}$ and $\mathcal{F}^{2}$ are equivalent, it follows from the above definition that they must be of the same type in Definition 4.1, and that $\lim _{n}\left(N_{n}^{1}\right)^{-1}\left|x_{n}^{1}\right|$ and $\lim _{n}\left(N_{n}^{2}\right)^{-1}\left|x_{n}^{2}\right|$ are either both zero or both positive.

One interpretation of the following lemma and its corollary is that when acting on functions concentrated at a point, $e^{-i t H}$ can be approximated for small $t$ by regarding the $|x|^{2} / 2$ potential as essentially constant on the support of the initial data; thus one obtains a modulated free particle propagator $e^{-\frac{i t\left|x_{0}\right|^{2}}{2}} e^{\frac{i t \Delta}{2}}$ where $x_{0}$ is the spatial center of the initial data.

Lemma 4.8 (Strong convergence). Suppose

$$
\mathcal{F}^{M}=\left(t_{n}^{M}, x_{n}, M_{n}\right), \quad \mathcal{F}^{N}=\left(t_{n}^{N}, y_{n}, N_{n}\right)
$$

are equivalent frames. Define

$$
\begin{aligned}
R_{\infty} & =\lim _{n \rightarrow \infty} \frac{M_{n}}{N_{n}}, t_{\infty}=\lim _{n \rightarrow \infty} M_{n}^{2}\left(t_{n}^{M}-t_{n}^{N}\right), x_{\infty}=\lim _{n \rightarrow \infty} M_{n}\left(y_{n}-x_{n}\right) \\
r_{\infty} & =\lim _{n} M_{n}^{-1}\left|x_{n}\right|=\lim _{n} M_{n}^{-1}\left|y_{n}\right| .
\end{aligned}
$$

Let $G_{n}^{M}, G_{n}^{N}$ be the scaling and translation operators attached to the frames $\mathcal{F}^{M}$ and $\mathcal{F}^{N}$ respectively. Then $\left(e^{-i t_{n}^{N} H} G_{n}^{N}\right)^{-1} e^{-i t_{n}^{M} H} G_{n}^{M}$ converges in the strong operator topology on $B(\Sigma, \Sigma)$ to the operator $U_{\infty}$ defined by

$$
U_{\infty} \phi=e^{-\frac{i t_{\infty}\left(r_{\infty}\right)^{2}}{2}} R_{\infty}^{\frac{d-2}{2}}\left[e^{\frac{i t_{\infty} \Delta}{2}} \phi\right]\left(R_{\infty} \cdot+x_{\infty}\right) .
$$

Proof. If $M_{n} \equiv 1$, then by the definition of a frame we must have $\mathcal{F}^{M}=\mathcal{F}^{N}=$ $\{(1,0,0)\}$, so the claim is trivial. Thus we may assume that $M_{n} \rightarrow \infty$. Put $t_{n}=t_{n}^{M}-t_{n}^{N}$. Using Mehler's formula (2.4), we write

$$
\begin{aligned}
& \left(e^{-i t_{n}^{N} H} G_{n}^{N}\right)^{-1} e^{-i t_{n}^{M} H} G_{n}^{M}=\left(G_{n}^{N}\right)^{-1} e^{-i t_{n} H} G_{n}^{M} \phi(x) \\
& =\left(\frac{M_{n}}{N_{n}}\right)^{\frac{d-2}{2}} e^{i \gamma\left(t_{n}\right)\left|y_{n}+N_{n}^{-1} x\right|^{2}} e^{\frac{i M_{n}^{2} \sin \left(t_{n}\right) \Delta}{2}}\left[e^{i \gamma\left(t_{n}\right)\left|x_{n}+M_{n}^{-1} \cdot\right|^{2}} \phi\right]\left(\frac{M_{n}}{N_{n}} x+M_{n}\left(y_{n}-x_{n}\right)\right) .
\end{aligned}
$$

where

We see that

$$
\gamma(t)=\frac{\cos t-1}{2 \sin t}=-\frac{t}{4}+O\left(t^{3}\right)
$$

$$
e^{i \gamma\left(t_{n}\right)\left|x_{n}+M_{n}^{-1} \cdot\right|^{2} \phi \rightarrow e^{-\frac{i t_{\infty}\left(r_{\infty}\right)^{2}}{4} \phi} \quad \text { in } \Sigma .}
$$

Indeed,

$$
\begin{aligned}
& \left\|\nabla\left[e^{i \gamma\left(t_{n}\right)\left|x_{n}+M_{n}^{-1} \cdot\right|^{2}} \phi-e^{i \gamma\left(t_{n}\right)\left|x_{n}\right|^{2}} \phi\right]\right\|_{L^{2}}=\left\|\nabla_{x}\left[\left(e^{i \gamma\left(t_{n}\right)\left[M_{n}^{-2}|x|^{2}+M_{n}^{-1} x_{n} \cdot x\right]}-1\right) \phi\right]\right\|_{L^{2}} \\
& \lesssim\left\|t_{n}\left(M_{n}^{-2} x+M_{n}^{-1} x_{n}\right) \phi\right\|_{L^{2}}+\left\|\left(e^{i \gamma\left(t_{n}\right)\left[M_{n}^{-2}|x|^{2}+2 M_{n}^{-1} x_{n} \cdot x\right]}-1\right) \nabla \phi\right\|_{L^{2}} \\
& \lesssim\left|t_{n}\right| M_{n}^{-2}\|x \phi\|_{L^{2}}+\left|t_{n}\left\|x_{n} \mid M_{n}^{-1}\right\| \phi\left\|_{L^{2}}+\right\|\left(e^{i \gamma\left(t_{n}\right)\left[M_{n}^{-2}|x|^{2} 2 M_{n}^{-1} x_{n} \cdot x\right]}-1\right) \nabla \phi \|_{L^{2}} .\right.
\end{aligned}
$$


As $n \rightarrow \infty$, the first two terms vanish because $\|x \phi\|_{2}+\|\phi\|_{2} \lesssim\|\phi\|_{\Sigma}$, while the third term vanishes by dominated convergence. Dominated convergence also implies that

$$
\left\|x\left[e^{i \gamma\left(t_{n}\right)\left|x_{n}+M_{n}^{-1} x\right|^{2}} \phi-e^{i \gamma\left(t_{n}\right)\left|x_{n}\right|^{2}} \phi\right]\right\|_{L^{2}} \rightarrow 0 \text { as } n \rightarrow \infty .
$$

On the other hand, since

$$
\gamma\left(t_{n}\right)\left|x_{n}\right|^{2}=-\frac{M_{n}^{2} t_{n} M_{n}^{-2}\left|x_{n}\right|^{2}}{4}+O\left(M_{n}^{-4}\right) \rightarrow-\frac{t_{\infty}\left(r_{\infty}\right)^{2}}{4},
$$

it follows that

$$
\left\|e^{i \gamma\left(t_{n}\right)\left|x_{n}+M_{n}^{-1} \cdot\right|^{2}} \phi-e^{-\frac{i t_{\infty}\left(r_{\infty}\right)^{2}}{4}} \phi\right\|_{\Sigma} \rightarrow 0
$$

as claimed. Now, using that $e^{\frac{i M_{n}^{2} \sin \left(t_{n}\right) \Delta}{2}} \rightarrow e^{\frac{i t_{\infty} \Delta}{2}}$ in the strong operator topology on $B(\Sigma, \Sigma)$, we obtain

$$
e^{\frac{i M_{n}^{2} \sin \left(t_{n}\right) \Delta}{2}}\left[e^{i \gamma\left(t_{n}\right)\left|x_{n}+M_{n}^{-1} \cdot\right|^{2}} \phi\right] \rightarrow e^{-\frac{i t_{\infty}\left(r_{\infty}\right)^{2}}{4}} e^{\frac{i t_{\infty} \Delta}{2}} \phi \text { in } \Sigma,
$$

and the full conclusion quickly follows.

Corollary 4.9. Let $\left\{\left(t_{n}^{M}, x_{n}, M_{n}, M_{n}^{\prime}\right)\right\}$ and $\left\{\left(t_{n}^{N}, y_{n}, N_{n}, N_{n}^{\prime}\right)\right\}$ be augmented frames such that $\left\{\left(t_{n}^{M}, x_{n}, M_{n}\right)\right\}$ and $\left\{\left(t_{n}^{N}, y_{n}, N_{n}\right)\right\}$ are equivalent. Let $S_{n}^{M}, S_{n}^{N}$ be the associated spatial cutoff operators as defined in (4.2). Then

$$
\lim _{n \rightarrow \infty}\left\|e^{-i t_{n}^{M} H} G_{n}^{M} S_{n}^{M} \phi-e^{-i t_{n}^{N} H} G_{n}^{N} S_{n}^{N} U_{\infty} \phi\right\|_{\Sigma}=0
$$

and

$$
\lim _{n \rightarrow \infty}\left\|e^{-i t_{n}^{M} H} G_{n}^{M} S_{n}^{M} \phi-e^{-i t_{n}^{N} H} G_{n}^{N} U_{\infty} S_{n}^{N} \phi\right\|_{\Sigma}=0
$$

whenever $\phi \in H^{1}$ if the frames conform to case 2a and $\phi \in \dot{H}^{1}$ if they conform to case 2b in Definition 4.2 .

Proof. As before, the result is immediate if $M_{n} \equiv 1$ since all operators in sight are trivial. Thus we may assume $M_{n} \rightarrow \infty$. Suppose first that $\phi \in C_{c}^{\infty}$. Using the unitarity of $e^{-i t H}$ on $\Sigma$, the operator bounds (4.3), and the fact that $S_{n}^{M} \phi=\phi$ for all $n$ sufficiently large, we write the left side of (4.11) as

$$
\begin{aligned}
& \left\|G_{n}^{N}\left[\left(G_{n}^{N}\right)^{-1} e^{-i\left(t_{n}^{M}-t_{n}^{N}\right) H} G_{n}^{M} \phi-S_{n}^{N} U_{\infty} \phi\right]\right\|_{\Sigma} \\
& \lesssim\left\|\left(G_{n}^{N}\right)^{-1} e^{-i\left(t_{n}^{M}-t_{n}^{N}\right) H} G_{n}^{M} \phi-S_{n}^{N} U_{\infty} \phi\right\|_{\Sigma} \\
& \lesssim\left\|\left(G_{n}^{N}\right)^{-1} e^{-i\left(t_{n}^{M}-t_{n}^{N}\right) H} G_{n}^{M} \phi-U_{\infty} \phi\right\|_{\Sigma}+\left\|\left(1-S_{n}^{N}\right) U_{\infty} \phi\right\|_{\Sigma}
\end{aligned}
$$

which goes to zero by Lemma 4.8 and dominated convergence. This proves (4.11) under the additional hypothesis that $\phi \in C_{c}^{\infty}$.

We now remove this crutch and take $\phi \in H^{1}$ or $\dot{H}^{1}$ depending on whether the frames are of type 2a or 2b in Definition 4.2, respectively. For each $\varepsilon>0$, choose $\phi^{\varepsilon} \in C_{c}^{\infty}$ such that $\left\|\phi-\phi^{\varepsilon}\right\|_{H^{1}}<\varepsilon$ or $\left\|\phi-\phi^{\varepsilon}\right\|_{\dot{H}^{1}}<\varepsilon$, respectively. Then

$$
\begin{aligned}
& \left\|e^{-i t_{n}^{M} H} G_{n}^{M} S_{n}^{M} \phi-e^{-i t_{n}^{N} H} G_{n}^{N} S_{n}^{N} U_{\infty} \phi\right\|_{\Sigma} \leq\left\|e^{-i t_{n}^{M} H} G_{n}^{M} S_{n}^{M}\left(\phi-\phi^{\varepsilon}\right)\right\|_{\Sigma} \\
& +\left\|e^{-i t_{n} H} G_{n}^{M} S_{n}^{M} \phi^{\varepsilon}-e^{-i t_{n}^{N} H} G_{n}^{N} S_{n}^{N} U_{\infty} \phi^{\varepsilon}\right\|_{\Sigma}+\left\|e^{-i t_{n}^{N} H} G_{n}^{N} S_{n}^{N} U_{\infty}\left(\phi-\phi^{\varepsilon}\right)\right\|_{\Sigma}
\end{aligned}
$$

In the limit as $n \rightarrow \infty$, the middle term vanishes and we are left with a quantity at most a constant times

$$
\limsup _{n \rightarrow \infty}\left\|G_{n}^{M} S_{n}^{M}\left(\phi-\phi^{\varepsilon}\right)\right\|_{\Sigma}+\limsup _{n \rightarrow \infty}\left\|G_{n}^{N} S_{n}^{N} U_{\infty}\left(\phi-\phi^{\varepsilon}\right)\right\|_{\Sigma}
$$


Applying Lemma 4.2 and using the mapping properties of $U_{\infty}$ on $\dot{H}^{1}$ and $H^{1}$, we see that

$$
\limsup _{n \rightarrow \infty}\left\|e^{-i t_{n} H} G_{n}^{M} S_{n}^{M} \phi-e^{i t_{n}^{N} H} G_{n}^{N} S_{n}^{N} U_{\infty} \phi\right\|_{\Sigma} \lesssim \varepsilon
$$

for every $\varepsilon>0$. This proves the claim (4.11). Similar considerations handle the second claim (4.12).

Lemma 4.10. Suppose the frames $\left\{\left(t_{n}^{M}, x_{n}, M_{n}\right)\right\}$ and $\left\{\left(t_{n}^{N}, y_{n}, N_{n}\right)\right\}$ are equivalent. Put $t_{n}=t_{n}^{M}-t_{n}^{N}$. Then for $f, g \in \Sigma$ we have

$$
\left\langle\left(G_{n}^{N}\right)^{-1} e^{-i t_{n} H} G_{n}^{M} f, g\right\rangle_{\dot{H}^{1}}=\left\langle f,\left(G_{n}^{M}\right)^{-1} e^{i t_{n} H} G_{n}^{N} g\right\rangle_{\dot{H}^{1}}+R_{n}(f, g),
$$

where $\left|R_{n}(f, g)\right| \leq C\left|t_{n}\right|\left\|G_{n}^{M} f\right\|_{\Sigma}\left\|G_{n}^{N} g\right\|_{\Sigma}$.

Remark. We regard this as an "approximate adjoint" formula; note that $e^{-i t H}$ is not actually defined on all of $\dot{H}^{1}$. It follows from Lemma 4.8 that

$$
\lim _{n \rightarrow \infty}\left\langle\left(G_{n}^{N}\right)^{-1} e^{-i t_{n} H} G_{n}^{M} f, g\right\rangle_{\dot{H}^{1}}=\lim _{n \rightarrow \infty}\left\langle f,\left(G_{n}^{M}\right)^{-1} e^{i t_{n} H} G_{n}^{N} g\right\rangle_{\dot{H}^{1}}
$$

for fixed $f, g \in \Sigma$. The content of this lemma lies in the quantitative error bound.

Proof. From the identities (2.6), we obtain the commutator estimate

$$
\left\|\left[\nabla, e^{-i t H}\right]\right\|_{\Sigma \rightarrow L^{2}}=O(t)
$$

By straightforward manipulations, we obtain

$$
\left\langle\left(G_{n}^{N}\right)^{-1} e^{-i t_{n} H} G_{N}^{M} f, g\right\rangle_{\dot{H}^{1}}=\left\langle f,\left(G_{n}^{M}\right)^{-1} e^{i t_{n} H} G_{n}^{N} g\right\rangle_{\dot{H}^{1}}+R_{n}(f, g)
$$

where $R_{n}(f, g)=\left\langle\left[\nabla, e^{-i t_{n} H}\right] G_{n}^{M} f, \nabla G_{n}^{N} g\right\rangle_{L^{2}}-\left\langle\nabla G_{n}^{M} f,\left[\nabla, e^{i t_{n} H}\right] G_{n}^{N} g\right\rangle_{L^{2}}$. The claim follows from Cauchy-Schwartz and the above commutator estimate.

The next lemma is a converse to Lemma 4.8,

Lemma 4.11 (Weak convergence). Assume the frames $\mathcal{F}^{M}=\left\{\left(t_{n}^{M}, x_{n}, M_{n}\right)\right\}$ and $\mathcal{F}^{N}=\left\{\left(t_{n}^{N}, y_{n}, N_{n}\right)\right\}$ are orthogonal. Then, for any $f \in \Sigma$,

$$
\left(e^{-i t_{n}^{N} H} G_{n}^{N}\right)^{-1} e^{-i t_{n}^{M} H} G_{n}^{M} f \rightarrow 0 \text { weakly in } \dot{H}^{1} .
$$

Proof. Put $t_{n}=t_{n}^{M}-t_{n}^{N}$, and suppose that $\left|M_{n}^{2} t_{n}\right| \rightarrow \infty$. Then

$$
\left\|\left(G_{n}^{N}\right)^{-1} e^{-i t_{n} H} G_{n}^{M} f\right\|_{L^{\frac{2 d}{d-2}}} \rightarrow 0
$$

for $f \in C_{c}^{\infty}$ by a change of variables and the dispersive estimate, thus for general $f \in$ $\Sigma$ by a density argument. Therefore $\left(G_{n}^{N}\right)^{-1} e^{-i t_{n} H} G_{n}^{M} f$ converges weakly in $\dot{H}^{1}$ to 0 . We consider next the case where $M_{n}^{2} t_{n} \rightarrow t_{\infty} \in \mathbf{R}$. The orthogonality of $\mathcal{F}^{M}$ and $\mathcal{F}^{N}$ implies that either $N_{n}^{-1} M_{n}$ converges to 0 or $\infty$, or $M_{n}\left|x_{n}-y_{n}\right|$ diverges as $n \rightarrow \infty$. In either case, one verifies easily that the operators $\left(G_{n}^{N}\right)^{-1} G_{n}^{M}$ converge to zero in the weak operator topology on $B\left(\dot{H}^{1}, \dot{H}^{1}\right)$. Applying Lemma 4.8 we see that $\left(G_{n}^{N}\right)^{-1} e^{-i t_{n} H} G_{n}^{M} f=\left(G_{n}^{N}\right)^{-1} G_{n}^{M}\left(G_{n}^{M}\right)^{-1} e^{-i t_{n} H} G_{n}^{M} f$ converges to zero weakly in $\dot{H}^{1}$.

Corollary 4.12. Let $\left\{\left(t_{n}^{M}, x_{n}, M_{n}, M_{n}^{\prime}\right)\right\}$ and $\left\{\left(t_{n}^{N}, y_{n}, N_{n}, N_{n}^{\prime}\right)\right\}$ be augmented frames such that $\left\{\left(t_{n}^{M}, x_{n}, M_{n}\right)\right\}$ and $\left\{\left(t_{n}^{N}, y_{n}, N_{n}\right)\right\}$ are orthogonal. Let $G_{n}^{M}, S_{n}^{M}$ and $G_{n}^{N}, S_{n}^{N}$ be the associated operators. Then

$$
\left(e^{-i t_{n}^{N} H} G_{n}^{N}\right)^{-1} e^{-i t_{n}^{M} H} G_{n}^{M} S_{n}^{M} \phi \rightarrow 0 \quad \text { in } \quad \dot{H}^{1}
$$

whenever $\phi \in H^{1}$ if $\mathcal{F}^{M}$ is of type 2a and $\phi \in \dot{H}^{1}$ if $\mathcal{F}^{M}$ is of type 2b. 
Proof. If $\phi \in C_{c}^{\infty}$, then $S_{n}^{M} \phi=\phi$ for all large $n$, and the claim follows from Lemma 4.11. The case of general $\phi$ in $H^{1}$ or $\dot{H}^{1}$ then follows from an approximation argument similar to the one used in the proof of Corollary 4.9.

4.3. End of Proof of Inverse Strichartz. We return to the proof of Proposition 4.3 Let us pause briefly to assess our progress. We have thus far identified a frame $\left\{\left(t_{n}, x_{n}, N_{n}, N_{n}^{\prime}\right)\right\}$ and an associated profile $\phi_{n}$ such that the sequence $N_{n}^{2} t_{n}$ has a limit in $[-\infty, \infty]$ as $n \rightarrow \infty$. The $\phi_{n}$ were shown to satisfy properties (4.4) through (4.6) if either $\left(t_{n}, x_{n}, N_{n}\right)=(0,0,1)$ or $N_{n} \rightarrow \infty$ and $N_{n}^{2} t_{n} \rightarrow \pm \infty$. Thus, it remains to prove that if $N_{n} \rightarrow \infty$ and $N_{n}^{2} t_{n}$ remains bounded, then we may modify the frame so that $t_{n}$ is identically zero and find a profile $\phi_{n}$ corresponding to this new frame which satisfies all the properties asserted in the proposition. The following lemma will therefore complete the proof of Proposition 4.3

Lemma 4.13. Let $f_{n} \in \Sigma$ satisfy the hypotheses of Proposition 4.3. Suppose $\left\{\left(t_{n}, x_{n}, N_{n}, N_{n}^{\prime}\right)\right\}$ is an augmented frame with $N_{n} \rightarrow \infty$ and $N_{n}^{2} t_{n} \rightarrow t_{\infty} \in$ $\mathbf{R}$ as $n \rightarrow \infty$. Then there is a profile $\phi_{n}^{\prime}=G_{n} S_{n} \phi^{\prime}$ associated to the frame $\left\{\left(0, x_{n}, N_{n}, N_{n}^{\prime}\right)\right\}$ such that properties (4.4), (4.5), and (4.6) hold with $\phi_{n}^{\prime}$ in place of $\phi_{n}$.

Proof. Let $\phi_{n}=e^{i t_{n} H} G_{n} S_{n} \phi$ be the profile defined by (4.10). We have already seen that $\phi_{n}$ satisfies properties (4.4) and (4.6), and that

$$
\phi=\dot{\mathrm{H}}^{1}-\mathrm{w}-\lim _{n \rightarrow \infty} G_{n}^{-1} e^{-i t_{n} H} f_{n} .
$$

As the sequence $G_{n}^{-1} f_{n}$ is bounded in $\dot{H}^{1}$, it has a weak subsequential limit

$$
\phi^{\prime}=\dot{\mathrm{H}}^{1}-\mathrm{w}-\lim _{n \rightarrow \infty} G_{n}^{-1} f_{n} .
$$

For any $\psi \in C_{c}^{\infty}$, we apply Lemma 4.10 with $f=G_{n}^{-1} e^{-i t_{n} H} f_{n}$ to see that

$$
\begin{aligned}
\left\langle\phi^{\prime}, \psi\right\rangle_{\dot{H}^{1}} & =\lim _{n \rightarrow \infty}\left\langle G_{n}^{-1} f_{n}, \psi\right\rangle_{\dot{H}^{1}}=\lim _{n \rightarrow \infty}\left\langle G_{n}^{-1} e^{i t_{n} H} G_{n} G_{n}^{-1} e^{-i t_{n} H} f_{n}, \psi\right\rangle_{\dot{H}^{1}} \\
& =\lim _{n \rightarrow \infty}\left\langle G_{n}^{-1} e^{-i t_{n} H} f_{n}, G_{n}^{-1} e^{-i t_{n} H} G_{n} \psi\right\rangle_{\dot{H}^{1}}=\left\langle\phi, U_{\infty} \psi\right\rangle_{\dot{H}^{1}},
\end{aligned}
$$


Lemma 4.8. As $U_{\infty}$ is unitary on $\dot{H}^{1}$, we have the relation $\phi=U_{\infty} \phi^{\prime}$.

Put $\phi_{n}^{\prime}=G_{n} S_{n} \phi^{\prime}$. By Corollary 4.9

$$
\left\|\phi_{n}-\phi_{n}^{\prime}\right\|_{\Sigma}=\left\|e^{i t_{n} H} G_{n} S_{n} \phi-G_{n} S_{n} U_{\infty}^{-1} \phi\right\|_{\Sigma} \rightarrow 0 \quad \text { as } \quad n \rightarrow \infty .
$$

Hence $\phi_{n}^{\prime}$ inherits property (4.4) from $\phi_{n}$. The same proof as for $\phi_{n}$ shows that $\Sigma$ decoupling (4.6) holds as well. It remains to verify the last decoupling property (4.5). As $G_{n}^{-1} f_{n}$ converges weakly in $\dot{H}^{1}$ to $\phi^{\prime}$, by Rellich-Kondrashov and a diagonalization argument we may assume after passing to a subsequence that $G_{n}^{-1} f_{n}$ converges to $\phi^{\prime}$ almost everywhere on $\mathbf{R}^{d}$. By the Lemma 2.6, the observation that $\lim _{n \rightarrow \infty}\left\|G_{n} S_{n} \phi^{\prime}-G_{n} \phi^{\prime}\right\|_{\frac{2 d}{d-2}}=0$, and a change of variables, we have

$$
\begin{aligned}
& \lim _{n \rightarrow \infty}\left[\left\|f_{n}\right\|_{\frac{2 d}{d-2}}^{\frac{2 d}{d-2}}-\left\|f_{n}-\phi_{n}^{\prime}\right\|_{\frac{2 d}{d-2}}^{\frac{2 d}{d-2}}-\left\|\phi_{n}^{\prime}\right\|_{\frac{2 d}{d-2}}^{\frac{2 d}{d-2}}\right] \\
& =\lim _{n \rightarrow \infty}\left[\left\|G_{n}^{-1} f_{n}\right\|_{\frac{2 d}{d-2}}^{\frac{2 d}{d-2}}-\left\|G_{n}^{-1} f_{n}-\phi^{\prime}\right\|_{\frac{2 d}{d-2}}^{\frac{2 d}{d-2}}-\left\|\phi^{\prime}\right\|_{\frac{2 d}{d-2}}^{\frac{2 d d}{d-2}}\right] \\
& =0 .
\end{aligned}
$$


Remark. As $\lim _{n \rightarrow \infty}\left\|\phi_{n}-\phi_{n}^{\prime}\right\|_{\Sigma}=0$, we see by Sobolev embedding that the decoupling (4.5) also holds for the original profile $\phi_{n}=e^{i t_{n} H} G_{n} S_{n} \phi$ with nonzero time parameter $t_{n}$.

4.4. Linear profile decomposition. We are ready to write down the linear profile decomposition. As before, $I$ will denote a fixed interval containing 0 of length at most 1, and all spacetime norms are taken over $I \times \mathbf{R}^{d}$ unless indicated otherwise.

Proposition 4.14. Let $f_{n}$ be a bounded sequence in $\Sigma$. After passing to a subsequence, there exists $J^{*} \in\{0,1, \ldots\} \cup\{\infty\}$ such that for each finite $1 \leq j \leq J^{*}$, there exist an augmented frame $\mathcal{F}^{j}=\left\{\left(t_{n}^{j}, x_{n}^{j}, N_{n}^{j},\left(N_{n}^{j}\right)^{\prime}\right)\right\}$ and a function $\phi^{j}$ with the following properties.

- Either $t_{n}^{j} \equiv 0$ or $\left(N_{n}^{j}\right)^{2}\left(t_{n}^{j}\right) \rightarrow \pm \infty$ as $n \rightarrow \infty$.

- $\phi^{j}$ belongs to $\Sigma, H^{1}$, or $\dot{H}^{1}$ depending on whether $\mathcal{F}^{j}$ is of type [1, 2a, or 26, respectively.

For each finite $J \leq J^{*}$, we have a decomposition

$$
f_{n}=\sum_{j=1}^{J} e^{i t_{n}^{j} H} G_{n}^{j} S_{n}^{j} \phi^{j}+r_{n}^{J},
$$

where $G_{n}^{j}, S_{n}^{j}$ are the $\dot{H}^{1}$-isometry and spatial cutoff operators associated to $\mathcal{F}^{j}$. Writing $\phi_{n}^{j}$ for $e^{i t_{n}^{j} H} G_{n}^{j} S_{n}^{j} \phi^{j}$, this decomposition has the following properties:

$$
\begin{gathered}
\left(G_{n}^{J}\right)^{-1} e^{-i t_{n}^{J} H} r_{n}^{J} \stackrel{\dot{H}^{1}}{\rightarrow} 0 \quad \text { for all } J \leq J^{*}, \\
\sup _{J} \lim _{n \rightarrow \infty}\left|\left\|f_{n}\right\|_{\Sigma}^{2}-\sum_{j=1}^{J}\left\|\phi_{n}^{j}\right\|_{\Sigma}^{2}-\left\|r_{n}^{J}\right\|_{\Sigma}^{2}\right|=0, \\
\sup _{J} \lim _{n \rightarrow \infty}\left|\left\|f_{n}\right\|_{L_{x}^{\frac{2 d}{d-2}}}^{\frac{2 d}{d-2}}-\sum_{j=1}^{J}\left\|\phi_{n}^{j}\right\|_{L_{x}^{\frac{2 d}{d-2}}}^{\frac{2 d}{d-2}}-\left\|r_{n}^{J}\right\|_{L_{x}^{\frac{2 d}{d-2}}}^{\frac{2 d}{d-2}}\right|=0 .
\end{gathered}
$$

Whenever $j \neq k$, the frames $\left\{\left(t_{n}^{j}, x_{n}^{j}, N_{n}^{j}\right)\right\}$ and $\left\{\left(t_{n}^{k}, x_{n}^{k}, N_{n}^{k}\right)\right\}$ are orthogonal:

$$
\lim _{n \rightarrow \infty} \frac{N_{n}^{j}}{N_{n}^{k}}+\frac{N_{n}^{k}}{N_{n}^{j}}+N_{n}^{j} N_{n}^{k}\left|t_{n}^{j}-t_{n}^{k}\right|+\sqrt{N_{n}^{j} N_{n}^{k}}\left|x_{n}^{j}-x_{n}^{k}\right|=\infty .
$$

Finally, we have

$$
\lim _{J \rightarrow J^{*}} \limsup _{n \rightarrow \infty}\left\|e^{-i t_{n} H} r_{n}^{J}\right\|_{L_{t, x}^{\frac{2(d+2)}{d-2}}}=0,
$$

Remark. One can also show a posteriori using (4.17) and (4.18) the fact, which we will neither prove nor use, that

$$
\sup _{J} \lim _{n \rightarrow \infty}\left|\left\|e^{-i t H} f_{n}\right\|_{L_{t, x}}^{\frac{2(d+2)}{d-2}}-\sum_{j=1}^{J}\left\|e^{-i t H} \phi_{n}^{j}\right\|_{L_{t, x}}^{\frac{2(d+2)}{d-2}}-\left\|e^{\frac{2(d+2)}{d-2}}-w_{n}^{J}\right\|_{L_{t, x}}^{\frac{2(d-2)}{d-2}}\right|=0 .
$$

The argument uses similar ideas as in the proofs of [17][Lemma 2.7] or Lemma 6.3] we omit the details.

Proof. We proceed inductively using Proposition 4.3 Let $r_{n}^{0}=f_{n}$. Assume that we have a decomposition up to level $J \geq 0$ obeying properties (4.14) through (4.16). After passing to a subsequence, we may define

$$
A_{J}=\lim _{n}\left\|r_{n}^{J}\right\|_{\Sigma} \quad \text { and } \quad \varepsilon_{J}=\lim _{n}\left\|e^{-i t_{n} H} r_{n}^{J}\right\|_{L_{t, x} \frac{2(d+2)}{d-2}} .
$$


If $\varepsilon_{J}=0$, stop and set $J^{*}=J$. Otherwise we apply Proposition 4.3 to the sequence $r_{n}^{J}$ to obtain a frame $\left(t_{n}^{J+1}, x_{n}^{J+1}, N_{n}^{J+1},\left(N_{n}^{J+1}\right)^{\prime}\right)$ and functions

$$
\phi^{J+1} \in \dot{H}^{1}, \quad \phi_{n}^{J+1}=e^{i t_{n}^{J+1} H} G_{n}^{J+1} S_{n}^{J+1} \phi^{J+1} \in \Sigma
$$

which satisfy the conclusions of Proposition 4.3. In particular $\phi^{J+1}$ is the $\dot{H}^{1}$ weak limit of $\left(G_{n}^{J+1}\right)^{-1} e^{-i t_{n}^{J+1} H} r_{n}^{J}$. Let $r_{n}^{J+1}=r_{n}^{J}-\phi_{n}^{J+1}$. By the induction hypothesis, (4.15) and (4.16) are satisfied with $J$ replaced by $J+1$. We also have

$$
\left(G_{n}^{J+1}\right)^{-1} e^{-i t_{n}^{J+1} H} r_{n}^{J+1}=\left[\left(G_{n}^{J+1}\right)^{-1} e^{-i t_{n}^{J+1} H} r_{n}^{J}-\phi^{J+1}\right]+\left(1-S_{n}^{J+1}\right) \phi^{J+1} .
$$

As $n \rightarrow \infty$, the first term goes to zero weakly in $\dot{H}^{1}$ while the second term goes to zero strongly. Thus (4.14) holds at level $J+1$ as well. After passing to a subsequence, we may define

$$
A_{J+1}=\lim _{n}\left\|r_{n}^{J+1}\right\|_{\Sigma} \quad \text { and } \quad \varepsilon_{J+1}=\lim _{n}\left\|e^{-i t H} r_{n}^{J+1}\right\|_{L_{t, x}^{\frac{2(d+2)}{d-2}}} .
$$

If $\varepsilon_{J+1}=0$, stop and set $J^{*}=J+1$. Otherwise continue the induction. If the algorithm never terminates, set $J^{*}=\infty$. From (4.15) and (4.16), the parameters $A_{J}$ and $\varepsilon_{J}$ satisfy the inequality

$$
A_{J+1}^{2} \leq A_{J}^{2}\left[1-C\left(\frac{\varepsilon_{J}}{A_{J}}\right)^{\frac{d(d+2)}{4}}\right] .
$$

If $\limsup _{J \rightarrow J^{*}} \varepsilon_{J}=\varepsilon_{\infty}>0$, then as $A_{J}$ are decreasing there would exist infinitely many $J$ 's so that

$$
A_{J+1}^{2} \leq A_{J}^{2}\left[1-C\left(\frac{\varepsilon_{\infty}}{A_{0}}\right)^{\frac{d(d+2)}{4}}\right]
$$

which implies that $\lim _{J \rightarrow J^{*}} A_{J}=0$. But this contradicts the Strichartz inequality which dictates that $\limsup _{J \rightarrow J^{*}} A_{J} \gtrsim \lim \sup _{J \rightarrow J^{*}} \varepsilon_{J}=\varepsilon_{0}$. We conclude that

$$
\lim _{J \rightarrow J^{*}} \varepsilon_{J}=0 \text {. }
$$

Thus (4.18) holds.

It remains to prove the assertion (4.17). Suppose otherwise, and let $j<k$ be the first two indices for which $\mathcal{F}^{j}$ and $\mathcal{F}^{k}$ are equivalent. Thus $\mathcal{F}^{\ell}$ and $\mathcal{F}^{k}$ are orthogonal for all $j<\ell<k$. By the construction of the profiles, we have

$$
r_{n}^{j-1}=e^{i t_{n}^{j} H} G_{n}^{j} S_{n}^{j} \phi^{j}+e^{i t_{n}^{k} H} G_{n}^{k} S_{n}^{k} \phi^{k}+\sum_{j<\ell<k} e^{i t_{n}^{\ell} H} G_{n}^{\ell} S_{n}^{\ell} \phi^{\ell}+r_{n}^{k},
$$

thus

$$
\begin{aligned}
& \left(e^{i t_{n}^{j} H} G_{n}^{j}\right)^{-1} r_{n}^{j-1}=\left(e^{i t_{n}^{j} H} G_{n}^{j}\right)^{-1} e^{i t_{n}^{j} H} G_{n}^{j} S_{n}^{j} \phi^{j}+\left(e^{i t_{n}^{j} H} G_{n}^{j}\right)^{-1} e^{i t_{n}^{k} H} G_{n}^{k} S_{n}^{k} \phi^{k} \\
& +\sum_{j<\ell<k}\left(e^{i t_{n}^{j} H} G_{n}^{j}\right)^{-1} e^{i t_{n}^{\ell} H} G_{n}^{\ell} S_{n}^{\ell} \phi^{\ell}+\left(e^{i t_{n}^{j} H} G_{n}^{j}\right)^{-1} r_{n}^{k} .
\end{aligned}
$$

As $n \rightarrow \infty$, the left side converges to $\phi^{j}$ weakly in $\dot{H}^{1}$. On the right side, we apply Corollary 4.9 to see that the first and second terms converge in $\dot{H}^{1}$ to $\phi^{j}$ and $U_{\infty}^{j k} \phi^{k}$, respectively, for some isomorphism $U_{\infty}^{j k}$ of $\dot{H}^{1}$. By Corollary 4.12, each of the terms in the summation converges to zero weakly in $\dot{H}^{1}$. Taking for granted the claim that

$$
\left(e^{i t_{n}^{j} H} G_{n}^{j}\right)^{-1} r_{n}^{k} \rightarrow 0 \quad \text { weakly in } \dot{H}^{1}
$$

it follows that

$$
\phi^{j}=\phi^{j}+U_{\infty}^{j k} \phi^{k}
$$


so $\phi^{k}=0$, which contradicts the nontriviality of $\phi^{k}$. Therefore, the proof of the proposition will be complete once we verify the weak limit (4.19). As that sequence is bounded in $\dot{H}^{1}$, it suffices to check that

$$
\left\langle\left(e^{i t_{n}^{j} H} G_{n}^{j}\right)^{-1} r_{n}^{k}, \psi\right\rangle_{\dot{H}^{1}} \rightarrow 0 \text { for any } \psi \in C_{c}^{\infty}\left(\mathbf{R}^{d}\right) .
$$

Writing $\left(e^{i t_{n}^{j} H} G_{n}^{j}\right)^{-1} r_{n}^{k}=\left(e^{i t_{n}^{j} H} G_{n}^{j}\right)^{-1}\left(e^{i t_{n}^{k} H} G_{n}^{k}\right)\left(e^{i t_{n}^{k} H} G_{n}^{k}\right)^{-1} r_{n}^{k}$, we apply Lemma 4.10 and the weak limit (4.14) to see that

$$
\begin{aligned}
\lim _{n \rightarrow \infty}\left\langle\left(e^{i t_{n}^{j} H} G_{n}^{j}\right)^{-1} r_{n}^{k}, \psi\right\rangle_{\dot{H}^{1}} & =\lim _{n \rightarrow \infty}\left\langle\left(e^{i t_{n}^{k} H} G_{n}^{k}\right)^{-1} r_{n}^{k},\left(e^{i t_{n}^{k} H} G_{n}^{k}\right)^{-1}\left(e^{i t_{n}^{j} H} G_{n}^{j}\right) \psi\right\rangle_{\dot{H}^{1}} \\
& =\lim _{n \rightarrow \infty}\left\langle\left(G_{n}^{k}\right)^{-1} e^{-i t_{n}^{k} H} r_{n}^{k},\left(U_{\infty}^{j k}\right)^{-1} \psi\right\rangle_{\dot{H}^{1}} \\
& =0 .
\end{aligned}
$$

\section{The CASE OF CONCENTRATED INITIAL DATA}

The next step in the proof of Theorem 1.2 is to establish wellposedness when the initial data consists of a highly concentrated "bubble". The picture to keep in mind is that of a single profile $\phi_{n}^{j}$ in Proposition 4.14 as $n \rightarrow \infty$. In the next section we combine this special case with the profile decomposition to treat general initial data. Although we state the following result as a conditional one to permit a unified exposition, by Theorem 1.1 the result is unconditionally true in most cases.

Proposition 5.1. Let $I=[-1,1]$. Assume that Conjecture 1.1 holds. Suppose

$$
\mathcal{F}=\left\{\left(t_{n}, x_{n}, N_{n}, N_{n}^{\prime}\right)\right\}
$$

is an augmented frame with $t \in I$ and $N_{n} \rightarrow \infty$, such that either $t_{n} \equiv 0$ or $N_{n}^{2} t_{n} \rightarrow \pm \infty$; that is, $\mathcal{F}$ is type $2 a$ or $2 b$ in Definition 4.2. Let $G_{n}, \tilde{G}_{n}$, and $S_{n}$ be the associated operators as defined in (4.1) and (4.2). Suppose $\phi$ belongs to $H^{1}$ or $\dot{H}^{1}$ depending on whether $\mathcal{F}$ is type $2 a$ or $2 b$ respectively. Then, for $n$ sufficiently large, there is a unique solution $u_{n}: I \times \mathbf{R}^{d} \rightarrow \mathbf{C}$ to the defocusing equation (1.1), $\mu=1$, with initial data

$$
u_{n}(0)=e^{i t_{n} H} G_{n} S_{n} \phi
$$

This solution satisfies a spacetime bound

$$
\limsup _{n \rightarrow \infty} S_{I}\left(u_{n}\right) \leq C\left(E\left(u_{n}\right)\right) .
$$

Suppose in addition that $\left\{\left(q_{k}, r_{k}\right)\right\}$ is any finite collection of admissible pairs with $2<r_{k}<d$. Then for each $\varepsilon>0$ there exists $\psi^{\varepsilon} \in C_{c}^{\infty}\left(\mathbf{R} \times \mathbf{R}^{d}\right)$ such that

$$
\limsup _{n \rightarrow \infty} \sum_{k}\left\|u_{n}-\tilde{G}_{n}\left[e^{-\frac{i t N_{n}^{-2}\left|x_{n}\right|^{2}}{2}} \psi^{\varepsilon}\right]\right\|_{L_{t}^{q_{k}} \Sigma_{x}^{r_{k}}\left(I \times \mathbf{R}^{d}\right)}<\varepsilon .
$$

Assuming also that $\|\nabla \phi\|_{L^{2}}<\|\nabla W\|_{L^{2}}$ and $E_{\Delta}(\phi)<E_{\Delta}(W)$, we have the same conclusion as above for the focusing equation (1.1), $\mu=-1$.

The proof proceeds in several steps. First we construct an approximate solution on $I$ in the sense of Proposition 3.3. Roughly speaking, when $N_{n}$ is large and $t=O\left(N_{n}^{-2}\right)$, solutions to (1.1) are well-approximated up to a phase factor by solutions to the energy-critical NLS with no potential, which by Conjecture 1.1 exist globally and scatter. In the long-time regime $|t|>>N_{n}^{-2}$, the solution to (1.1) has dispersed and resembles a linear evolution $e^{-i t H} \phi$ (note that we are not 
claiming scattering since we consider only a fixed finite time window). By patching these approximations together, we obtain an approximate solution over the entire time interval $I$ with arbitrarily small error as $N_{n}$ becomes large. We then invoke Proposition 3.3 to conclude that for $n$ large enough (1.1) admits a solution on $I$ with controlled spacetime bound. The last claim about approximating the solution by functions in $C_{c}^{\infty}\left(\mathbf{R} \times \mathbf{R}^{d}\right)$ will follow essentially from our construction of the approximate solutions.

We first record a basic commutator estimate. Throughout the rest of this section, $P_{\leq N}, P_{N}$ will denote the standard Littlewood-Paley projectors based on $-\Delta$.

Lemma 5.2 (Commutator estimate). Let $v$ be a global solution to

$$
\left(i \partial_{t}+\frac{1}{2} \Delta\right) v=F(v), v(0) \in \dot{H}^{1}\left(\mathbf{R}^{d}\right)
$$

where $F(z)= \pm|z|^{\frac{4}{d-2}} z$. Then on any compact time interval $I$,

$$
\lim _{N \rightarrow \infty}\left\|P_{\leq N} F(v)-F\left(P_{\leq N} v\right)\right\|_{L_{t}^{2} H_{x}^{1, \frac{2 d}{d+2}}\left(I \times \mathbf{R}^{d}\right)}=0
$$

Proof. We recall [29, Lemma 3.11] that as a consequence of the spacetime bound (1.7), $\nabla v$ is finite in all Strichartz norms:

$$
\|\nabla v\|_{S(\mathbf{R})}<C\left(\|v(0)\|_{\dot{H}^{1}}\right)<\infty .
$$

Clearly it will suffice to show separately that

$$
\begin{gathered}
\lim _{n \rightarrow \infty}\left\|P_{\leq N} F(v)-F\left(P_{\leq N} v\right)\right\|_{L_{t}^{2} L_{x}^{\frac{2 d}{d+2}}}=0, \\
\lim _{n \rightarrow \infty}\left\|\nabla\left[P_{\leq N} F(v)-F\left(P_{\leq N} v\right)\right]\right\|_{L_{t}^{2} L_{x}^{\frac{2 d}{d+2}}}=0 .
\end{gathered}
$$

Write

$$
\begin{aligned}
\left\|\nabla\left[P_{\leq N} F(v)-F\left(P_{\leq N} v\right)\right]\right\|_{L_{t}^{2} L_{x}^{\frac{2 d}{d+2}}} & \leq\left\|\nabla P_{>N} F(v)\right\|_{L_{t}^{2} L_{x}^{\frac{2 d}{d+2}}} \\
& +\left\|\nabla\left[F(v)-F\left(P_{\leq N} v\right)\right]\right\|_{L_{t}^{2} L_{x}^{\frac{2 d}{d+2}}}
\end{aligned}
$$

As $P_{>N}=1-P_{\leq N}$ and

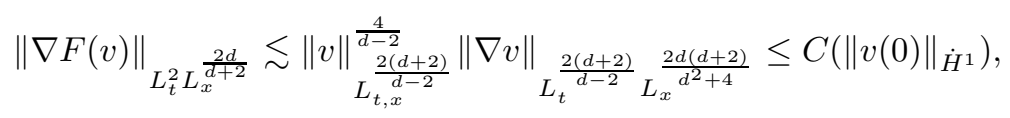

dominated convergence implies that

$$
\lim _{N \rightarrow \infty}\left\|\nabla P_{>N} F(v)\right\|_{L_{t}^{2} L_{x}^{\frac{2 d}{d+2}}}=0 .
$$

To treat the second term on the right side of (5.5), observe first that with $F(z)=$ $|z|^{\frac{4}{d-2}} z$

$$
\left|F_{z}(z)-F_{z}(w)\right|+\left|F_{\bar{z}}(z)-F_{\bar{z}}(w)\right| \lesssim\left\{\begin{array}{cc}
|z-w|\left(|z|^{\frac{6-d}{d-2}}+|w|^{\frac{6-d}{d-2}}\right), & 3 \leq d \leq 5 \\
|z-w|^{\frac{4}{d-2}}, & d \geq 6 .
\end{array}\right.
$$

Combining this with the pointwise bound

$$
\begin{aligned}
\left|\nabla\left[F(v)-F\left(P_{\leq N} v\right)\right]\right| & \leq\left(\left|F_{z}(v)-F_{z}\left(P_{\leq N} v\right)\right|+\left|F_{\bar{z}}(v)-F_{\bar{z}}\left(P_{\leq N} v\right)\right|\right)|\nabla v| \\
& +\left(\left|F_{z}\left(P_{\leq N} v\right)\right|+\left|F_{\bar{z}}\left(P_{\leq N} v\right)\right|\right)\left|\nabla P_{>N} v\right|,
\end{aligned}
$$


Hölder, and dominated convergence, when $d \geq 6$ we have

$$
\begin{aligned}
& \left\|\nabla\left[F(v)-F\left(P_{\leq N} v\right)\right]\right\|_{L_{t}^{2} L_{x}^{\frac{2 d}{d+2}}} \\
& \lesssim\left\|\left|P_{>N} v\right|^{\frac{4}{d-2}}|\nabla v|\right\|_{L_{t}^{2} L_{x}^{\frac{2 d}{d+2}}}+\left\|\left|P_{\leq N} v\right|^{\frac{4}{d-2}}\left|\nabla P_{>N} v\right|\right\|_{L_{t}^{2} L_{x}^{\frac{2 d}{d+2}}}
\end{aligned}
$$

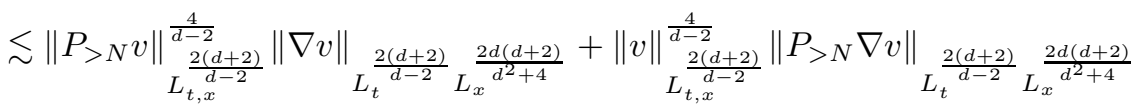

$$
\begin{aligned}
& \rightarrow 0 \text { as } N \rightarrow \infty \text {. }
\end{aligned}
$$

If $3 \leq d \leq 5$, the first term in the second line of (5.6) is replaced by

$$
\begin{aligned}
& \left\|\left|P_{>N} v\right|\left(|v|^{\frac{6-d}{d-2}}+\left|P_{\leq N} v\right|^{\frac{6-d}{d-2}}\right)|\nabla v|\right\|_{L_{t}^{2} L_{x}^{\frac{2 d}{d+2}}} \\
& \leq\left\|P_{>N} v\right\|_{L_{t, x}}^{\frac{2(d+2)}{d-2}}\|v\|_{L_{t, x}^{\frac{2(d+2}{d-2}}}^{\frac{6-d}{d-2}}\|\nabla v\|_{L_{t}^{\frac{2(d+2)}{d-2}}} \frac{2 d(d+2)}{L_{x} \frac{d^{2}+4}{d^{2}+4}}
\end{aligned}
$$

which goes to 0 by dominated convergence. This establishes (5.4). The proof of (5.3) is similar. Write

$$
\left\|P_{\leq N} F(v)-F\left(P_{\leq N} v\right)\right\|_{L_{t}^{2} L_{x}^{\frac{2 d}{d+2}}} \leq\left\|P_{>N} F(v)\right\|_{L_{t}^{2} L_{x}^{\frac{2 d}{d+2}}}+\left\|F(v)-F\left(P_{\leq N} v\right)\right\|_{L_{t}^{2} L_{x}^{\frac{2 d}{d+2}}} .
$$

By Hölder, Bernstein, and the chain rule,

$$
\left\|P_{>N} F(v)\right\|_{L_{t}^{2} L_{x}^{\frac{2 d}{d+2}}} \lesssim N^{-1}\left\|\left.v\right|_{L_{t, x}^{\frac{2(d+2)}{d-2}}} ^{\frac{4}{d-2}}\right\| \nabla v \|_{L_{t}^{\frac{2(d+2)}{d-2}}} \frac{\frac{2 d(d+2)}{L_{x} d^{2}+4}}{\left(N^{2}\right.}=O\left(N^{-1}\right) .
$$

Using Bernstein, Hölder, and Sobolev embedding, and the pointwise bound

$$
\left|F(v)-F\left(P_{\leq N} v\right)\right| \lesssim\left|P_{>N} v\right|\left(|v|^{\frac{4}{d-2}}+\left|P_{\leq N} v\right|^{\frac{4}{d-2}}\right),
$$

we obtain

$$
\begin{aligned}
\left\|F(v)-F\left(P_{\leq N} v\right)\right\|_{L_{t}^{2} L_{x}^{\frac{2 d}{d+2}}} & \leq\left\|\left(|v|^{\frac{4}{d-2}}+\left|P_{\leq N} v\right|^{\frac{4}{d^{-2}}}\right) P_{>N} v\right\|_{L_{t}^{2} L_{x}^{\frac{2 d}{d+2}}} \\
& \lesssim|I|\left(\|\nabla v\|_{L_{t}^{\infty} L_{x}^{2}}^{\frac{4}{d-2}}++\|\nabla v\|_{L_{t}^{\infty-2} L_{x}^{2}}^{\frac{d-2}{d-2}}\right)\left\|\nabla P_{>N} v\right\|_{L_{t}^{\infty} L_{x}^{2}} .
\end{aligned}
$$

As $v \in C_{t}^{0} \dot{H}_{x}^{1}\left(I \times \mathbf{R}^{d}\right)$, the orbit $\{v(t)\}_{t \in I}$ is compact in $\dot{H}^{1}\left(\mathbf{R}^{d}\right)$. The Riesz characterization of $L^{2}$ compactness therefore implies that the right side goes to 0 as $N \rightarrow \infty$.

Now suppose that $\phi_{n}=e^{i t_{n} H} G_{n} S_{n} \phi$ as in the statement of Proposition [5.1. If $\mu=-1$, assume also that $\|\phi\|_{\dot{H}^{1}}<\|W\|_{\dot{H}^{1}}, E(\phi)<E_{\Delta}(W)$. We first construct "quasi-approximate" solutions $\tilde{v}_{n}$ which obey all of the conditions of the Proposition 3.3 except possibly the hypothesis in (3.2) about matching initial data. A slight modification of the $\tilde{v}_{n}$ will then yield genuine approximate solutions.

If $t_{n} \equiv 0$, let $v$ be the solution to the potential-free problem (1.6) provided by Conjecture 1.1 with $v(0)=\phi$. If $N_{n}^{2} t_{n} \rightarrow \pm \infty$, let $v$ be the solution to (1.6) which scatters in $\dot{H}^{1}$ to $e^{\frac{i t \Delta}{2}} \phi$ as $t \rightarrow \mp \infty$. Note the reversal of signs.

Put

$$
\tilde{N}_{n}^{\prime}=\left(\frac{N_{n}}{N_{n}^{\prime}}\right)^{\frac{1}{2}}
$$


Let $T>0$ denote a large constant to be chosen later, and define

$$
\tilde{v}_{n}^{T}(t)=\left\{\begin{array}{cc}
e^{-\frac{i t\left|x_{n}\right|^{2}}{2}} \tilde{G}_{n}\left[S_{n} P_{\leq \tilde{N}_{n}^{\prime}} v\right]\left(t+t_{n}\right) & |t| \leq T N_{n}^{-2} \\
e^{-i\left(t-T N_{n}^{-2}\right) H} \tilde{v}_{n}^{T}\left(T N_{n}^{-2}\right), & T N_{n}^{-2} \leq t \leq 2 \\
e^{-i\left(t+T N_{n}^{-2}\right) H} \tilde{v}_{n}^{T}\left(-T N_{n}^{-2}\right), & -2 \leq t \leq-T N_{n}^{-2}
\end{array}\right.
$$

The time translation by $t_{n}$ is needed to undo the time translation built into the operator $\tilde{G}_{n}$; see (4.1). We will suppress the superscript $T$ unless we need to emphasize the role of that parameter. Introducing the notation

$$
\begin{aligned}
v_{n}(t, x) & =\left[\tilde{G}_{n} v\right]\left(t+t_{n}, x\right)=N_{n}^{\frac{d-2}{2}} v\left(N_{n}^{2} t, N_{n}\left(x-x_{n}\right)\right), \\
\chi_{n}(x) & =\chi\left(N_{n}^{\prime}\left(x-x_{n}\right)\right),
\end{aligned}
$$

where $\chi$ is the function used to define the spatial cutoff operator $S_{n}$ in (4.2), and using the identity $\tilde{G}_{n} \chi=\chi_{n} \tilde{G}_{n}$, we can also write the top expression in (5.8) as

$$
\tilde{v}_{n}(t)=e^{-\frac{i t\left|x_{n}\right|^{2}}{2}} \chi_{n} P_{\leq \tilde{N}_{n}^{\prime} N_{n}} v_{n}, \quad|t| \leq T N_{n}^{-2} .
$$

As discussed previously, inside the "potential-free" window $\tilde{v}_{n}$ is essentially a modulated solution to (1.6) with cutoffs applied in both space, to place the solution in $C_{t} \Sigma_{x}$, and frequency, to enable taking an extra derivative in the error analysis below.

On the time interval $|t| \leq T N_{n}^{-2}$, we use Lemma 4.2 and the fact that $\|v\|_{L_{t}^{\infty} \dot{H}_{x}^{1}} \lesssim$ $\|\phi\|_{\dot{H}^{1}}$ to deduce

$$
\limsup _{n}\left\|\tilde{v}_{n}\right\|_{L_{t}^{\infty} \Sigma_{x}\left(|t| \leq T N_{n}^{-2}\right)} \lesssim\|\phi\|_{\dot{H}^{1}}
$$

therefore

$$
\limsup _{n}\left\|\tilde{v}_{n}\right\|_{L_{t}^{\infty} \Sigma_{x}([-2,2])} \lesssim\|\phi\|_{\dot{H}^{1}} .
$$

From (1.7), (5.9), and Strichartz, we obtain

$$
\left\|\tilde{v}_{n}\right\|_{L_{t, x}^{\frac{2(d+2)}{d-2}}\left([-2,2] \times \mathbf{R}^{d}\right)} \leq C\left(\|\phi\|_{\dot{H}^{1}}\right) \quad \text { for } n \text { large. }
$$

Let

We show that

$$
e_{n}=\left(i \partial_{t}-H\right) \tilde{v}_{n}-F\left(\tilde{v}_{n}\right)
$$

$$
\lim _{T \rightarrow \infty} \limsup _{n \rightarrow \infty}\left\|H^{\frac{1}{2}} e_{n}\right\|_{N([-2,2])}=0,
$$

so that by taking $T$ large enough the $\tilde{v}_{n}$ will satisfy the second error condition in (3.2) for all $n$ sufficiently large. Our first task is to deal with the time interval $|t| \leq T N_{n}^{-2}$.

Lemma 5.3. $\lim _{T \rightarrow \infty} \lim \sup _{n \rightarrow \infty}\left\|H^{\frac{1}{2}} e_{n}\right\|_{N\left(|t| \leq T N_{n}^{-2}\right)}=0$.

Proof. When $-T N_{n}^{-2} \leq t \leq T N_{n}^{-2}$, we compute

$$
\begin{aligned}
e_{n} & =e^{-\frac{i t\left|x_{n}\right|^{2}}{2}}\left[\chi_{n} P_{\leq \tilde{N}_{n}^{\prime} N_{n}} F\left(v_{n}\right)-\chi_{n}^{\frac{d+2}{d-2}} F\left(P_{\leq \tilde{N}_{n}^{\prime} N_{n}} v_{n}\right)\right. \\
& \left.+\frac{\left|x_{n}\right|^{2}-|x|^{2}}{2}\left(P_{\leq \tilde{N}_{n}^{\prime} N_{n}} v_{n}\right) \chi_{n}+\frac{1}{2}\left(P_{\leq \tilde{N}_{n}^{\prime} N_{n}} v_{n}\right) \Delta \chi_{n}+\left(\nabla P_{\leq \tilde{N}_{n}^{\prime} N_{n}} v_{n}\right) \cdot \nabla \chi_{n}\right] \\
& =e^{-\frac{i t\left|x_{n}\right|^{2}}{2}}[(a)+(b)+(c)+(d)],
\end{aligned}
$$


CASEY JAO

and estimate each term separately in the dual Strichartz space $N\left(\left\{|t| \leq T N_{n}^{-2}\right\}\right)$. Write

$$
\begin{aligned}
(a) & =\chi_{n} P_{\leq \tilde{N}_{n}^{\prime} N_{n}} F\left(v_{n}\right)-\chi_{n}^{\frac{d+2}{d-2}} F\left(P_{\leq \tilde{N}_{n}^{\prime} N_{n}} v_{n}\right) \\
& =\chi_{n}\left[P_{\leq \tilde{N}_{n}^{\prime} N_{n}} F\left(v_{n}\right)-F\left(P_{\tilde{N}_{n}^{\prime} N_{n}} v_{n}\right)\right]+\chi_{n}\left(1-\chi_{n}^{\frac{4}{d-2}}\right) F\left(P_{\leq \tilde{N}_{n}^{\prime} N_{n}} v_{n}\right) \\
& =\left(a^{\prime}\right)+\left(a^{\prime \prime}\right) .
\end{aligned}
$$

By the Leibniz rule and a change of variables,

$$
\begin{aligned}
& \left\|\nabla\left(a^{\prime}\right)\right\|_{L_{t}^{2} L_{x}^{\frac{2 d}{d+2}}\left(|t| \leq T N_{n}^{-2}\right)} \\
& \leq\left\|\nabla\left[P_{\leq \tilde{N}_{n}^{\prime}} F(v)-F\left(P_{\leq \tilde{N}_{n}^{\prime}} v\right)\right]\right\|{ }_{L_{t}^{2} L_{x}^{\frac{2 d}{d+2}}(|t| \leq T)} \\
& +\left\|\left[P_{\leq \tilde{N}_{n}^{\prime} N_{n}} F\left(v_{n}\right)-F\left(P_{\leq \tilde{N}_{n}^{\prime} N_{n}} v_{n}\right)\right] \nabla \chi_{n}\right\|_{L_{t}^{2} L_{x}^{\frac{2 d}{d+2}}\left(|t| \leq T N_{n}^{-2}\right)} .
\end{aligned}
$$

By Lemma 5.2, the first term disappears in the limit as $n \rightarrow \infty$. That lemma also applies to the second term after a change of variables to give

$$
\begin{aligned}
& \left\|\left[P_{\leq \tilde{N}_{n}^{\prime} N_{n}} F\left(v_{n}\right)-F\left(P_{\leq \tilde{N}_{n}^{\prime} N_{n}} v_{n}\right)\right] \nabla \chi_{n}\right\|_{L_{t}^{2} \frac{2 d}{d+2}}\left(|t| \leq T N_{n}^{-2}\right) \\
& \lesssim N_{n}^{\prime}\left\|P_{\leq \tilde{N}_{n}^{\prime} N_{n}} F\left(v_{n}\right)-F\left(P_{\leq \tilde{N}_{n}^{\prime} N_{n}} v_{n}\right)\right\|_{L_{t}^{2} L_{x}^{\frac{2 d}{d+2}}\left(|t| \leq T N_{n}^{-2}\right)} \rightarrow 0 \text { as } n \rightarrow \infty . \\
& \lesssim \frac{N_{n}^{\prime}}{N_{n}}\left\|P_{\leq \tilde{N}_{n}^{\prime}} F(v)-F\left(P_{\leq \tilde{N}_{n}^{\prime}} v\right)\right\|_{L_{t}^{2} L_{x}^{\frac{2 d}{d+2}}(|t| \leq T)} \rightarrow 0 \text {. }
\end{aligned}
$$

Therefore

$$
\lim _{n \rightarrow \infty}\left\|\nabla\left(a^{\prime}\right)\right\|_{L_{t}^{2} L_{x}^{\frac{2 d}{d+2}}\left(|t| \leq T N_{n}^{-2}\right)}=0 .
$$

By changing variables, using the bound $\left|x_{n}\right| \lesssim N_{n}$, and referring to Lemma 5.2 once more,

$$
\begin{aligned}
& \left\||x|\left(a^{\prime}\right)\right\|_{L_{t}^{2} L_{x}^{\frac{2 d}{d+2}}} \lesssim N_{n}\left\|P_{\leq \tilde{N}_{n}^{\prime} N_{n}} F\left(v_{n}\right)-F\left(P_{\leq \tilde{N}_{n}^{\prime} N_{n}} v_{n}\right)\right\|_{L_{t}^{2} L_{x}^{\frac{2 d}{d+2}}\left(|t| \leq T N_{n}^{-2}\right)} \\
& \lesssim\left\|P_{\leq \tilde{N}_{n}^{\prime}} F(v)-F\left(P_{\leq \tilde{N}_{n}^{\prime}} v\right)\right\|_{L_{t}^{2} L_{x}^{\frac{2 d}{d+2}}(|t| \leq T)} \rightarrow 0 \text { as } n \rightarrow \infty .
\end{aligned}
$$

It follows from Lemma 2.2 that

$$
\lim _{n \rightarrow \infty}\left\|H^{\frac{1}{2}}\left(a^{\prime}\right)\right\|_{L_{t}^{2} L_{x}^{\frac{2 d}{d+2}}\left(|t| \leq T N_{n}^{-2}\right)}=0 .
$$

To estimate $\left(a^{\prime \prime}\right)$, we use the Leibniz rule, a change of variables, Hölder, Sobolev embedding, the bound (5.2), and dominated convergence to obtain

$$
\begin{aligned}
& \left\|\nabla\left(a^{\prime \prime}\right)\right\|_{L_{t}^{2} L_{x}^{\frac{2 d}{d+2}}} \lesssim\left\|\left|P_{\leq \tilde{N}_{n} N_{n}} v_{n}\right|^{\frac{4}{d-2}} \nabla P_{\leq \tilde{N}_{n}^{\prime} N_{n}} v_{n}\right\|_{L_{t}^{2} L_{x}^{\frac{2 d}{d+2}}\left(|t| \leq T N_{n}^{-2},\left|x-x_{n}\right| \sim\left(N_{n}^{\prime}\right)^{-1}\right)} \\
& +\frac{N_{n}^{\prime}}{N_{n}}\left\|P_{\leq \tilde{N}_{n}^{\prime} N_{n}} v_{n}\right\|_{L_{t}^{\infty} L_{x}^{\frac{d+2}{d-2}}}^{\frac{2 d}{d-2}} \\
& \lesssim\|\nabla v\|_{L_{t}^{\frac{2(d+2)}{d-2}}} \frac{2 d(d+2)}{L_{x}^{\frac{d}{d^{2}+4}}}\left\|P_{\leq \tilde{N}_{n}^{\prime}} v\right\|_{L_{t, x}^{\frac{2(d-2)}{d-2}}\left(|t| \leq T,|x| \sim \frac{N_{n}}{N_{n}^{n}}\right)}+O\left(\frac{N_{n}^{\prime}}{N_{n}}\right) \\
& \lesssim C(E(v))\left(\left\|P_{>\tilde{N}_{n}^{\prime}} v\right\|_{L_{t, x}^{\frac{2(d+2)}{d-2}}}+\|v\|_{L_{t, x}^{\frac{2(d+2)}{d-2}}}\left(|t| \leq T,|x| \gtrsim \frac{N_{n}}{\left.N_{n}^{\prime}\right)}\right.\right. \\
& =o(1)+O\left(\frac{N_{n}^{\prime}}{N_{n}}\right) .
\end{aligned}
$$


Similarly,

$$
\begin{aligned}
& \left\||x|\left(a^{\prime \prime}\right)\right\|_{L_{t}^{2} L_{x}^{\frac{2 d}{d+2}}} \sim\left\|F\left(P_{\leq \tilde{N}_{n}^{\prime}} v\right)\right\|_{L_{t}^{\frac{2(d+2)}{d-2}}} L_{x}^{\frac{2 d}{d-2}}\left(|t| \leq T,|x| \sim \frac{N_{n}}{N_{n}^{\prime}}\right) \\
& \lesssim\left(\left\|P_{>\tilde{N}_{n}^{\prime}} v\right\|_{L_{t}^{\frac{2(d+2)}{d-2}}} L_{x}^{\frac{2 d}{d-2}}(|t| \leq T)\right. \\
& =o(1) .
\end{aligned}
$$

Therefore

$$
\lim _{N \rightarrow \infty}\left\|H^{\frac{1}{2}}\left(a^{\prime \prime}\right)\right\|_{L_{t}^{2} L_{t, x}^{\frac{2 d}{d+2}}\left(|t| \leq T N_{n}^{-2}\right)}=0
$$

as well. This completes the analysis for $(a)$.

The estimates for $(b),(c),(d)$ are less involved. For $(b)$, note that on the support of the function we have $\left.|| x_{n}\right|^{2}-|x|^{2}|=| x_{n}-x|| x_{n}+x \mid \sim N_{n}\left(N_{n}^{\prime}\right)^{-1}$. Thus by Hölder and Sobolev embedding,

$$
\begin{aligned}
& \|\nabla(b)\|_{L_{t}^{1} L_{x}^{2}\left(|t| \leq T N_{n}^{-2}\right)} \\
& \lesssim \frac{N_{n}}{N_{n}^{\prime}}\left\|\nabla P_{\leq \tilde{N}_{n}^{\prime} N_{n}} v_{n}\right\|_{L_{t}^{1} L_{x}^{2}\left(|t| \leq T N_{n}^{-2}\right)}+N_{n}\left\|P_{\leq \tilde{N}_{n}^{\prime} N_{n}} v_{n}\right\|_{L_{t}^{1} L_{x}^{2}\left(|t| \leq T N_{n}^{-2},\left|x-x_{n}\right| \sim\left(N_{n}^{\prime}\right)^{-1}\right)} \\
& \lesssim\left(N_{n}^{\prime} N_{n}\right)^{-1}\left\|\nabla v_{n}\right\|_{L_{t}^{\infty} L_{x}^{2}} \rightarrow 0 \text { as } n \rightarrow \infty .
\end{aligned}
$$

Using Hölder and Sobolev embedding, we have

$$
\begin{aligned}
\||x|(b)\|_{L_{t}^{1} L_{x}^{2}\left(|t| \leq T N_{n}^{-2}\right)} & \sim \frac{N_{n}^{2}}{N_{n}^{\prime}}\left\|P_{\leq \tilde{N}_{n}^{\prime} N_{n}} v_{n}\right\|_{L_{t}^{1} L_{x}^{2}\left(|t| \leq T N_{n}^{-2},\left|x-x_{n}\right| \lesssim\left(N_{n}^{\prime}\right)^{-1}\right)} \\
& \lesssim\left\{\begin{array}{cc}
\left(N_{n}^{\prime}\right)^{-2}\left\|\nabla v_{n}\right\|_{L_{t}^{\infty} L_{x}^{2}}, \quad \lim _{n \rightarrow \infty} N_{n}^{-1}\left|x_{n}\right|=0 \\
\left\|v_{n}\right\|_{L_{t}^{\infty} L_{x}^{2}}=O\left(N_{n}^{-1}\right), \quad \lim _{n \rightarrow \infty} N_{n}^{-1}\left|x_{n}\right|>0,
\end{array}\right.
\end{aligned}
$$

which vanishes as $n \rightarrow \infty$ in either case. Thus $\left\|H^{1 / 2}(b)\right\|_{L_{t}^{1} L_{x}^{2}} \rightarrow 0$. The term $(c)$ is dealt with similarly. For (d), use Hölder, Bernstein, and the definition (5.7) of the frequency cutoffs $\tilde{N}_{n}^{\prime}$ to obtain

$$
\begin{aligned}
\|\nabla(d)\|_{L_{t}^{1} L_{x}^{2}\left(|t| \leq T N_{n}^{-2}\right)} & \lesssim N_{n}^{\prime}\left\||\nabla|^{2} P_{\leq \tilde{N}_{n}^{\prime} N_{n}} v_{n}\right\|_{L_{t}^{1} L_{x}^{2}}+\left\|\left|\nabla P_{\leq \tilde{N}_{n}^{\prime} N_{n}} v_{n}\right|\left(|\nabla|^{2} \chi_{n}\right)\right\|_{L_{t}^{1} L_{x}^{2}} \\
& \lesssim\left[\left(\frac{N_{n}^{\prime}}{N_{n}}\right)^{\frac{1}{2}}+\left(\frac{N_{n}^{\prime}}{N_{n}}\right)^{2}\right]\left\|\nabla v_{n}\right\|_{L_{t}^{\infty} L_{x}^{2}} \rightarrow 0 .
\end{aligned}
$$

Applying Hölder in the time variable, we get

$$
\||x|(d)\|_{L_{t}^{1} L_{x}^{2}\left(|t| \leq T N_{n}^{-2}\right)} \lesssim \frac{N_{n}^{\prime}}{N_{n}}\left\|\nabla v_{n}\right\|_{L_{t}^{\infty} L_{x}^{2}} \rightarrow 0 .
$$

This completes the proof of the lemma.

Next, we estimate the error over the time intervals $\left[-2, T N_{n}^{-2}\right]$ and $\left[T N_{n}^{-2}, 2\right]$.

Lemma 5.4. $\lim _{T \rightarrow \infty} \lim \sup _{n \rightarrow \infty}\left\|H^{\frac{1}{2}} e_{n}\right\|_{N\left(\left[-2, T N_{n}^{-2}\right] \cup\left[T N_{n}^{-2}, 2\right]\right)}=0$.

Proof. We consider just the forward time interval as the other interval is treated similarly. Since $\tilde{v}_{n}^{T}$ solves the linear equation, the error $e_{n}$ is just the nonlinear term:

$$
e_{n}=\left(i \partial_{t}-H\right) \tilde{v}_{n}^{T}-F\left(\tilde{v}_{n}^{T}\right)=-F\left(\tilde{v}_{n}^{T}\right) .
$$

By the chain rule (Corollary 2.4) and Strichartz,

$$
\left\|H^{\frac{1}{2}} e_{n}\right\|_{N\left(\left[T N_{n}^{-2}, 2\right]\right)} \lesssim\left\|\tilde{v}_{n}^{T}\right\|_{L_{t, x}^{\frac{2(d-2)}{d-2}}\left(\left[T N_{n}^{-2}, 2\right]\right)}^{\frac{4}{d-2}}\left\|\tilde{v}_{n}^{T}\left(T N_{n}^{-2}\right)\right\|_{\Sigma} .
$$


By definition $\tilde{v}_{n}^{T}\left(T N_{n}^{-2}\right)=e^{-\frac{i T N_{n}^{-2}\left|x_{n}\right|^{2}}{2}} \tilde{G}_{n} S_{n} P_{\leq \tilde{N}_{n}^{\prime}} v\left(T N_{n}^{-2}-t_{n}\right)$, so Lemma 4.2 implies that



is bounded in either case. Using Strichartz and interpolation, it suffices to show

$$
\lim _{T \rightarrow \infty} \limsup _{n \rightarrow \infty}\left\|\tilde{v}_{n}^{T}\right\|_{L_{T}^{\infty} L_{x}^{\frac{2 d}{d-2}}\left(\left[T N_{n}^{-2}, 2\right]\right)}=0 .
$$

As we are assuming Conjecture 1.1, there exists $v_{\infty} \in \dot{H}^{1}$ so that

$$
\lim _{t \rightarrow \infty}\left\|v(t)-e^{\frac{i t \Delta}{2}} v_{\infty}\right\|_{\dot{H}_{x}^{1}}=0 .
$$

Then one also has

$$
\lim _{t \rightarrow \infty} \limsup _{n \rightarrow \infty}\left\|P_{\leq \tilde{N}_{n}^{\prime}} v(t)-e^{\frac{i t \Delta}{2}} v_{\infty}\right\|_{\dot{H}_{x}^{1}}=0
$$

and Lemma 4.2 implies that

$$
\lim _{T \rightarrow \infty} \limsup _{n \rightarrow \infty}\left\|\tilde{v}_{n}\left(T N_{n}^{-2}\right)-e^{-\frac{i T N_{n}^{-2}\left|x_{n}\right|^{2}}{2}} G_{n} S_{n}\left(e^{\frac{i T \Delta}{2}} v_{\infty}\right)\right\|_{\Sigma}=0 .
$$

An application of Strichartz and Corollary 4.9 yields

$$
\begin{aligned}
\tilde{v}_{n}(t) & =e^{-i\left(t-T N_{n}^{-2}\right) H}\left[\tilde{v}_{n}\left(T N_{n}^{-2}\right)\right] \\
& =e^{-i\left(t-T N_{n}^{-2}\right) H}\left[e^{-\frac{i T N_{n}^{-2}\left|x_{n}\right|^{2}}{2}} G_{n} S_{n} e^{\frac{i T \Delta}{2}} v_{\infty}\right]+\text { error } \\
& =e^{-i t H}\left[G_{n} S_{n} v_{\infty}\right]+\text { error }
\end{aligned}
$$

where $\lim _{T \rightarrow \infty} \lim \sup _{n \rightarrow \infty}\|\operatorname{error}\|_{\Sigma}=0$ uniformly in $t$. By Sobolev embedding,

$$
\begin{aligned}
& \lim _{T \rightarrow \infty} \limsup _{n \rightarrow \infty}\left\|\tilde{v}_{n}\right\|_{L_{t}^{\infty} L_{x}^{\frac{2 d}{d-2}}\left(\left[T N_{n}^{-2}, 2\right]\right)} \\
& =\lim _{T \rightarrow \infty} \limsup _{n \rightarrow \infty}\left\|e^{-i t H}\left[G_{n} S_{n} v_{\infty}\right]\right\|{ }_{L_{t}^{\infty} L_{x}^{\frac{2 d}{d-2}}\left(\left[T N_{n}^{-2}, 2\right]\right)} .
\end{aligned}
$$

A standard density argument using the dispersive estimate for $e^{-i t H}$ shows that the last limit is zero.

Lemmas 5.3 and 5.4 together establish (5.11).

Lemma 5.5 (Matching initial data). Let $u_{n}(0)=e^{i t_{n} H} G_{n} S_{n} \phi$ as in Proposition 5.1. Then

$$
\lim _{T \rightarrow \infty} \limsup _{n \rightarrow \infty}\left\|\tilde{v}_{n}^{T}\left(-t_{n}\right)-u_{n}(0)\right\|_{\Sigma}=0 .
$$

Proof. If $t_{n} \equiv 0$, then by definition $\tilde{v}_{n}^{T}(0)=G_{n} S_{n} P_{\leq N_{n}^{\prime}} \phi$, so Lemma 4.2 and the definition (5.7) of the frequency parameter $N_{n}^{\prime}$ imply

$$
\lim _{n \rightarrow \infty}\left\|\tilde{v}_{n}^{T}(0)-u_{n}(0)\right\|_{\Sigma} \lesssim \lim _{n \rightarrow \infty}\left\{\begin{array}{ll}
\left\|P_{>N_{n}^{\prime}} \phi\right\|_{H^{1}}, & \lim _{n \rightarrow \infty} N_{n}^{-1}\left|x_{n}\right|>0 \\
\left\|P_{>N_{n}^{\prime}} \phi\right\|_{\dot{H}^{1}}, & \lim _{n \rightarrow \infty} N_{n}^{-1}\left|x_{n}\right|=0
\end{array}\right\}=0
$$

Next we consider the case $N_{n}^{2} t_{n} \rightarrow \infty$; the case $N_{n}^{2} t_{n} \rightarrow-\infty$ works similarly. Arguing as in the previous lemma and recalling that in this case, the solution $v$ was chosen to scatter backward in time to $e^{\frac{i t \Delta}{2}} \phi$, for $n$ large we have

$$
\tilde{v}_{n}^{T}\left(-t_{n}\right)=e^{i t_{n} H}\left[G_{n} S_{n} \phi\right]+\text { error }
$$

where $\lim _{T \rightarrow \infty} \lim \sup _{n \rightarrow \infty} \|$ error $\|_{\Sigma} \rightarrow 0$. The claim follows. 
For each fixed $T>0$, set

$$
\tilde{u}_{n}^{T}(t)=\tilde{v}_{n}^{T}\left(t-t_{n}\right)
$$

which is defined for $t \in[-1,1]$. Then for a fixed large value of $T$, this is an approximate solution for all $n$ sufficiently large in the sense of Proposition 3.3. Indeed, by (5.9) and (5.10), $\tilde{u}_{n}^{T}$ satisfy the hypotheses (3.1) with $E \lesssim\|\phi\|_{\dot{H}^{1}}$ and $L=C\left(\|\phi\|_{\dot{H}^{1}}\right)$. Lemmas 5.3, 5.4, 5.5, Sobolev embedding, and Strichartz show that for any $\varepsilon>0$, there exists $T>0$ so that $\tilde{u}_{n}^{T}$ satisfies the hypotheses (3.2) for all large $n$. Invoking Proposition 3.3 we obtain the first claim of Proposition 5.1 concerning the existence of solutions

The remaining assertion of Proposition 5.1 regarding approximation by smooth functions will follow from the next lemma. Recall that we use the notation

$$
\|f\|_{L_{t}^{q} \Sigma_{x}^{r}}=\left\|H^{\frac{1}{2}} f\right\|_{L_{t}^{q} L_{x}^{r}} .
$$

Lemma 5.6. Fix finitely many admissible $\left(q_{k}, r_{k}\right)$ with $2 \leq r_{k}<d$. For every $\varepsilon>0$, there exists a smooth function $\psi^{\varepsilon} \in C_{c}^{\infty}\left(\mathbf{R} \times \mathbf{R}^{d}\right)$ such that for all $k$

$$
\limsup _{T \rightarrow \infty} \limsup _{n \rightarrow \infty}\left\|\tilde{v}_{n}^{T}-\tilde{G}_{n}\left[e^{-\frac{i t N_{n}^{-2}\left|x_{n}\right|^{2}}{2}} \psi^{\varepsilon}\right]\left(t+t_{n}\right)\right\|_{L_{T}^{q_{k}} \Sigma_{x}^{r}([-2,2])}<\varepsilon .
$$

Proof. We continue using the notation defined at the beginning. Let

$$
\tilde{w}_{n}^{T}=\left\{\begin{array}{cc}
e^{-\frac{i t\left|x_{n}\right|^{2}}{2}} \tilde{G}_{n}\left[S_{n} v\right]\left(t+t_{n}\right), & |t| \leq T N_{n}^{-2} \\
e^{-i\left(t-T N_{n}^{-2}\right) H}\left[\tilde{w}_{n}^{T}\left(T N_{n}^{-2}\right)\right], & t \geq T N_{n}^{-2} \\
e^{-i\left(t+T N_{n}^{-2}\right) H}\left[\tilde{w}_{n}^{T}\left(-T N_{n}^{-2}\right)\right], & t \leq-T N_{n}^{-2}
\end{array}\right.
$$

This is essentially $\tilde{v}_{n}^{T}$ in (5.8) without the frequency cutoffs. We observe first that $\tilde{v}_{n}^{T}$ can be well-approximated by $\tilde{w}_{n}^{T}$ in spacetime:

$$
\begin{aligned}
& \limsup _{n \rightarrow \infty}\left\|\tilde{v}_{n}^{T}-\tilde{w}_{n}^{T}\right\|_{L_{t}^{q_{k}} \Sigma_{x}^{r_{k}}([-2,2])}=0, \\
& \sup _{T>0} \limsup _{n \rightarrow \infty}\left\|\tilde{w}_{n}^{T}\right\|_{L_{t}^{q_{k}} \Sigma_{x}^{r_{k}}([-2,2])}<\infty .
\end{aligned}
$$

Indeed by dominated convergence,

$$
\left\|\nabla\left(v-P_{\leq \tilde{N}_{n}^{\prime}} v\right)\right\|_{L_{t}^{q_{k}} L_{x}^{r_{k}}\left(\mathbf{R} \times \mathbf{R}^{d}\right)} \rightarrow 0 \text { as } n \rightarrow \infty,
$$

thus (5.14) follows from Lemma 4.2 and the Strichartz inequality for $e^{-i t H}$.

The next observation is that most of the spacetime norm of $\tilde{w}_{n}^{T}$ is concentrated in the time interval $|t| \leq T N_{n}^{-2}$ :

$$
\lim _{T \rightarrow \infty} \limsup _{n \rightarrow \infty}\left\|\tilde{w}_{n}^{T}\right\|_{L_{t}^{q_{k}} \Sigma_{x}^{r_{k}}\left(\left[-2,-T N_{n}^{-2}\right] \cup\left[T N_{n}^{-2}, 2\right]\right)}=0 .
$$

To see this, it suffices by symmetry to consider the forward interval. Recall that $v$ scatters forward in $\dot{H}^{1}$ to some $e^{\frac{i t \Delta}{2}} v_{\infty}$. By Lemma 4.2,

$$
\lim _{T \rightarrow \infty} \limsup _{n \rightarrow \infty} \|\left(\tilde{G}_{n} S_{n} v\left(T N_{n}^{-2}-t_{n}\right)-G_{n} S_{n}\left(e^{\frac{i T \Delta}{2}} v_{\infty}\right) \|_{\Sigma}=0 .\right.
$$

By Strichartz,

$\lim _{T \rightarrow \infty} \limsup _{n \rightarrow \infty}\left\|e^{\frac{i T N_{n}^{-2}\left|x_{n}\right|^{2}}{2}} \tilde{w}_{n}^{T}-e^{-i\left(t-T N_{n}^{-2}\right) H}\left[G_{n} S_{n}\left(e^{\frac{i T \Delta}{2}} v_{\infty}\right)\right]\right\|_{L_{t}^{q_{k}} \sum_{x}^{r_{k}}\left(\left[T N_{n}^{-2}, 2\right]\right)}=0$

By Corollary 4.9 and Strichartz, for each $T>0$ we have

$\limsup _{n \rightarrow \infty}\left\|e^{-i\left(t-T N_{n}^{-2}\right) H}\left[G_{n} S_{n}\left(e^{\frac{i T \Delta}{2}} v_{\infty}\right)\right]-e^{\frac{i T\left(r_{\infty}\right)^{2}}{2}} e^{-i t H}\left[G_{n} S_{n} v_{\infty}\right]\right\|_{L_{t}^{q_{k}} \Sigma_{x}^{r_{k}}\left(\left[T N_{n}^{-2}, 2\right]\right)}=0$. 
For each $\varepsilon>0$, choose $v_{\infty}^{\varepsilon} \in C_{c}^{\infty}$ such that $\left\|v_{\infty}-v_{\infty}^{\varepsilon}\right\|_{\dot{H}^{1}}<\varepsilon$. By the dispersive estimate,

$$
\left\|e^{-i t H}\left[G_{n} v_{\infty}^{\varepsilon}\right]\right\|_{L_{t}^{q_{k}} L_{x}^{r_{k}}\left(\left[T N_{n}^{-2}, 2\right]\right)} \lesssim T^{-\frac{1}{q_{k}}}\left\|v_{\infty}^{\varepsilon}\right\|_{L_{x}^{r_{k}^{\prime}}}
$$

Combining the above with Strichartz and Lemma 4.2 we get

$$
\limsup _{n \rightarrow \infty}\left\|\tilde{w}_{n}^{T}\right\|_{L_{t}^{q_{k}} \sum_{x}^{r_{k}}\left(\left[T N_{n}^{-2}, 2\right]\right)} \lesssim o(1)+\varepsilon+O_{\varepsilon, q_{k}}\left(T^{-\frac{1}{q_{k}}}\right) \text { as } T \rightarrow \infty .
$$

Taking $T \rightarrow \infty$, we find

$$
\limsup _{T \rightarrow \infty} \limsup _{n \rightarrow \infty}\left\|\tilde{w}_{n}^{T}\right\|_{L_{t}^{q_{k}} \sum_{x}^{r}{ }^{r}\left(\left[T N_{n}^{-2}, 2\right]\right)} \lesssim \varepsilon
$$

for any $\varepsilon>0$, thereby establishing (5.15).

Choose $\psi^{\varepsilon} \in C_{c}^{\infty}\left(\mathbf{R} \times \mathbf{R}^{d}\right)$ such that $\sum_{k=1}^{N}\left\|v-\psi^{\varepsilon}\right\|_{L_{t}^{q_{k}} \dot{H}_{x}^{1, r_{k}}}<\varepsilon$. By combining Lemma 4.2 with (5.14) and (5.15), we get

$$
\lim _{T \rightarrow \infty} \limsup _{n \rightarrow \infty}\left\|\tilde{v}_{n}(t, x)-e^{-\frac{i t\left|x_{n}\right|^{2}}{2}} \tilde{G}_{n} \psi^{\varepsilon}\left(t+t_{n}\right)\right\|_{L_{t}^{q_{k}} \sum_{x}^{r_{k}}([-2,2])} \lesssim \varepsilon .
$$

This completes the proof of the lemma, hence Proposition 5.1 .

Remark. From the proof it is clear that that the proposition also holds if the interval $I=[-1,1]$ is replaced by any smaller interval.

\section{Palais-Smale and the Proof of Theorem 1.2}

In this section we prove a Palais-Smale condition on sequences of blowing up solutions to (1.1). This will quickly lead to the proof of Theorem 1.2,

For a maximal solution $u$ to (1.1), define

$$
S_{*}(u, L)=\sup \left\{S_{I}(u): I \text { is an open interval with } \leq L\right\},
$$

where we set $S_{I}(u)=\infty$ if $u$ is not defined on $I$. All solutions in this section are assumed to be maximal. By the triangle inequality, finiteness of $S_{*}(u, L)$ for some $L$ implies finiteness for all $L$. Set

$$
\begin{gathered}
\Lambda_{d}(E, L)=\sup \left\{S_{*}(u, L): u \text { solves (1.1), } \mu=+1, E(u)=E\right\} \\
\Lambda_{f}(E, L)=\sup \left\{S_{*}(u, L): u \text { solves (1.1), } \mu=-1, E(u)=E,\right. \\
\left.\|\nabla u(0)\|_{L^{2}}<\|\nabla W\|_{L^{2}}\right\} .
\end{gathered}
$$

Note as before that finiteness for some $L$ is equivalent to finiteness for all $L$. Finally, define

$$
\begin{aligned}
\Lambda_{d}(E) & =\lim _{L \rightarrow 0} \Lambda_{d}(E, L), \quad \Lambda_{f}(E)=\lim _{L \rightarrow 0} \Lambda_{f}(E, L), \\
\mathcal{E}_{d} & =\left\{E: \Lambda_{d}(E)<\infty\right\}, \quad \mathcal{E}_{f}=\left\{E: \Lambda_{f}(E)<\infty\right\} .
\end{aligned}
$$

By the local theory, Theorem 1.2 is equivalent to the assertions

$$
\mathcal{E}_{d}=[0, \infty), \quad \mathcal{E}_{f}=\left[0, E_{\Delta}(W)\right) .
$$

Suppose Theorem 1.2 failed. By the small data theory, $\mathcal{E}_{d}, \mathcal{E}_{f}$ are nonempty and open, and the failure of Theorem 1.2 implies the existence of a critical energy $E_{c}>0$, with $E_{c}<E_{\Delta}(W)$ in the focusing case such that $\Lambda_{d}(E), \Lambda_{f}(E)=\infty$ for $E>E_{c}$ and $\Lambda_{d}(E), \Lambda_{f}(E)<\infty$ for all $E<E_{c}$. 
Define the spaces

$$
\dot{X}^{1}=\left\{\begin{array}{cc}
L_{t, x}^{10} \cap L_{t}^{5} \sum_{x}^{\frac{30}{11}}\left(\left[-\frac{1}{2}, \frac{1}{2}\right] \times \mathbf{R}^{d}\right), & d=3 \\
L_{t, x}^{\frac{2(d+2)}{d-2}} \cap L_{t}^{\frac{2(d+2)}{d}} \Sigma_{x}^{\frac{2(d+2)}{d}}\left(\left[-\frac{1}{2}, \frac{1}{2}\right] \times \mathbf{R}^{d}\right), & d \geq 4 .
\end{array}\right.
$$

When $d=3$, we also define

$$
\dot{Y}^{1}=\dot{X}^{1} \cap L_{t}^{\frac{10}{3}} \Sigma_{x}^{\frac{10}{3}}\left(\left[-\frac{1}{2}, \frac{1}{2}\right] \times \mathbf{R}^{3}\right) .
$$

Proposition 6.1 (Palais-Smale). Assume Conjecture 1.1 holds. Suppose that $u_{n}$ : $\left(t_{n}-\frac{1}{2}, t_{n}+\frac{1}{2}\right) \times \mathbf{R}^{d} \rightarrow \mathbf{C}$ is a sequence of solutions with

$$
\lim _{n \rightarrow \infty} E\left(u_{n}\right)=E_{c}, \lim _{n \rightarrow \infty} S_{\left(t_{n}-\frac{1}{2}, t_{n}\right]}\left(u_{n}\right)=\lim _{n \rightarrow \infty} S_{\left[t_{n}, t_{n}+\frac{1}{2}\right)}\left(u_{n}\right)=\infty .
$$

In the focusing case, assume also that $E_{c}<E_{\Delta}(W)$ and $\left\|\nabla u_{n}\left(t_{n}\right)\right\|_{L^{2}}<\|\nabla W\|_{L^{2}}$. Then there exists a subsequence such that $u_{n}\left(t_{n}\right)$ converges in $\Sigma$.

Proof of Proposition 6.1. By replacing $u_{n}(t)$ with $u_{n}\left(t+t_{n}\right)$, we may assume $t_{n} \equiv 0$. Note that by energy conservation and Corollary 7.2, this time translation does not change the hypotheses of the focusing case.

Observe (referring to the discussion in Section 7 for the focusing case) that the sequence $u_{n}(0)$ is bounded in $\Sigma$. Applying Proposition 4.14 after passing to a subsequence we have a decomposition

$$
u_{n}(0)=\sum_{j=1}^{J} e^{i t_{n}^{j} H} G_{n} S_{n} \phi^{j}+w_{n}^{J}=\sum_{j=1}^{J} \phi_{n}^{j}+w_{n}^{J}
$$

with the properties stated in that proposition. In particular, the remainder has asymptotically trivial linear evolution:

$$
\lim _{J \rightarrow J^{*}} \limsup _{n \rightarrow \infty}\left\|e^{-i t H} w_{n}^{J}\right\|_{L_{t, x}^{\frac{2(d+2)}{d-2}},}
$$

and we have asymptotic decoupling of energy:

$$
\sup _{J} \lim _{n \rightarrow \infty}\left|E\left(u_{n}\right)-\sum_{j=1}^{J} E\left(\phi_{n}^{j}\right)-E\left(w_{n}^{J}\right)\right|=0 .
$$

Observe that $\liminf _{n} E\left(\phi_{n}^{j}\right) \geq 0$. This is obvious in the defocusing case. In the focusing case, (4.15) and the discussion in Section 7 imply that

$$
\sup _{j} \limsup _{n}\left\|\phi_{n}^{j}\right\|_{\Sigma} \leq\left\|u_{n}\right\|_{\Sigma}<\|\nabla W\|_{L^{2}}
$$

so the claim follows from Lemma 7.1. Therefore, there are two possibilities.

Case 1: $\sup _{j} \lim \sup _{n \rightarrow \infty} E\left(\phi_{n}^{j}\right)=E_{c}$.

By combining (6.2) with the fact that the profiles $\phi_{n}^{j}$ are nontrivial in $\Sigma$, we deduce that $J^{*}=1$ and

$$
u_{n}(0)=e^{i t_{n} H} G_{n} S_{n} \phi+w_{n}, \lim _{n \rightarrow \infty}\left\|w_{n}\right\|_{\Sigma}=0 .
$$

We will show that $N_{n} \equiv 1$ (thus $x_{n}=0$ and $t_{n}=0$ ). Suppose $N_{n} \rightarrow \infty$.

Proposition 5.1 implies that for all large $n$, there exists a unique solution $u_{n}$ on $\left[-\frac{1}{2}, \frac{1}{2}\right]$ with $u_{n}(0)=e^{i t_{n} H} G_{n} S_{n} \phi$ and $\lim \sup _{n \rightarrow \infty} S_{\left(-\frac{1}{2}, \frac{1}{2}\right)}\left(u_{n}\right) \leq C\left(E_{c}\right)$. By perturbation theory (Proposition 3.3),

$$
\limsup _{n \rightarrow \infty} S_{\left[-\frac{1}{2}, \frac{1}{2}\right]}\left(u_{n}\right) \leq C\left(E_{c}\right),
$$


which is a contradiction. Therefore, $N_{n} \equiv 1, t_{n}^{j} \equiv 0, x_{n}^{j} \equiv 0$, and

$$
u_{n}(0)=\phi+w_{n}
$$

for some $\phi \in \Sigma$. This is the desired conclusion.

Case 2: $\sup _{j} \lim \sup _{n \rightarrow \infty} E\left(\phi_{n}^{j}\right) \leq E_{c}-2 \delta$ for some $\delta>0$.

By the definition of $E_{c}$, there exist solutions $v_{n}^{j}:\left(-\frac{1}{2}, \frac{1}{2}\right) \times \mathbf{R}^{d} \rightarrow \mathbf{C}$ with

$$
\left\|v_{n}^{j}\right\|_{L_{t, x}^{\frac{2(d+2)}{d-2}}\left(\left[-\frac{1}{2}, \frac{1}{2}\right]\right)} \lesssim_{E_{c}, \delta} E\left(\phi_{n}^{j}\right)^{\frac{1}{2}} .
$$

By standard arguments (c.f. [29, Lemma 3.11]), this implies the seemingly stronger bound

$$
\left\|v_{n}^{j}\right\|_{\dot{X}^{1}} \lesssim E_{c}, \delta E\left(\phi_{n}^{j}\right)^{\frac{1}{2}}
$$

In the case $d=3$, we also have $\left\|v_{n}^{j}\right\|_{\dot{Y}^{1}} \lesssim E\left(\phi_{n}^{j}\right)^{\frac{1}{2}}$. Put

$$
u_{n}^{J}=\sum_{j=1}^{J} v_{n}^{j}+e^{-i t H} w_{n}^{J} .
$$

We claim that for sufficiently large $J$ and $n, u_{n}^{J}$ is an approximate solution in the sense of Proposition 3.3 To prove this claim, we check that $u_{n}^{J}$ has the following three properties:

(i) $\lim _{J \rightarrow J^{*}} \lim \sup _{n \rightarrow \infty}\left\|u_{n}^{J}(0)-u_{n}(0)\right\|_{\Sigma}=0$.



(iii) $\lim _{J \rightarrow J^{*}} \lim \sup _{n \rightarrow \infty}\left\|H^{\frac{1}{2}} e_{n}^{J}\right\|_{N\left(\left[-\frac{1}{2}, \frac{1}{2}\right]\right)}=0$, where

$$
e_{n}=\left(i \partial_{t}-H\right) u_{n}^{J}-F\left(u_{n}^{J}\right)
$$

There is nothing to check for part (i) as $u_{n}^{J}(0)=u_{n}(0)$ by construction. The verification of (ii) relies on the asymptotic decoupling of the nonlinear profiles $v_{n}^{j}$, which we record in the following two lemmas.

Lemma 6.2 (Orthogonality). Suppose that two frames $\mathcal{F}^{j}=\left(t_{n}^{j}, x_{n}^{j}, N_{n}^{j}\right), \mathcal{F}^{k}=$ $\left(t^{k}, x_{n}^{k}, N_{n}^{k}\right)$ are orthogonal, and let $\tilde{G}_{n}^{j}, \tilde{G}_{n}^{k}$ be the associated spacetime scaling and translation operators as defined in (4.1). Then for all $\psi^{j}, \psi^{k}$ in $C_{c}^{\infty}\left(\mathbf{R} \times \mathbf{R}^{d}\right)$,

$$
\begin{aligned}
& \left\|\left(\tilde{G}_{n}^{j} \psi^{j}\right)\left(\tilde{G}_{n}^{k} \psi^{k}\right)\right\|_{L_{t, x}^{\frac{d+2}{d-2}}}+\left\|\left(\tilde{G}_{n}^{j} \psi^{j}\right) \nabla\left(\tilde{G}_{n}^{k} \psi^{k}\right)\right\|_{L_{t, x}^{\frac{d+2}{d-1}}}+\left\||x|\left(\tilde{G}_{n}^{j} \psi^{j}\right)\left(\tilde{G}_{n}^{k} \psi^{k}\right)\right\|_{L_{t, x}^{\frac{d+2}{d-1}}} \\
& +\left\||x|^{2}\left(\tilde{G}_{n}^{j} \psi^{j}\right)\left(\tilde{G}_{n}^{k} \psi^{k}\right)\right\|_{L_{t, x} \frac{d+2}{t, x}}+\left\|\left(\nabla \tilde{G}_{n}^{j} \psi^{j}\right)\left(\nabla \tilde{G}_{n}^{k} \psi^{k}\right)\right\|_{L_{t, x}^{\frac{d+2}{x}}} \rightarrow 0
\end{aligned}
$$

as $n \rightarrow \infty$. When $d=3$, we also have

$$
\left\||x|^{2}\left(\tilde{G}_{n}^{j} \psi^{j}\right)\left(\tilde{G}_{n}^{k} \psi^{k}\right)\right\|_{L_{t}^{5} L_{x}^{\frac{15}{11}}}+\left\|\left(\nabla \tilde{G}_{n}^{j} \psi^{j}\right)\left(\nabla \tilde{G}_{n}^{k} \psi^{k}\right)\right\|_{L_{t}^{5} L_{x}^{\frac{15}{11}}} \rightarrow 0 .
$$

Proof. The arguments for each term are similar, and we only supply the details for the second term. Suppose $N_{n}^{k}\left(N_{n}^{j}\right)^{-1} \rightarrow \infty$. By the chain rule, a change of variables, and Hölder,

$$
\left\|\left(\tilde{G}_{n}^{j} \psi^{j}\right) \nabla\left(\tilde{G}_{n}^{k} \psi^{k}\right)\right\|_{L_{t, x}^{\frac{d+2}{d-1}}}=\left\|\psi^{j} \nabla\left(\tilde{G}_{n}^{j}\right)^{-1} \tilde{G}_{n}^{k} \psi^{k}\right\|_{L_{t, x}^{\frac{d+2}{d-1}}} \leq\left\|\psi^{j} \chi_{n}\right\|_{L_{t, x}^{\frac{2(d+2}{d-2}}}\left\|\nabla \psi^{k}\right\|_{L_{t, x}^{\frac{2(d+2)}{d}}},
$$


where $\chi_{n}$ is the characteristic function of the support of $\nabla\left(\tilde{G}_{n}^{j}\right)^{-1} \tilde{G}_{n}^{k} \psi^{k}$. As the support of $\chi_{n}$ has measure shrinking to zero, we have

$$
\lim _{n \rightarrow \infty}\left\|\psi^{j} \chi_{n}\right\|_{\frac{2(d+2)}{L_{t, x}}}=0
$$

A similar argument deals with the case where $N_{n}^{j}\left(N_{n}^{k}\right)^{-1} \rightarrow \infty$. Therefore, we may suppose that

$$
\frac{N_{n}^{k}}{N_{n}^{j}} \rightarrow N_{\infty} \in(0, \infty)
$$

Make the same change of variables as before, and compute

$$
\nabla\left(\tilde{G}_{n}^{j}\right)^{-1} \tilde{G}_{n}^{k} \psi^{k}(t, x)=\left(\frac{N_{n}^{k}}{N_{n}^{j}}\right)^{\frac{d}{2}}\left(\nabla \psi^{k}\right)\left[\frac{N_{n}^{k}}{N_{n}^{j}} t+\left(N_{n}^{k}\right)^{2}\left(t_{n}^{j}-t_{n}^{k}\right), \frac{N_{n}^{k}}{N_{n}^{j}} x+N_{n}^{k}\left(x_{n}^{j}-x_{n}^{k}\right)\right] .
$$

The decoupling statement (4.17) implies that

$$
\left(N_{n}^{k}\right)^{2}\left(t_{n}^{j}-t_{n}^{k}\right)+N_{n}^{k}\left|x_{n}^{j}-x_{n}^{k}\right| \rightarrow \infty .
$$

Therefore, the supports of $\psi^{j}$ and $\nabla\left(\tilde{G}_{n}^{j}\right)^{-1} \tilde{G}_{n}^{k} \psi^{k}$ are disjoint for large $n$.

Lemma 6.3 (Decoupling of nonlinear profiles). Let $v_{n}^{j}$ be the nonlinear solutions defined above. Then when $d \geq 4$,

$$
\begin{aligned}
\left\|v_{n}^{j} v_{n}^{k}\right\|_{L_{t, x}^{\frac{2(d+2)}{d-2}}} & +\left\|v_{n}^{j} \nabla v_{n}^{k}\right\|_{L_{t, x}^{\frac{d+2}{d-1}}}+\left\||x| v_{n}^{j} v_{n}^{k}\right\|_{L_{t, x}^{\frac{d+2}{d-1}}} \\
& +\left\|\left(\nabla v_{n}^{j}\right)\left(\nabla v_{n}^{k}\right)\right\|_{L_{t, x}} \frac{2(d+2)}{d x}+\left\||x|^{2} v_{n}^{j} v_{n}^{k}\right\|_{L_{t, x}} \frac{2(d+2)}{d} \rightarrow 0
\end{aligned}
$$

as $n \rightarrow \infty$. When $d=3$, the same statement holds with the last two expressions replaced by

$$
\left\|\left(\nabla v_{n}^{j}\right)\left(\nabla v_{n}^{k}\right)\right\|_{L_{t}^{5} L_{x}^{\frac{15}{11}}}+\left\||x|^{2} v_{n}^{j} v_{n}^{k}\right\|_{L_{t}^{5} L_{x}^{\frac{30}{11}}} \rightarrow 0
$$

Proof. We spell out the details for the $\left\|v_{n}^{j}|x| v_{n}^{k}\right\|_{\frac{d+2}{\frac{d-1}{d-1}}}$ term. Consider first the case $d \geq 4$. As $2<\frac{2(d+2)}{d}<d$, by Proposition 5.1 we can approximate $v_{n}^{j}$ in $\dot{X}^{1}$ by test functions

$$
c_{n}^{j} \tilde{G}_{n} \psi^{j}, \quad \psi^{j} \in C_{c}^{\infty}\left(\mathbf{R} \times \mathbf{R}^{d}\right), \quad c_{n}^{j}(t)=e^{-\frac{i\left(t-t_{n}^{j}\right)\left|x_{n}^{j}\right|^{2}}{2}} .
$$

By Hölder and a change of variables,

$$
\begin{aligned}
& \left\|v_{n}^{j}|x| v_{n}^{k}\right\|_{L_{t, x}^{\frac{d+2}{d-1}}} \leq\left\|\left(v_{n}^{j}-c_{n}^{j} \tilde{G}_{n}^{j} \psi^{j}\right)|x| v_{n}^{k}\right\|_{L_{t, x}^{\frac{d+2}{d-1}}} \\
& +\left\||x| \tilde{G}_{n}^{j} \psi^{j}\left(v_{n}^{k}-c_{n}^{k} \tilde{G}_{n}^{k} \psi^{k}\right)\right\|_{L_{t, x}^{\frac{d+2}{d-1}}}+\left\||x| \tilde{G}_{n}^{j} \psi^{j} \tilde{G}_{n}^{k} \psi^{k}\right\|_{L_{t, x}^{\frac{d+2}{d-1}}} \\
& \leq\left\|\left(v_{n}^{j}-c_{n}^{j} \tilde{G}_{n}^{j} \psi^{j}\right)\right\|_{L_{t, x}^{\frac{2(d+2)}{d-2}}}\left\|v_{n}^{k}\right\|_{\dot{X}^{1}} \\
& +\left\|\psi^{j}\right\|_{L_{t, x} \frac{2(d+2)}{d-2}}\left\|\left(v_{n}^{k}-c_{n}^{k} \tilde{G}_{n}^{k} \psi^{k}\right)\right\|_{\dot{X}^{1}}+\left\|\left(\tilde{G}_{n}^{j} \psi^{j}\right)|x|\left(\tilde{G}_{n}^{k} \psi^{k}\right)\right\|_{L_{t, x}^{\frac{d+2}{d-1}}}
\end{aligned}
$$

By first choosing $\psi^{j}$, then $\psi^{k}$, then invoking the previous lemma, one obtains for any $\varepsilon>0$ that

$$
\limsup _{n \rightarrow \infty}\left\|v_{n}^{j}|x| v_{n}^{k}\right\|_{L_{t, x}^{\frac{d+2}{d-1}}} \leq \varepsilon
$$


When $d=3$, we also approximate $v_{n}^{j}$ in $\dot{X}^{1}$ (which is possible because the exponent $\frac{30}{11}$ in the definition of $\dot{X}^{1}$ is less than 3 ), and estimate

$$
\begin{aligned}
& \left\|v_{n}^{j}|x| v_{n}^{k}\right\|_{L_{t, x}^{\frac{5}{2}}} \\
& \leq\left\|\left(v_{n}^{j}-c_{n}^{j} \tilde{G}_{n}^{j} \psi^{j}\right)|x| v_{n}^{k}\right\|_{L_{t, x}^{\frac{5}{2}}}+\left\||x| \tilde{G}_{n}^{j} \psi^{j}\left(v_{n}^{k}-c_{n}^{k} \tilde{G}_{n}^{k} \psi^{k}\right)\right\|_{L_{t, x}^{\frac{5}{2}}}+\left\||x| \tilde{G}_{n}^{j} \psi^{j} \tilde{G}_{n}^{k} \psi^{k}\right\|_{L_{t, x}^{\frac{5}{2}}} \\
& \leq\left\|\left(v_{n}^{j}-c_{n}^{j} \tilde{G}_{n}^{j} \psi^{j}\right)\right\|_{L_{t, x}^{10}}\left\|v_{n}^{k}\right\|_{\dot{Y}^{1}} \\
& +\left\|\psi^{j}\right\|_{L_{t}^{5} L_{x}^{30}}\left\|v_{n}^{k}-c_{n}^{k} \tilde{G}_{n}^{k} \psi^{k}\right\|_{\dot{X}^{1}}+\left\|\left(\tilde{G}_{n}^{j} \psi^{j}\right)|x|\left(\tilde{G}_{n}^{k} \psi^{k}\right)\right\|_{L_{t, x}^{\frac{5}{2}}}
\end{aligned}
$$

which, just as above, can be made arbitrarily small as $n \rightarrow \infty$. Similar approximation arguments deal with the other terms.

Let us verify Claim (ii) above. In fact we will show that

$$
\limsup _{n \rightarrow \infty}\left\|u_{n}^{J}\right\|_{\dot{X}^{1}\left(\left[-\frac{1}{2}, \frac{1}{2}\right]\right)} \lesssim_{E_{c}, \delta} 1 \text { uniformly in } J \text {. }
$$

First, we have

$$
S\left(u_{n}^{J}\right)=\iint\left|\sum_{j=1}^{J} v_{n}^{j}+e^{-i t H} w_{n}^{J}\right|^{\frac{2(d+2)}{d-2}} d x d t \lesssim S\left(\sum_{j=1}^{J} v_{n}^{j}\right)+S\left(e^{-i t H} w_{n}^{J}\right) .
$$

By the properties of the LPD, $\lim _{J \rightarrow J^{*}} \lim \sup _{n \rightarrow \infty} S\left(e^{-i t H} w_{n}^{J}\right)=0$. Recalling (6.3), we have

$$
\begin{aligned}
& S\left(\sum_{j=1}^{J} v_{n}^{j}\right)=\left\|\left(\sum_{j=1}^{J} v_{n}^{j}\right)^{2}\right\|_{L_{t, x}^{\frac{d+2}{d-2}}}^{\frac{d+2}{d-2}} \leq\left(\sum_{j=1}^{J}\left\|v_{n}^{j}\right\|_{L_{t, x}}^{2} \frac{2(d+2)}{d-2}+\sum_{j \neq k}\left\|v_{n}^{j} v_{n}^{k}\right\|_{L_{t, x}^{\frac{d+2}{d-2}}}\right)^{\frac{d+2}{d-2}} \\
& \lesssim\left(\sum_{j=1}^{J} E\left(\phi_{n}^{j}\right)+o_{J}(1)\right)^{\frac{d+2}{d-2}}
\end{aligned}
$$

where for the last line we invoked Lemma 6.3. Since energy decoupling implies $\limsup \sup _{n \rightarrow \infty} \sum_{j=1}^{J} E\left(\phi_{n}^{j}\right) \leq E_{c}$, we obtain $\lim _{J \rightarrow J^{*}} \lim \sup _{n \rightarrow \infty} S\left(u_{n}^{J}\right) \lesssim_{E_{c}, \delta} 1$.

Mimicking this argument lets us show that

$$
\limsup _{n \rightarrow \infty}\left(\left\|\nabla u_{n}^{J}\right\|_{L_{t, x}^{\frac{2(d+2)}{d}}}+\left\||x| u_{n}^{J}\right\|_{L_{t, x} \frac{2(d+2)}{d}}\right) \lesssim_{E_{c}, \delta} 1 \text { uniformly in } J .
$$

This completes the verification of property (ii) in the case $d \geq 4$. The case $d=3$ is dealt with in a similar fashion.

Remark. The above argument shows that for each $J$ and each $\eta>0$, there exists $J^{\prime} \leq J$ such that

$$
\limsup _{n \rightarrow \infty}\left\|\sum_{j=J^{\prime}}^{J} v_{n}^{j}\right\|_{\dot{X}^{1}\left(\left[-\frac{1}{2}, \frac{1}{2}\right]\right)} \leq \eta .
$$

It remains to check property (iii) above, namely, that

$$
\lim _{J \rightarrow J^{*}} \limsup _{n \rightarrow \infty}\left\|H^{1 / 2} e_{n}^{J}\right\|_{N\left(\left[-\frac{1}{2}, \frac{1}{2}\right]\right)}=0 .
$$

Writing $F(z)=|z|^{\frac{4}{d-2}} z$, we decompose

$$
e_{n}^{J}=\left[\sum_{j=1}^{J} F\left(v_{n}^{j}\right)-F\left(\sum_{j=1}^{J} v_{n}^{j}\right)\right]+\left[F\left(u_{n}^{J}-e^{-i t H} w_{n}^{J}\right)-F\left(u_{n}^{J}\right)\right]=(a)+(b) .
$$


Consider (a) first. Suppose $d \geq 6$. Using the chain rule $\nabla F(u)=F_{z}(u) \nabla u+$ $F_{\bar{z}}(u) \overline{\nabla u}$ and the estimates

$$
\left|F_{z}(z)\right|+\left|F_{\bar{z}}(z)\right|=O\left(|z|^{\frac{4}{d-2}}\right),\left|F_{z}(z)-F_{z}(w)\right|+\left|F_{\bar{z}}(z)-F_{\bar{z}}(w)\right|=O\left(|z-w|^{\frac{4}{d-2}}\right),
$$

we compute

$$
|\nabla(a)| \lesssim \sum_{j=1}^{J} \sum_{k \neq j}\left|v_{n}^{k}\right|^{\frac{4}{d-2}}\left|\nabla v_{n}^{j}\right| .
$$

By Hölder, Lemma 6.3, and the induction hypothesis (6.3),

$$
\|\nabla(a)\|_{L_{t, x}^{\frac{2(d+2)}{d+4}}} \lesssim \sum_{j=1}^{J} \sum_{k \neq j}\left\|\left|v_{n}^{k}\left\|\nabla v_{n}^{j} \mid\right\|_{L_{t, x}}^{\frac{4}{d-2}}\left\|\nabla v_{n}^{k}\right\|_{L_{t, x}^{\frac{d+2}{d-1}}}^{\frac{2-6}{d-2}}=o_{J}(1)\right.\right.
$$

as $n \rightarrow \infty$. When $3 \leq d \leq 5$, we have instead

$$
|\nabla(a)| \lesssim \sum_{j=1}^{J} \sum_{k \neq j}\left|v_{n}^{k}\right|\left|\nabla v_{n}^{j}\right| O\left(\left|\sum_{k=1}^{J} v_{n}^{k}\right|^{\frac{6-d}{d-2}}+\left|v_{n}^{j}\right|^{\frac{6-d}{d-2}}\right),
$$

thus

$$
\|\nabla(a)\|_{L_{t, x}^{\frac{2(d+2)}{d+4}}} \lesssim J\left(\sum_{j=1}^{J}\left\|v_{n}^{j}\right\|_{L_{t, x}^{\frac{2(d-2)}{d-2}}}^{\frac{6-d}{d-2}}\right) \sum_{j=1}^{J} \sum_{k \neq j}\left\|\left|v_{n}^{k}\left\|\nabla v_{n}^{j} \mid\right\|_{L_{t, x}^{\frac{d+2}{d-1}}}=o_{J}(1) .\right.\right.
$$

Similarly, writing

$$
|(a)| \leq \sum_{j=1}^{J}\left|v_{n}^{j}\right|^{\frac{4}{d-2}}-\left.\left|\sum_{k=1}^{J} v_{n}^{k}\right|^{\frac{4}{d-2}}|| v_{n}^{j}\left|\lesssim \sum_{j=1}^{J} \sum_{k \neq j}\right| v_{n}^{j}|| v_{n}^{k}\right|^{\frac{4}{d-2}},
$$

we have

$$
\|x(a)\|{ }_{L_{t, x} \frac{2(d+2)}{d+4}} \sum_{j=1}^{J} \sum_{k \neq j}\left\||x| v_{n}^{j}\right\|_{L_{t, x}^{\frac{d-6}{d-2}}}^{\frac{2(d+2)}{d}}\left\||x| v_{n}^{j} v_{n}^{k}\right\|_{L_{t, x}^{\frac{d+2}{d-1}}}^{\frac{4}{d-2}}=o_{J}(1) .
$$

When $3 \leq d \leq 5$,

$$
|(a)| \lesssim \sum_{j=1}^{J} \sum_{k \neq j}\left|v_{n}^{j}\right| v_{n}^{k} \mid O\left(\left|\sum_{k=1}^{J} v_{n}^{k}\right|^{\frac{6-d}{d-2}}+\left|v_{n}^{j}\right|^{\frac{6-d}{d-2}}\right),
$$

hence also

$$
\||x|(a)\|_{L_{t, x}^{\frac{2(d+2)}{d+4}}}^{\frac{(d+x}{}}=o_{J}(1) .
$$

Summing up,

$$
\left\|H^{1 / 2}(a)\right\|_{L_{t, x} \frac{2(d+2)}{d+4}} \lesssim\|\nabla(a)\|_{L_{t, x}^{\frac{2(d+2)}{d+4}}}+\|x(a)\|_{L_{t, x} \frac{2(d+2)}{d+4}}=o_{J}(1) .
$$


We now estimate (b), restricting temporarily to dimensions $d \geq 4$. When $d \geq 6$, write

$$
\begin{aligned}
(b) & =F\left(u_{n}^{J}-e^{-i t H} w_{n}^{J}\right)-F\left(u_{n}^{J}\right) \\
& =\left(\left|u_{n}^{J}-e^{-i t H} w_{n}^{J}\right|^{\frac{4}{d-2}}-\left|u_{n}^{J}\right|^{\frac{4}{d-2}}\right) \sum_{j=1}^{J} v_{n}^{j}-\left(e^{-i t H} w_{n}^{J}\right)\left|u_{n}^{J}\right|^{\frac{4}{d-2}} \\
& =O\left(\left|e^{-i t H} w_{n}^{J}\right|^{\frac{4}{d-2}}\right) \sum_{j=1}^{J} v_{n}^{j}-\left(e^{-i t H} w_{n}^{J}\right)\left|u_{n}^{J}\right|^{\frac{4}{d-2}},
\end{aligned}
$$

and apply Hölder's inequality:

$$
\begin{aligned}
\||x|(b)\|_{L_{t, x}^{\frac{2(d+2)}{d+4}}} & \lesssim\left\|e^{-i t H} w_{n}^{J}\right\|_{L_{t, x}^{\frac{2(d+2)}{d-2}}}^{\frac{4}{d-2}}\left\|\sum_{j=1}^{J}|x| v_{n}^{j}\right\|_{L_{t, x}} \frac{2(d+2)}{d} \\
& +\left\||x| u_{n}^{J}\right\|_{L_{t, x}^{\frac{4}{d-2}}}^{\frac{2(d+2)}{d}}\left\||x| e^{-i t H} w_{n}^{J}\right\|_{L_{t, x}^{\frac{2-6}{d-2}}}^{\frac{2(d+2)}{d}}\left\|e^{-i t H} w_{n}^{J}\right\|_{L_{t, x}^{\frac{2(d+2)}{d-2}}}^{\frac{2}{d-2}}
\end{aligned}
$$

When $d=4,5$,

$$
(b)=\left(e^{-i t H} w_{n}^{J}\right) O\left(\left|u_{n}^{J}\right|^{\frac{6-d}{d-2}}+\left|u_{n}^{J}-e^{-i t H} w_{n}^{J}\right|^{\frac{6-d}{d-2}}\right) \sum_{j=1}^{J} v_{n}^{j}-\left(e^{-i t H} w_{n}^{J}\right)\left|u_{n}^{J}\right|^{\frac{4}{d-2}},
$$

thus

$$
\begin{aligned}
& \left.\||x|(b)\|\right|_{L_{t, x} \frac{2(d+2)}{d+4}} \\
& \lesssim\left\|e^{-i t H} w_{n}^{J}\right\|_{L_{t, x}^{\frac{2(d+2)}{d-2}}}\left\||x| \sum_{j=1}^{J} v_{n}^{j}\right\|_{L_{t, x}^{\frac{2(d+2)}{d}}}\left(\left\|u_{n}^{J}\right\|_{L_{t, x}^{\frac{2(d-2}{d-2}}}^{\frac{\frac{6-d}{d-2}}{L^{2}}}+\left\|e^{-i t H} w_{n}^{J}\right\|_{L_{t, x}^{\frac{2(d+2)}{d-2}}}^{\frac{6-d}{d-2}}\right) \\
& +\left\|e^{-i t H} w_{n}^{J}\right\|_{L_{t, x}^{\frac{2(d+2)}{d-2}}}\left\|x u_{n}^{J}\right\|_{L_{t, x}^{\frac{2(d+2)}{d}}}\left\|u_{n}^{J}\right\|_{L_{t, x}^{\frac{2-d}{d-2}}}^{\frac{2(d+2)}{d-2}} .
\end{aligned}
$$

Using (6.5), Strichartz, and the decay property (6.1), we get

$$
\lim _{J \rightarrow J *} \limsup _{n \rightarrow \infty}\||x|(b)\|_{L_{t, x} \frac{2(d+2)}{d+4}}=0 .
$$

It remains to bound $\nabla(b)$. By the chain rule,

$$
\begin{aligned}
\nabla(b) & \left.\lesssim\left|e^{-i t H} w_{n}^{J}\right|^{\frac{4}{d-2}}|| \sum_{j=1}^{J} \nabla v_{n}^{j}|+| u_{n}^{J}\right|^{\frac{4}{d-2}}\left|\nabla e^{-i t H} w_{n}^{J}\right| \\
& =\left(b^{\prime}\right)+\left(b^{\prime \prime}\right) .
\end{aligned}
$$

The first term $\left(b^{\prime}\right)$ can be handled in the manner of (6.7) above. We now concern ourselves with (b"). Fix a small parameter $\eta>0$, and use the above remark to obtain $J^{\prime}=J^{\prime}(\eta) \leq J$ such that

$$
\left\|\sum_{j=J^{\prime}}^{J} v_{n}^{j}\right\|_{\dot{X}^{1}} \leq \eta
$$


By the subadditivity of $z \mapsto|z|^{\frac{4}{d-2}}$ (which is true up to a constant when $d=4,5$ ) and Hölder,

$$
\begin{aligned}
& \left\|\left(b^{\prime \prime}\right)\right\|_{\frac{2(d+2)}{d+4}}=\left\|\left|\sum_{j=1}^{J} v_{n}^{j}+e^{-i t H} w_{n}^{J}\right|^{\frac{4}{d-2}}\left|\nabla e^{-i t H} w_{n}^{J}\right|\right\|_{L_{t, x} \frac{2(d+2)}{d+4}} \\
& \lesssim\left\|e^{-i t H} w_{n}^{J}\right\|_{L_{t, x}^{\frac{2(d+2}{d-2}}}^{\frac{4}{d-2}}\left\|H^{1 / 2} e^{-i t H} w_{n}^{J}\right\|_{L_{t, x}}^{\frac{2(d+2)}{d}}+\left\|\sum_{j=J^{\prime}}^{J} v_{n}^{j}\right\|_{L_{t, x}^{\frac{2(d+2}{d-2}}}^{\frac{4}{d-2}}\left\|H^{1 / 2} e^{-i t H} w_{n}^{J}\right\|_{L_{t, x} \frac{2(d+2)}{d}} \\
& +C_{J^{\prime}} \sum_{j=1}^{J^{\prime}-1}\left\|\nabla e^{-i t H} w_{n}^{J}\right\|_{L_{t, x}^{\frac{d-6}{d-2}}}^{\frac{2(d+2)}{d,}}\left\|\mid v_{n}^{j}\right\| \nabla e^{-i t H} w_{n}^{J} \|_{L_{t, x}^{\frac{d+2}{d-1}}}^{\frac{4}{d-2}} .
\end{aligned}
$$

By Strichartz and the decay of $e^{-i t H} w_{n}^{J}$ in $L_{t, x}^{\frac{2(d+2)}{d-2}}$, the first term goes to 0 as $J \rightarrow \infty, n \rightarrow \infty$. By Strichartz and the definition of $J^{\prime}$, the second term is bounded by

$$
\eta^{\frac{4}{d-2}}\left\|w_{n}^{J}\right\|_{\Sigma}
$$

which can be made arbitrarily small since $\limsup _{n \rightarrow \infty}\left\|w_{n}^{J}\right\|_{\Sigma}$ is bounded uniformly in $J$. To finish, we show that for each fixed $j$

$$
\lim _{J \rightarrow J^{*}} \limsup _{n \rightarrow \infty}\left\|\left|v_{n}^{j}\right| \nabla e^{-i t H} w_{n}^{J}\right\|_{L_{t, x}^{\frac{d+2}{d-1}}}=0 .
$$

For any $\varepsilon>0$, there exist $\psi^{j} \in C_{c}^{\infty}\left(\mathbf{R} \times \mathbf{R}^{d}\right)$ such that if

$$
c_{n}^{j}=e^{-\frac{i\left(t-t_{n}^{j}\right)\left|x_{n}^{j}\right|^{2}}{2}}
$$

then

$$
\limsup _{n \rightarrow \infty}\left\|v_{n}^{j}-c_{n}^{j} \tilde{G}_{n}^{j} \psi^{j}\right\|_{L_{t, x}^{\frac{2(d+2)}{d-2}}\left(\left[-\frac{1}{2}, \frac{1}{2}\right]\right)}<\varepsilon
$$

Note that $\tilde{G}_{n}^{j} \psi^{j}$ is supported on the set

$$
\left\{\left|t-t_{n}^{j}\right| \lesssim\left(N_{n}^{j}\right)^{-2},\left|x-x_{n}^{j}\right| \lesssim\left(N_{n}^{j}\right)^{-1}\right\} .
$$

Thus for all $n$ sufficiently large,

$$
\begin{aligned}
& \left\|v_{n}^{j} \nabla e^{-i t H} w_{n}^{J}\right\|_{L_{t, x}^{\frac{d+2}{d-1}}} \\
& \leq\left\|v_{n}^{j}-c_{n}^{j} \tilde{G}_{n}^{j} \psi^{j}\right\|_{L_{t, x}^{\frac{2(d+2)}{d-2}}}\left\|\nabla e^{-i t H} w_{n}^{J}\right\|_{L_{t, x}} \frac{2(d+2)}{d}+\left\|\tilde{G}_{n}^{j} \psi^{j} \nabla e^{-i t H} w_{n}^{J}\right\|_{L_{t, x}^{\frac{d+2}{d-1}}} \\
& \lesssim_{E_{c}} \varepsilon+\left\|\left(\tilde{G}_{n}^{j} \psi^{j}\right) \nabla e^{-i t H} w_{n}^{J}\right\|_{L_{t, x}^{\frac{d+2}{d-1}}} \cdot
\end{aligned}
$$

By Hölder, noting that $\frac{d+2}{d-1} \leq 2$ whenever $d \geq 4$,

$$
\begin{aligned}
\left\|\left(\tilde{G}_{n}^{j} \psi^{j}\right) \nabla e^{-i t H} w_{n}^{J}\right\|_{L_{t, x}^{\frac{d+2}{d-1}}} & \lesssim \varepsilon\left(N_{n}^{j}\right)^{\frac{d-2}{2}}\left\|\nabla e^{-i t H} w_{n}^{J}\right\|_{L_{t, x}^{\frac{d+2}{d-1}}\left(\left|t-t_{n}^{j}\right| \lesssim\left(N_{n}^{j}\right)^{-2},\left|x-x_{n}^{j}\right| \lesssim\left(N_{n}^{j}\right)^{-1}\right)} \\
& \lesssim N_{n}^{j}\left\|\nabla e^{-i t H} w_{n}^{J}\right\|_{L_{t, x}^{2}}\left(\left|t-t_{n}^{j}\right| \lesssim\left(N_{n}^{j}\right)^{-2},\left|x-x_{n}^{j}\right| \lesssim\left(N_{n}^{j}\right)^{-1}\right)
\end{aligned}
$$

Since $\left(N_{n}^{j}\right)^{-2}\left|x_{n}^{j}\right|=O\left(\left(N_{n}^{j}\right)^{-1}\right)$, Corollary 2.9 implies

$$
\left\|v_{n}^{j} \nabla e^{-i t H} w_{n}^{J}\right\|_{L_{t, x}^{\frac{d+2}{d-1}}} \lesssim \varepsilon+C_{\varepsilon, E_{c}}\left\|e^{-i t H} w_{n}^{J}\right\|_{L_{t, x}^{\frac{1}{d}}}^{\frac{2(d+2)}{d-2}} .
$$


Sending $n \rightarrow \infty$, then $J \rightarrow J^{*}$, then $\varepsilon \rightarrow 0$ establishes (6.8), and with it, Property (iii).

When $d=3$, we estimate $(b)$ instead with the $L_{t}^{\frac{5}{3}} L_{x}^{\frac{30}{23}}$ dual Strichartz norm. Write

$$
(b)=\left(e^{-i t H} w_{n}^{J}\right) v_{n}^{j} O\left(\left|u_{n}^{J}\right|^{3}+\left|u_{n}^{J}-e^{-i t H} w_{n}^{J}\right|^{3}\right) \sum_{j=1}^{J} v_{n}^{j}-\left(e^{-i t H} w_{n}^{J}\right)\left|u_{n}^{J}\right|^{4},
$$

and apply Hölder's inequality:

$$
\begin{aligned}
\||x|(b)\|_{L_{t}^{\frac{5}{3}} L_{x}^{\frac{30}{2}}} & \lesssim\left\|e^{-i t H} w_{n}^{J}\right\|_{L_{t, x}^{10}}\left\|u_{n}^{J}\right\|_{L_{t, x}^{10}}^{3}\left\|H^{1 / 2} u_{n}^{J}\right\|_{L_{t}^{5} L_{x}^{\frac{30}{11}}} \\
& +\left\|e^{-i t H} w_{n}^{J}\right\|_{L_{t, x}^{10}}\left(\left\|u_{n}^{J}\right\|_{L_{t, x}^{10}}^{3}+\left\|e^{-i t H} w_{n}^{J}\right\|_{L_{t, x}^{10}}^{3}\right)\left\|H^{\frac{1}{2}} \sum_{j=1}^{J} v_{n}^{j}\right\|_{L_{t}^{5} L_{x}^{\frac{30}{11}}} .
\end{aligned}
$$

Using (6.1) and (6.5), we have

$$
\lim _{J \rightarrow J *} \limsup _{n \rightarrow \infty}\||x|(b)\|_{L_{t}^{\frac{5}{3}} L_{x}^{\frac{30}{11}}=0 .}
$$

It remains to bound $\nabla(b)$. By the chain rule,

$$
\begin{aligned}
\nabla(b) & =O\left(\left(\left|u_{n}^{J}-e^{-i t H} w_{n}^{J}\right|^{4}-\left|u_{n}^{J}\right|^{4}\right) \nabla \sum_{j=1}^{J} v_{n}^{j}\right)+\left|u_{n}^{J}\right|^{4}\left|\nabla e^{-i t H} w_{n}^{J}\right| \\
& =\left(b^{\prime}\right)+\left(b^{\prime \prime}\right) .
\end{aligned}
$$

The first term $\left(b^{\prime}\right)$ can be treated in the manner of $\||x|(b)\|_{L_{t}^{\frac{5}{3}} L_{x}^{\frac{30}{23}}}$ above. We now concern ourselves with $\left(b^{\prime \prime}\right)$. Fix a small parameter $\eta>0$, and use the above remark to obtain $J^{\prime}=J^{\prime}(\eta) \leq J$ such that

$$
\left\|\sum_{j=J^{\prime}}^{J} v_{n}^{j}\right\|_{\dot{X}^{1}} \leq \eta
$$

Thus by the triangle inequality and Hölder,

$$
\begin{aligned}
\left\|\left(b^{\prime \prime}\right)\right\|_{L_{t}^{\frac{5}{3}} L_{x}^{\frac{30}{23}}} & =\left\|\left|\sum_{j=1}^{J} v_{n}^{j}+e^{-i t H} w_{n}^{J}\right|^{4}\left(e^{-i t H} w_{n}^{J}\right)\right\|_{L_{t}^{\frac{5}{3}} L_{x}^{\frac{30}{23}}} \\
& \lesssim\left\|e^{-i t H} w_{n}^{J}\right\|_{L_{t, x}^{10}}^{4}\left\|H^{\frac{1}{2}} e^{-i t H} w_{n}^{J}\right\|_{L_{t}^{5} L_{x}^{\frac{30}{11}}} \\
& +\left\|\left|\sum_{j=J^{\prime}}^{J} v_{n}^{j}\right|^{4}\left|\nabla e^{-i t H} w_{n}^{J}\right|\right\|_{L_{t}^{\frac{5}{3}} L_{x}^{\frac{30}{23}}}+C_{J^{\prime}} \sum_{j=1}^{J^{\prime}}\left\|\left|v_{n}^{j}\right|^{4} \nabla e^{-i t H} w_{n}^{J}\right\|_{L_{t}^{\frac{5}{3}} L_{x}^{\frac{30}{23}}} \\
& \lesssim\left\|e^{-i t H} w_{n}^{J}\right\|_{L_{t, x}^{10}}^{4}\left\|H^{\frac{1}{2}} e^{-i t H} w_{n}^{J}\right\|_{L_{t}^{5} L_{x}^{\frac{30}{11}}} \\
& +\left\|\sum_{j=J^{\prime}}^{J} v_{n}^{j}\right\|_{\dot{X}^{1}}^{4}\left\|\left|\nabla e^{-i t H} w_{n}^{J}\right|\right\|_{L_{t}^{5} L_{x}^{\frac{30}{11}}}+C_{J^{\prime}} \sum_{j=1}^{J^{\prime}}\left\|\left|v_{n}^{j}\right|^{4} \nabla e^{-i t H} w_{n}^{J}\right\|_{L_{t}^{\frac{5}{3}} L_{x}^{\frac{30}{23}}}
\end{aligned}
$$

By Strichartz and the decay of $e^{-i t H} w_{n}^{J}$ in $L_{t, x}^{10}$, the first term goes to 0 as $J \rightarrow$ $\infty, n \rightarrow \infty$. By Strichartz and the definition of $J^{\prime}$, the second term is bounded by

$$
\eta^{4}\left\|w_{n}^{J}\right\|_{\Sigma}
$$


which can be made arbitrarily small since $\lim \sup _{n \rightarrow \infty}\left\|w_{n}^{J}\right\|_{\Sigma}$ is bounded uniformly in $J$. To finish, we show that for each fixed $j$

$$
\lim _{J \rightarrow J^{*}} \limsup _{n \rightarrow \infty}\left\|\left|v_{n}^{j}\right|^{4} \nabla e^{-i t H} w_{n}^{J}\right\|_{L_{t}^{\frac{5}{3}} L_{x}^{\frac{30}{10}}}=0 .
$$

By Hölder,

$$
\left\|\left|v_{n}^{j}\right|^{4} \nabla e^{-i t H} w_{n}^{J}\right\|_{L_{t}^{\frac{5}{3}} L_{x}^{\frac{30}{23}}} \leq\left\|v_{n}^{j}\right\|_{L_{t, x}^{10}}^{3}\left\|v_{n}^{j} \nabla e^{-i t H} w_{n}^{J}\right\|_{L_{t}^{\frac{10}{3}} L_{x^{\frac{15}{7}}}}
$$

so by (6.3) it suffices to show

$$
\lim _{J \rightarrow J^{*}} \limsup _{n \rightarrow \infty}\left\|v_{n}^{j} \nabla e^{-i t H} w_{n}^{J}\right\|_{L_{t}^{\frac{10}{3}}} L_{x}^{\frac{15}{j}}=0 .
$$

For any $\varepsilon>0$, there exists $\psi^{j} \in C_{c}^{\infty}\left(\mathbf{R} \times \mathbf{R}^{3}\right)$ and functions $c_{n}^{j}(t),\left|c_{n}^{j}\right| \equiv 1$ such that

$$
\limsup _{n \rightarrow \infty}\left\|v_{n}^{j}-c_{n}^{j} \tilde{G}_{n}^{j} \psi^{j}\right\|_{L_{t, x}^{10}\left(\left[-\frac{1}{2}, \frac{1}{2}\right]\right)}<\varepsilon
$$

Note that $\tilde{G}_{n}^{j} \psi^{j}$ is supported on the set

$$
\left\{\left|t-t_{n}^{j}\right| \lesssim\left(N_{n}^{j}\right)^{-2},\left|x-x_{n}^{j}\right| \lesssim\left(N_{n}^{j}\right)^{-1}\right\} .
$$

Thus for all $n$ sufficiently large,

$$
\begin{aligned}
& \left\|v_{n}^{j} \nabla e^{-i t H} w_{n}^{J}\right\|_{L_{t}^{\frac{10}{3}}} L_{x}^{\frac{15}{7}} \\
& \leq\left\|v_{n}^{j}-c_{n}^{j} \tilde{G}_{n}^{j} \psi^{j}\right\|_{L_{t, x}^{10}}\left\|\nabla e^{-i t H} w_{n}^{J}\right\|_{L_{t}^{5} L_{x}^{\frac{30}{11}}}+\left\|\tilde{G}_{n}^{j} \psi^{j} \nabla e^{-i t H} w_{n}^{J}\right\|_{L_{t}^{\frac{10}{3}}} L_{x}^{\frac{15}{7}} \\
& \lesssim_{E_{c}} \varepsilon+\left\|\left(\tilde{G}_{n}^{j} \psi^{j}\right) \nabla e^{-i t H} w_{n}^{J}\right\|_{L_{t}^{\frac{10}{3}}} \frac{15}{L_{x}^{7}}
\end{aligned}
$$

From the definition of the operators $\tilde{G}_{n}^{j}$, we have

$\left\|\left(\tilde{G}_{n}^{j} \psi^{j}\right) \nabla e^{-i t H} w_{n}^{J}\right\|_{L_{t}^{\frac{10}{3}} L_{x}^{\frac{15}{7}} \lesssim \varepsilon} N_{n}^{\frac{1}{2}}\left\|\nabla e^{-i t H} w_{n}^{J}\right\|_{L_{t}^{\frac{10}{3}} L_{x}^{\frac{15}{7}}\left(\left|t-t_{n}^{j}\right| \lesssim\left(N_{n}^{j}\right)^{-2},\left|x-x_{n}^{j}\right| \lesssim\left(N_{n}^{j}\right)^{-1}\right)}$.

Since $\left(N_{n}^{j}\right)^{-2}\left|x_{n}^{j}\right|=O\left(\left(N_{n}^{j}\right)^{-1}\right)$, Corollary 2.9 implies

$$
\left\|v_{n}^{j} \nabla e^{-i t H} w_{n}^{J}\right\|_{L_{t}^{\frac{10}{3}} L_{x}^{\frac{15}{7}}} \lesssim \varepsilon+C_{\varepsilon}\left\|e^{-i t H} w_{n}^{J}\right\|_{L_{t, x}^{10}}^{\frac{1}{9}}\left\|w_{n}^{J}\right\|_{\Sigma}^{\frac{8}{9}} .
$$

Sending $n \rightarrow \infty$, then $J \rightarrow J^{*}$, then $\varepsilon \rightarrow 0$ establishes (6.10), and with it, Property (iii). This completes the treatment of the case $d=3$.

By perturbation theory, $\lim \sup _{n \rightarrow \infty} S_{(-T, T)} \leq C\left(E_{c}\right)<\infty$, contrary to the Palais-Smale hypothesis. This rules out Case 2 and completes the proof of Proposition 6.1.

Armed with Proposition 6.1, we can finish the proof of Theorem 1.2

Proof of Theorem 1.2. Suppose the theorem failed. In the defocusing case, there exist $E_{c} \in(0, \infty)$ and a sequence of solutions $u_{n}$ with $E\left(u_{n}\right) \rightarrow E_{c}$ and $S_{\left(-\frac{1}{n}, 0\right]}\left(u_{n}\right) \rightarrow$ $\infty$ and $S_{\left[0, \frac{1}{n}\right)}\left(u_{n}\right) \rightarrow \infty$. The same is true in the focusing case except $E_{c}$ is restricted to the interval $\left(0, E_{\Delta}(W)\right)$ and $\lim \sup _{n}\left\|u_{n}(0)\right\|_{\dot{H}^{1}}<\|W\|_{\dot{H}^{1}}$. By Proposition 6.1, after passing to a subsequence $u_{n}(0)$ converges in $\Sigma$ to some $\phi$. Let $u_{\infty}$ be the maximal solution to (1.1) with $u_{\infty}(0)=\phi$. By the stability theory and dominated convergence, $\lim _{n \rightarrow \infty} S_{\left[-\frac{1}{n}, \frac{1}{n}\right]}\left(u_{n}\right)=\lim _{n \rightarrow \infty} S_{\left[-\frac{1}{n}, \frac{1}{n}\right]}\left(u_{\infty}\right)=0$, which is a contradiction. 


\section{Proof of Theorem 1.3}

We begin by recalling some facts about the ground state

$$
W(x)=\left(1+\frac{|x|^{2}}{d(d-2)}\right)^{-\frac{d-2}{2}} \in \dot{H}^{1}\left(\mathbf{R}^{d}\right)
$$

This function satisfies the elliptic PDE

$$
\frac{1}{2} \Delta W+W^{\frac{4}{d-2}} W=0 .
$$

It is well-known (c.f. Aubin [1] and Talenti [27]) that the functions witnessing the sharp constant in the Sobolev inequality

$$
\|f\|_{L^{\frac{2 d}{d-2}}\left(\mathbf{R}^{d}\right)} \leq C_{d}\|\nabla f\|_{L^{2}\left(\mathbf{R}^{d}\right)},
$$

are precisely those of the form $f(x)=\alpha W\left(\beta\left(x-x_{0}\right)\right), \alpha \in \mathbf{C}, \beta>0, x_{0} \in \mathbf{R}^{d}$.

For the reader's convenience, we reiterate the definitions of the energy associated to the focusing energy-critical NLS with and without potential:

$$
\begin{aligned}
E_{\Delta}(u) & =\int_{\mathbf{R}^{d}} \frac{1}{2}|\nabla u|^{2}-\left(1-\frac{2}{d}\right)|u|^{\frac{2 d}{d-2}} d x, \\
E(u) & =E_{\Delta}(u)+\frac{1}{2}\|x u\|_{L^{2}}^{2} .
\end{aligned}
$$

Lemma 7.1 (Energy trapping [16]). Suppose $E_{\Delta}(u) \leq\left(1-\delta_{0}\right) E_{\Delta}(W)$.

- If $\|\nabla u\|_{L^{2}} \leq\|\nabla W\|_{L^{2}}$, then there exists $\delta_{1}>0$ depending on $\delta_{0}$ such that

$$
\|\nabla u\|_{L^{2}} \leq\left(1-\delta_{1}\right)\|W\|_{L^{2}},
$$

and $E_{\Delta}(u) \geq 0$.

- If $\|\nabla u\|_{L^{2}} \geq\|\nabla W\|_{L^{2}}$ then there exists $\delta_{2}>0$ depending on $\delta_{0}$ such that

$$
\|\nabla u\|_{L^{2}} \geq\left(1+\delta_{2}\right)\|\nabla W\|_{L^{2}},
$$

and $\frac{1}{2}\|\nabla u\|_{L^{2}}^{2}-\|u\|_{L^{\frac{2 d}{d-2}}}^{\frac{2 d}{d-2}} \leq-\delta_{0} E_{\Delta}(W)$.

Now suppose $E(u)<E_{\Delta}(W)$ and $\|\nabla u\|_{L^{2}} \leq\|\nabla W\|_{L^{2}}$. The energy inequality can be written as

$$
\|u\|_{\Sigma}^{2}+\left(1-\frac{2}{d}\right)\left(\|W\|_{L^{\frac{2 d}{d-2}}}^{\frac{2 d}{d-2}}-\|u\|_{L^{\frac{2 d}{d-2}}}^{\frac{2 d}{d-2}}\right) \leq\|\nabla W\|_{L^{2}}^{2} .
$$

By the variational characterization of $W$, the difference of norms on the left side is nonnegative; therefore

$$
\|u\|_{\Sigma} \leq\|\nabla W\|_{L^{2}}
$$

Combining the above with conservation of energy and a continuity argument, we obtain

Corollary 7.2. Suppose $u: I \times \mathbf{R}^{d} \rightarrow \mathbf{C}$ is a solution to the focusing equation (1.1) with $E(u) \leq\left(1-\delta_{0}\right) E_{\Delta}(W)$. Then there exist $\delta_{1}, \delta_{2}>0$, depending on $\delta_{0}$, such that

- If $\|u(0)\|_{\dot{H}^{1}} \leq\|W\|_{\dot{H}^{1}}$, then

$$
\sup _{t \in I}\|u(t)\|_{\Sigma} \leq\left(1-\delta_{1}\right)\|W\|_{\dot{H}^{1}} \quad \text { and } \quad E(u) \geq 0 .
$$

- If $\|u(0)\|_{\dot{H}^{1}} \geq\|W\|_{\dot{H}^{1}}$, then

$$
\inf _{t \in I}\|u(t)\|_{\Sigma} \geq\left(1+\delta_{2}\right)\|W\|_{\dot{H}^{1}} \quad \text { and } \quad \frac{1}{2}\|\nabla u\|_{2}^{2}-\|u\|_{L^{\frac{2 d}{d-2}}}^{\frac{2 d}{d-2}} \leq-\delta_{0} E_{\Delta}(W) \text {. }
$$


Proof of Theorem 1.3. Let $u$ be the maximal solution to (1.1) with

$$
u(0)=u_{0}, \quad E\left(u_{0}\right)<E_{\Delta}(W), \quad\left\|\nabla u_{0}\right\|_{2} \geq\|\nabla W\|_{2} .
$$

Let $f(t)=\int_{\mathbf{R}^{d}}|x|^{2}|u(t, x)|^{2} d x$. It can be shown 8 , that $f$ is $C^{2}$ on the interval of existence and

$$
f^{\prime \prime}(t)=\int|\nabla u(t, x)|^{2}-2|u(t, x)|^{\frac{2 d}{d-2}}-\frac{1}{2}|x|^{2}|u(t, x)|^{2} d x .
$$

By the corollary, $f^{\prime \prime}$ is bounded above by some fixed $C<0$. Therefore

$$
f(t) \leq A+B t+\frac{C}{2} t^{2}
$$

for some constants $A$ and $B$. It follows that $u$ has a finite lifespan in both time directions.

\section{Bounded LINEAR POTENTIALS}

In this section we show, using a perturbative argument, that

$$
i \partial_{t} u=\left(-\frac{1}{2} \Delta+V\right) u+|u|^{\frac{4}{d-2}} u, \quad u(0)=u_{0} \in H^{1}\left(\mathbf{R}^{d}\right)
$$

is globally wellposed whenever $V$ is a real-valued function with

$$
V_{\text {max }}:=\|V\|_{L^{\infty}}+\|\nabla V\|_{L^{\infty}}<\infty .
$$

This equation defines the Hamiltonian flow of the energy functional

$$
E(u(t))=\int_{\mathbf{R}^{d}} \frac{1}{2}|\nabla u(t, x)|^{2}+V|u(t, x)|^{2}+\frac{d-2}{d}|u|^{\frac{2 d}{d-2}} d x=E(u(0)) .
$$

Solutions to (8.1) also conserve mass:

$$
M(u(t))=\int_{\mathbf{R}^{d}}|u(t, x)|^{2} d x=M(u(0)) .
$$

It will be convenient to assume $V$ is positive and bounded away from 0 . This hypothesis allows us to bound the $H^{1}$ norm of $u$ purely in terms of $E$ instead of both $E$ and $M$, and causes no loss of generality because for sign-indefinite $V$ we could simply consider the conserved quantity $E+C M$ in place of $E$, where $C$ is some positive constant.

Theorem 8.1. For any $u_{0} \in H^{1}\left(\mathbf{R}^{d}\right)$, 8.1) has a unique global solution $u \in$ $C_{t, l o c}^{0} H_{x}^{1}\left(\mathbf{R} \times \mathbf{R}^{d}\right)$. Further, $u$ obeys the spacetime bounds

$$
S_{I}(u) \leq C\left(\left\|u_{0}\right\|_{H^{1}},|I|\right)
$$

for any compact interval $I \subset \mathbf{R}$.

As alluded to at the beginning of this section, the proof uses the strategy pioneered by [29] and treats the term $V u$ as a perturbation to (1.6), which is globally wellposed. Thus Duhamel's formula reads

$$
u(t)=e^{\frac{i t \Delta}{2}} u\left(t_{0}\right)-i \int_{0}^{t} e^{\frac{i(t-s) \Delta}{2}}\left[|u(s)|^{\frac{4}{d-2}} u(s)+V u(s)\right] d s .
$$

We record mostly without proof some standard results in the local theory of (8.1). Introduce the notation




Lemma 8.1 (Local wellposedness). Fix $u_{0} \in H^{1}\left(\mathbf{R}^{d}\right)$, and suppose $T_{0}>0$ is such that

$$
\left\|e^{\frac{i t \Delta}{2}} u_{0}\right\|_{X\left(\left[-T_{0}, T_{0}\right]\right)} \leq \eta \leq \eta_{0}
$$

where $\eta_{0}=\eta_{0}(d)$ is a fixed parameter. Then there exists a positive

$$
T_{1}=T_{1}\left(\left\|u_{0}\right\|_{H^{1}}, \eta, V_{\max }\right)
$$

such that (8.1) has a unique (strong) solution $u \in C_{t}^{0} H_{x}^{1}\left(\left[-T_{1}, T_{1}\right] \times \mathbf{R}^{d}\right)$. Further, if $\left(-T_{\min }, T_{\max }\right)$ is the maximal lifespan of $u$, then $\|\nabla u\|_{S(I)}<\infty$ for every compact interval $I \subset\left(-T_{\min }, T_{\max }\right)$, where $\|\cdot\|_{S(I)}$ is the Strichartz norm defined in Section 2.1.

Proof sketch. Run the usual contraction mapping argument using the Strichartz estimates to show that

$$
\mathcal{I}(u)(t)=e^{\frac{i t \Delta}{2}} u_{0}-i \int_{0}^{t} e^{\frac{i(t-s) \Delta}{2}}\left[|u(s)|^{\frac{4}{d-2}} u(s)+V u(s)\right] d x
$$

has a fixed point in a suitable function space. Estimate the terms involving $V$ in the $L_{t}^{1} L_{x}^{2}$ dual Strichartz norm and choose the parameter $T_{1}$ to make those terms sufficiently small after using Hölder in time.

Lemma 8.2 (Blowup criterion). Let $u:\left(T_{0}, T_{1}\right) \times \mathbf{R}^{d} \rightarrow \mathbf{C}$ be a solution to (8.1) with

$$
\|u\|_{X\left(\left(T_{0}, T_{1}\right)\right)}<\infty
$$

If $T_{0}>-\infty$ or $T_{1}<\infty$, then $u$ can be extended to a solution on a larger time interval. (1.6).

The key result we will rely on is the stability theory for the energy-critical NLS

Lemma 8.3 (Stability [28]). Let $\tilde{u}: I \times \mathbf{R}^{d} \rightarrow \mathbf{C}$ be an approximate solution to equation (1.6) in the sense that

$$
i \partial_{t} \tilde{u}=-\frac{1}{2} \Delta u \pm|\tilde{u}|^{\frac{4}{d-2}} \tilde{u}+e
$$

for some function e. Assume that

$$
\|\tilde{u}\|_{L_{t, x}^{\frac{2(d+2)}{d-2}}} \leq L, \quad\|\nabla u\|_{L_{t}^{\infty} L_{x}^{2}} \leq E,
$$

and that for some $0<\varepsilon<\varepsilon_{0}(E, L)$ one has

$$
\left\|\tilde{u}\left(t_{0}\right)-u_{0}\right\|_{\dot{H}^{1}}+\|\nabla e\|_{N(I)} \leq \varepsilon
$$

where $\|\cdot\|_{N(I)}$ was defined in Section [2.1. Then there exists a unique solution $u: I \times \mathbf{R}^{d} \rightarrow \mathbf{C}$ to (1.6) with $u\left(t_{0}\right)=u_{0}$ which further satisfies the estimates

$$
\|\tilde{u}-u\|_{L_{t, x}^{\frac{2(d+2)}{d-2}}}+\|\nabla(\tilde{u}-u)\|_{S(I)} \lesssim C(E, L) \varepsilon^{c}
$$

where $0<c=c(d)<1$ and $C(E, L)$ is a function which is nondecreasing in each variable.

Proof of Theorem 8.1. It suffices to show that for $T$ sufficiently small depending only on $E=E\left(u_{0}\right)$, the solution $u$ to (8.1) on $[0, T]$ satisfies an a priori estimate

$$
\|u\|_{X([0, T])} \leq C(E) \text {. }
$$


From Lemma 8.2 and energy conservation, it will follow that $u$ is a global solution with the desired spacetime bound.

By Theorem 1.1, the equation

$$
\left(i \partial_{t}+\frac{1}{2} \Delta\right) w=|w|^{\frac{4}{d-2}} w, \quad w(0)=u(0) .
$$

has a unique global solution $w \in C_{t, l o c}^{0} \dot{H}_{x}^{1}\left(\mathbf{R} \times \mathbf{R}^{d}\right)$ with the spacetime bound (1.7). Fix a small parameter $\eta>0$ to be determined shortly, and partition $[0, \infty)$ into $J(E, \eta)$ intervals $I_{j}=\left[t_{j}, t_{j+1}\right)$ so that

$$
\|w\|_{X\left(I_{j}\right)} \leq \eta
$$

For some $J^{\prime}<J$, we then have

$$
[0, T]=\bigcup_{j=0}^{J^{\prime}-1}\left([0, T] \cap I_{j}\right) .
$$

We make two preliminary estimates. By Hölder in time,

$$
\|V u\|_{N\left(I_{j}\right)}+\|\nabla(V u)\|_{N\left(I_{j}\right)} \lesssim C_{V} T\|u\|_{L_{t}^{\infty} H_{x}^{1}\left(I_{j}\right)} \leq \varepsilon
$$

for any $\varepsilon$ provided that $T=T(E, V, \varepsilon)$ is sufficiently small. Further, observe that

$$
\left\|e^{\frac{i\left(t-t_{j}\right) \Delta}{2}} w\left(t_{j}\right)\right\|_{X\left(I_{j}\right)} \leq 2 \eta
$$

for $\eta$ sufficiently small depending only on $d$. Indeed, from the Duhamel formula

$$
w(t)=e^{\frac{i\left(t-t_{j}\right) \Delta}{2}} w\left(t_{j}\right)-i \int_{t_{j}}^{t} e^{\frac{i(t-s) \Delta}{2}}\left(|w|^{\frac{4}{d-2}} w\right)(s) d s,
$$

Strichartz, and the chain rule, we find that

$$
\begin{aligned}
\left\|e^{\frac{i\left(t-t_{j}\right) \Delta}{2}} w\left(t_{j}\right)\right\|_{X\left(I_{j}\right)} & \leq\|w\|_{X\left(I_{j}\right)}+c_{d}\left\|\nabla\left(|w|^{\frac{4}{d-2}} w\right)\right\|_{L_{t}^{2} L_{x}^{\frac{2 d}{d+2}}\left(I_{j}\right)} \\
& \leq \eta+c_{d}\|w\|_{X\left(I_{j}\right)}^{\frac{d+2}{d-2}} \\
& \leq \eta+c_{d} \eta^{\frac{d+2}{d-2}} .
\end{aligned}
$$

Taking $\eta$ sufficiently small relative to $c_{d}$, we obtain (8.10).

Now, choosing $\varepsilon<\eta$ in (8.9) (and adjusting $T$ accordingly), we use the Duhamel formula (8.3), Strichartz, Hölder, and (8.10) to obtain

$$
\begin{aligned}
\|u\|_{X\left(I_{0}\right)} & \leq\left\|e^{\frac{i t \Delta}{2}} u(0)\right\|_{X\left(I_{0}\right)}+c_{d}\|u\|_{X\left(I_{0}\right)}^{\frac{d+2}{d-2}}+C\|V u\|_{L_{t}^{1} H_{x}^{1}\left(I_{0}\right)} \\
& \leq 2 \eta+c_{d}\|u\|_{X\left(I_{0}\right)}^{\frac{d+2}{d-2}}+C_{V} T\|u\|_{L_{t}^{\infty} H_{x}^{1}\left(I_{0}\right)} \\
& \leq 3 \eta+c_{d}\|u\|_{X\left(I_{0}\right)}^{\frac{d+2}{d-2}}
\end{aligned}
$$

By a continuity argument,

$$
\|u\|_{X\left(I_{0}\right)} \leq 4 \eta .
$$

Choosing $\varepsilon$ sufficiently small in (8.9) so that the smallness condition (8.5) is satisfied, we apply Lemma 8.3 with $\|u(0)-w(0)\|_{\dot{H}^{1}}=0$ to find that

$$
\|\nabla(u-w)\|_{S\left(I_{0}\right)} \leq C(E) \varepsilon^{c}
$$


On the interval $I_{1}$, use (8.10), 8.13), and the usual estimates to obtain

$$
\begin{aligned}
\|u\|_{X\left(I_{1}\right)} & \leq\left\|e^{\frac{i\left(t-t_{1}\right) \Delta}{2}} u\left(t_{1}\right)\right\|_{X\left(I_{1}\right)}+c_{d}\|u\|_{X\left(I_{1}\right)}^{\frac{d+2}{d-2}}+C_{V} T\|u\|_{L_{t}^{\infty} H_{x}^{1}\left(I_{1}\right)} \\
& \leq C(E) \varepsilon^{c}+2 \eta+c\|u\|_{X\left(I_{1}\right)}^{\frac{d+2}{d-2}}+\eta
\end{aligned}
$$

where the $C(E)$ in the last line has absorbed the Strichartz constant $c$; this redefinition of $C(E)$ will cause no trouble because the number of times it will occur depends only on $E, d$, and $V$. By by choosing $\varepsilon$ sufficiently small relative to $\eta$ and using continuity, we find that

$$
\|u\|_{X\left(I_{1}\right)} \leq 4 \eta
$$

As before, by choosing $T$ sufficiently small we obtain

$$
\begin{aligned}
\|\nabla(V u)\|_{\dot{N}^{0}\left(I_{1}\right)} & \leq \varepsilon \\
\left\|e^{\frac{i\left(t-t_{1}\right) \Delta}{2}}\left[u\left(t_{1}\right)-w\left(t_{1}\right)\right]\right\|_{X\left(I_{1}\right)} & \leq C(E) \varepsilon^{c}
\end{aligned}
$$

for any $\varepsilon \leq \varepsilon_{0}(E, L)$. Hence by Lemma 8.3 and possibly after modifying $T$ depending on $E$, we get

$$
\|\nabla(u-w)\|_{S\left(I_{1}\right)} \leq C(E) \varepsilon^{c} .
$$

We emphasize that the parameters $\eta, \varepsilon, T$ are chosen so that each depends only on the preceding parameters and on the fixed quantities $d, E, V$.

After iterating at most $J^{\prime}$ times and summing the bounds over $0 \leq j \leq J^{\prime}-1$, we conclude that for $T$ sufficiently small depending on $E$ and $V$,

$$
\|u\|_{X([0, T])} \leq 4 J^{\prime} \eta \leq C(E) .
$$

This establishes the bound (8.7).

\section{REFERENCES}

[1] Thierry Aubin. Équations différentielles non linéaires et problème de Yamabe concernant la courbure scalaire. J. Math. Pures Appl. (9), 55(3):269-296, 1976.

[2] J. Bourgain. Global wellposedness of defocusing critical nonlinear Schrödinger equation in the radial case. J. Amer. Math. Soc., 12(1):145-171, 1999.

[3] Haïm Brézis and Elliott Lieb. A relation between pointwise convergence of functions and convergence of functionals. Proc. Amer. Math. Soc., 88(3):486-490, 1983.

[4] R. Carles. Remarks on nonlinear Schrödinger equations with harmonic potential. Ann. Henri Poincaré, 3(4):757-772, 2002.

[5] Rémi Carles. Nonlinear Schrödinger equations with repulsive harmonic potential and applications. SIAM J. Math. Anal., 35(4):823-843 (electronic), 2003.

[6] Rémi Carles. Global existence results for nonlinear Schrödinger equations with quadratic potentials. Discrete Contin. Dyn. Syst., 13(2):385-398, 2005.

[7] Rémi Carles. Nonlinear schrödinger equation with time-dependent potential. Commun. Math Sci., 9(4):937-964, 2011.

[8] Thierry Cazenave. Semilinear Schrödinger equations, volume 10 of Courant Lecture Notes in Mathematics. New York University Courant Institute of Mathematical Sciences, New York, 2003.

[9] J. Colliander, M. Keel, G. Staffilani, H. Takaoka, and T. Tao. Global well-posedness and scattering for the energy-critical nonlinear Schrödinger equation in $\mathbb{R}^{3}$. Ann. of Math. (2), 167(3):767-865, 2008.

[10] Gerald B. Folland. Harmonic analysis in phase space, volume 122 of Annals of Mathematics Studies. Princeton University Press, Princeton, NJ, 1989.

[11] Waldemar Hebisch. A multiplier theorem for Schrödinger operators. Colloq. Math., 60/61(2):659-664, 1990. 
[12] Alexandru D. Ionescu and Benoit Pausader. The energy-critical defocusing NLS on $\mathbb{T}^{3}$. Duke Math. J., 161(8):1581-1612, 2012.

[13] Alexandru D. Ionescu and Benoit Pausader. Global well-posedness of the energy-critical defocusing NLS on $\mathbb{R} \times \mathbb{T}^{3}$. Comm. Math. Phys., 312(3):781-831, 2012.

[14] Alexandru D. Ionescu, Benoit Pausader, and Gigliola Staffilani. On the global well-posedness of energy-critical Schrödinger equations in curved spaces. Anal. PDE, 5(4):705-746, 2012.

[15] Markus Keel and Terence Tao. Endpoint Strichartz estimates. Amer. J. Math., 120(5):955980, 1998.

[16] Carlos E. Kenig and Frank Merle. Global well-posedness, scattering and blow-up for the energy-critical, focusing, non-linear Schrödinger equation in the radial case. Invent. Math., 166(3):645-675, 2006.

[17] Sahbi Keraani. On the defect of compactness for the Strichartz estimates of the Schrödinger equations. J. Differential Equations, 175(2):353-392, 2001.

[18] Sahbi Keraani. On the blow up phenomenon of the critical nonlinear Schrödinger equation. J. Funct. Anal., 235(1):171-192, 2006.

[19] Rowan Killip, Soonsik Kwon, Shuanglin Shao, and Monica Visan. On the mass-critical generalized KdV equation. Discrete Contin. Dyn. Syst., 32(1):191-221, 2012.

[20] Rowan Killip, Betsy Stovall, and Monica Visan. Scattering for the cubic Klein-Gordon equation in two space dimensions. Trans. Amer. Math. Soc., 364(3):1571-1631, 2012.

[21] Rowan Killip and Monica Visan. The focusing energy-critical nonlinear Schrödinger equation in dimensions five and higher. Amer. J. Math., 132(2):361-424, 2010.

[22] Rowan Killip and Monica Vişan. Nonlinear Schrödinger equations at critical regularity. In Evolution equations, volume 17 of Clay Math. Proc., pages 325-437. Amer. Math. Soc., Providence, RI, 2013.

[23] Rowan Killip, Monica Visan, and Xiaoyi Zhang. Energy-critical NLS with quadratic potentials. Comm. Partial Differential Equations, 34(10-12):1531-1565, 2009.

[24] Rowan Killip, Monica Visan, and Xiaoyi Zhang. Quintic nls in the exterior of a strictly convex obstacle. Preprint, 2012.

[25] Yong-Geun Oh. Cauchy problem and Ehrenfest's law of nonlinear Schrödinger equations with potentials. J. Differential Equations, 81(2):255-274, 1989.

[26] E. Ryckman and M. Visan. Global well-posedness and scattering for the defocusing energycritical nonlinear Schrödinger equation in $\mathbb{R}^{1+4}$. Amer. J. Math., 129(1):1-60, 2007.

[27] Giorgio Talenti. Best constant in Sobolev inequality. Ann. Mat. Pura Appl. (4), 110:353-372, 1976.

[28] Terence Tao and Monica Visan. Stability of energy-critical nonlinear Schrödinger equations in high dimensions. Electron. J. Differential Equations, pages No. 118, 28, 2005.

[29] Terence Tao, Monica Visan, and Xiaoyi Zhang. The nonlinear Schrödinger equation with combined power-type nonlinearities. Comm. Partial Differential Equations, 32(7-9):12811343, 2007.

[30] Michael E. Taylor. Tools for PDE, volume 81 of Mathematical Surveys and Monographs. American Mathematical Society, Providence, RI, 2000. Pseudodifferential operators, paradifferential operators, and layer potentials.

[31] Monica Visan. The defocusing energy-critical nonlinear Schrödinger equation in higher dimensions. Duke Math. J., 138(2):281-374, 2007.

[32] Monica Visan. Oberwolfach seminar: Dispersive equations. 2012.

[33] Jian Zhang. Stability of attractive Bose-Einstein condensates. J. Statist. Phys., 101(3-4):731746,2000 . 